\title{
2006
}

\section{Savannah River Site Annual Illness and Injury Surveillance Report}

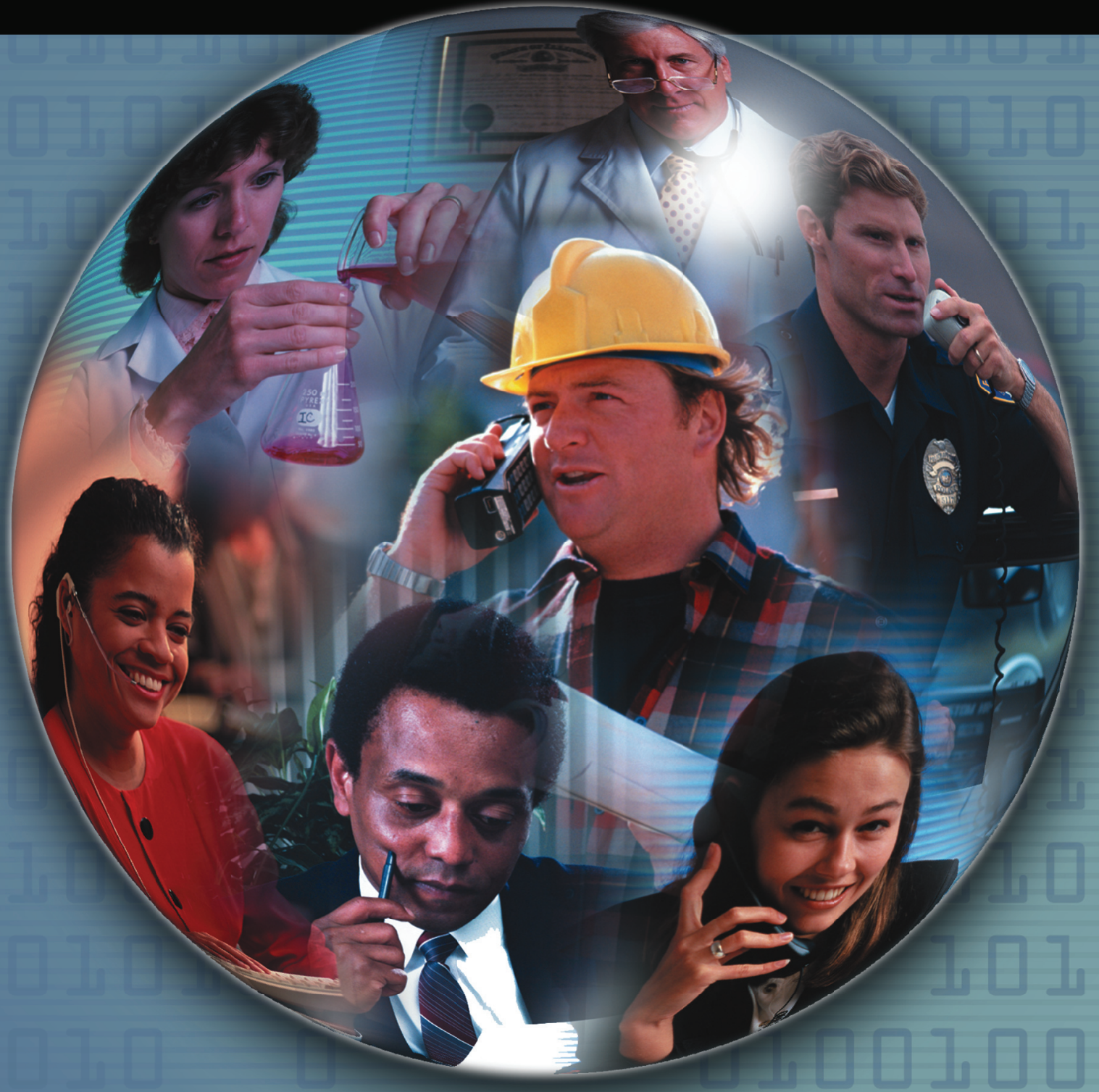




\section{Savannah River Site \\ 2006 Illness and Injury Surveillance Report}

Questions or comments about this report or the Illness and Injury Surveillance Program (IISP) may be directed to:

E-mail:

Dr. Cliff Strader at cliff.strader@hq.doe.gov or Dr. Bonnie Richter at bonnie.richter@hq.doe.gov

or direct letters to:

Mail Stop HS-13 / 270CC

U.S. Department of Energy

1000 Independence Avenue, S.W.

Washington, DC 20585-0270

Additional information about the Department of Energy's Office of Illness and Injury Prevention Programs, the IISP, and annual reports for DOE sites participating in this program can be found at:

\section{http://www.hss.energy.gov/healthsafety/WSHP/epi/surv/}

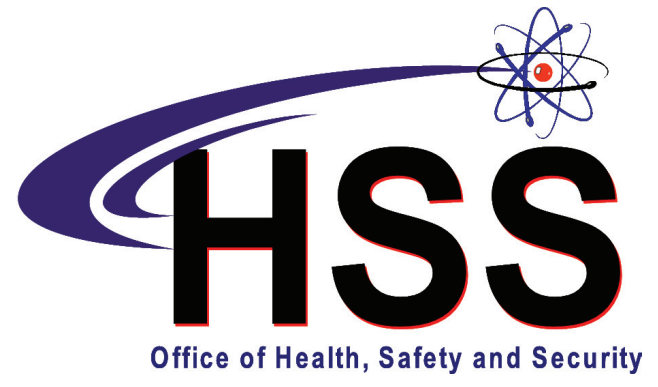

\section{ACKNOWLEDGEMENT}

LifeART images copyright 2000 Lippincott Williams \& Wilkins. All rights reserved.

This document was produced under contract number DE-AC05-06OR23100 between the U.S. Department of Energy and Oak Ridge Associated Universities. 


\section{Savannah River Site 2006 Illness and Injury Surveillance Report}

\section{At A Glance}

The SRS work force has decreased 23 percent since 2003. Over the 4-year period, the ratio of men to women at the site has remained constant while the average age has increased 3 years for men and 2 years for women. In 2006, the SRS work force numbered 9,265 individuals with an average age of 49 years and 47 years among men and women, respectively.

The absence rate has decreased 19.0 percent among women and 8.7 percent among men since 2003, while the length of absence has increased about 2 days for both men and women. A higher absence rate among women than among men was also observed for all IISP sites as a group from 1995 through 2004. Based on 1,068 absences among women and 1,952 absences among men in 2006 , the absence rates at SRS were 49.0 per 100 women and 27.5 per 100 men. The average length of absence was 16 days for women and 15 days for men.

Women in the work force lost 16,859 calendar days due to illness and injury. Respiratory conditions (23 percent), unspecified symptoms (18 percent), and musculoskeletal conditions (17 percent) accounted for 58 percent of all reported diagnoses.

Men lost 29,436 calendar days due to illness and injury. Over half of their reported diagnoses involved respiratory conditions (21 percent), musculoskeletal conditions (18 percent), and unspecified symptoms (16 percent).

Service workers had the highest absence rate among women-Line Operators the highest rate among men. The lowest absence rate among women was observed in the Security and Fire group and among men in the Crafts group. The high rates among Service workers and Line Operators were typical of the pattern noted among all IISP sites as a group from 1995 through 2004. The low rates observed among Security and Fire workers and Crafts workers were not typical of IISP sites as a group during this period.

The number of sentinel health events reported by SRS has decreased 28 percent since 2003. In 2006, SRS workers reported 57 sentinel health event diagnoses. Carpal tunnel syndrome accounted for 42 of these diagnoses.

SRS reported 50 OSHA events in 2006. The rate of reported OSHA events has decreased 49 percent among women and 11 percent among men at SRS, reflecting a broader trend toward decreasing OSHA event rates at IISP sites as a group. The 10 events among women resulted in 35 workdays of restricted activity but no lost days, while the 40 events among men resulted in 412 lost or restricted workdays. 
The type of OSHA accident most frequently reported by men was overexertion and strenuous movements; women most frequently reported falls.

Line operators had the highest rate of OSHA events among women (33 events per 1,000 workers), and Service workers had the highest rates among men (17 events per 1,000 workers). 
The Savannah River Site Work Force

$-2006$

The Work Force by Gender and Age ...........1

The Work Force by Gender and Job

Category ........................................ 1

\section{Number and Length of Absences}

Absence Rate by Gender and Age .............. 2

Number of Days Absent by

Gender and Age

Absence Rate by Job Category

and Gender.

Average Duration of Absence by

Job Category and Gender.

\section{Diagnostic Categories}

Number of Diagnoses and Lost Calendar

Days by Diagnostic Category

(Categorized by ICD-9-CM) and Gender......4

Common Diagnoses Among Female

Workers in 2006 .5

Common Diagnoses Among Male

Workers in 2006

Number of Most Frequently Reported

Diagnoses by Job Category and Gender ....

\section{Rates of Disease Occurrence}

Rates for All Illnesses and Injuries

Combined by Job Category, Gender,

and Age

Rates for Selected Diagnostic Categories

by Job Category, Gender, and Age. .8

\section{Time Trends}

Age-Adjusted Rates for All Diagnoses Combined Among Women and Men from 2003 to 2006
Age-Adjusted Rates for Selected Diagnostic Categories Among Women and Men from

2003 to 2006

Age-Adjusted Rates for All Diagnoses

Combined Among Women and Men by Job

Category from 2003 to $2006 . . . \ldots \ldots \ldots \ldots \ldots . . . . .12$

\section{Sentinel Health Events for Occupations (SHEOs)}

Characteristics of SHEOs by Gender.... 13

SHEO Diagnoses by Gender.... 13

Occupational Safety and Health Administration (OSHA)-Recordable Events

OSHA-Recordable Events by Gender

and Age 14

OSHA-Recordable Events by Job

Category and Gender 14

Diagnostic and Accident Categories for OSHA-Recordable Events

OSHA-Recordable Diagnoses by

Diagnostic Category and Gender 15

OSHA-Recordable Accidents by Type

and Gender. 15

\section{Rates of OSHA-Recordable Events}

OSHA-Recordable Rates by Age and

Job Categories Among Women, All

Diagnoses Combined 16

OSHA-Recordable Rates by Age and

Job Categories Among Men, All

Diagnoses Combined 16

\section{Time Trends for OSHA-Recordable Events}

Age-Adjusted Rates for All OSHA-Recordable Diagnoses Combined Among Women and Men by Job Category from 2003 to $2006 . .17$

\section{Appendices}

Appendices A-W. 19 
The Savannah River Site Work Force - 2006

Figure 1. The Work Force by Gender and Age

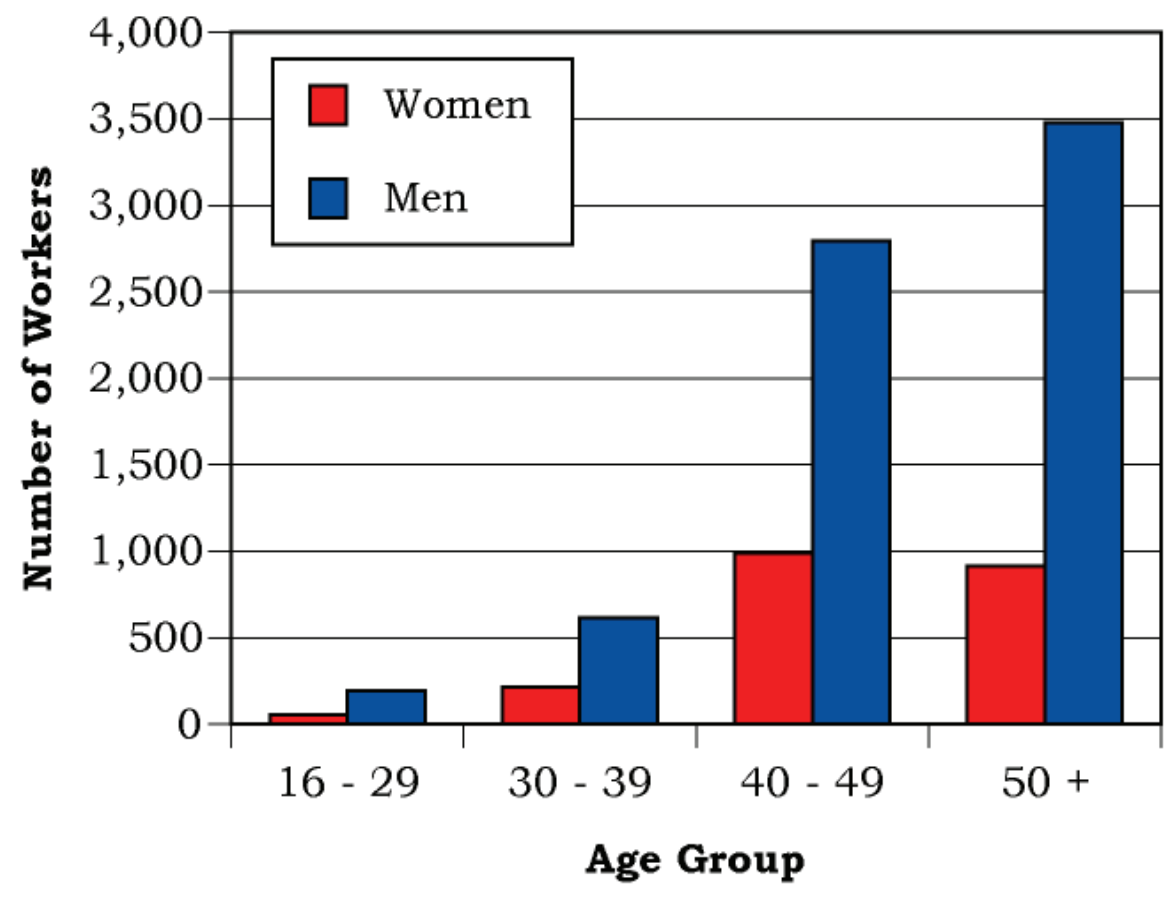

Figure 2. The Work Force by Gender and Job Category

\begin{tabular}{|l|c|c|}
\hline \multirow{2}{*}{ Job Category } & Women & Men \\
\hline \multirow{2}{*}{ Professional } & $\mathbf{1 , 2 1 8}$ & $\mathbf{3 , 4 5 0}$ \\
& $\mathbf{5 6} \%$ & $\mathbf{4 9} \%$ \\
\hline \multirow{2}{*}{ Administrative Support } & $\mathbf{8 3}$ & $\mathbf{4 3}$ \\
& $\mathbf{4} \%$ & $\mathbf{1} \%$ \\
\hline \multirow{2}{*}{ Technical Support } & $\mathbf{5 1 9}$ & $\mathbf{1 , 5 6 6}$ \\
& $\mathbf{2 4} \%$ & $\mathbf{2 2} \%$ \\
\hline \multirow{2}{*}{ Service } & $\mathbf{3 2}$ & $\mathbf{5 9 0}$ \\
& $\mathbf{1} \%$ & $\mathbf{8} \%$ \\
\hline \multirow{2}{*}{ Security and Fire } & $\mathbf{1 2}$ & $\mathbf{1 4 5}$ \\
& $\mathbf{1} \%$ & $\mathbf{2} \%$ \\
\hline \multirow{2}{*}{ Crafts } & $\mathbf{7 5}$ & $\mathbf{5 9 4}$ \\
& $\mathbf{3} \%$ & $\mathbf{8} \%$ \\
\hline \multirow{2}{*}{ Line Operators } & $\mathbf{2 4 0}$ & $\mathbf{6 9 8}$ \\
& $\mathbf{1 1} \%$ & $\mathbf{1 0} \%$ \\
\hline \multirow{2}{*}{ Total } & $\mathbf{2 , 1 7 9}$ & $\mathbf{7 , 0 8 6}$ \\
\hline
\end{tabular}


Number and Length of Absences

Figure 3. Absence Rate by Gender and Age

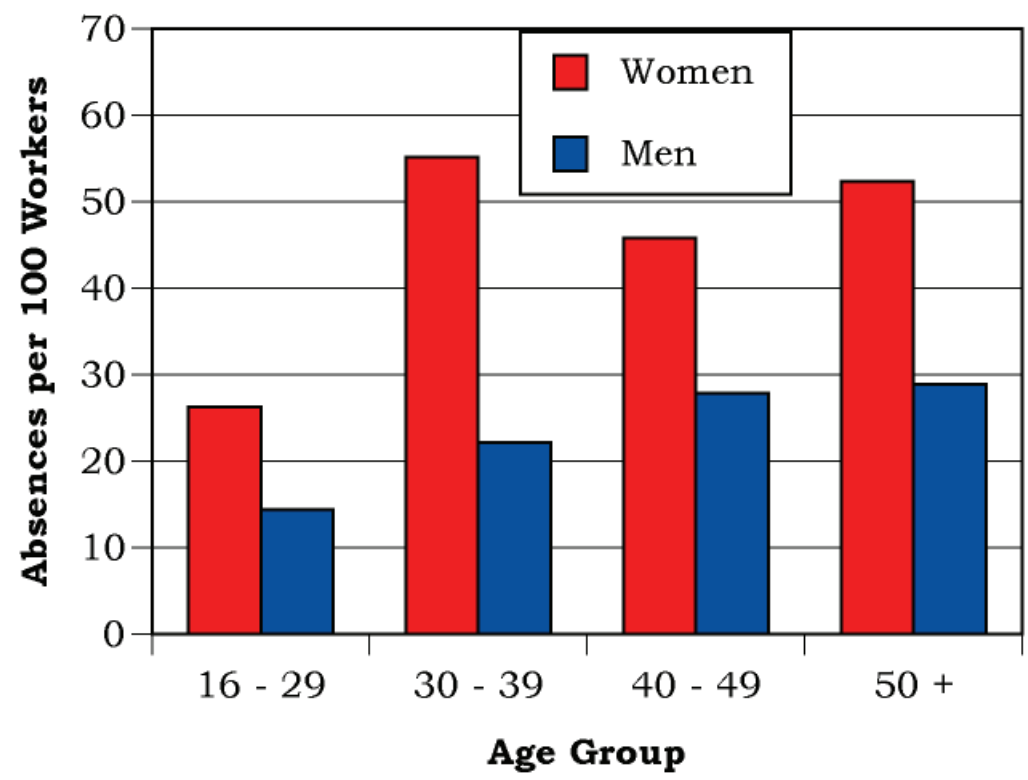

Figure 4. Number of Days Absent by Gender and Age

\begin{tabular}{|c|c|c|c|c|}
\hline \multirow{3}{*}{ Gender } & \multirow{2}{*}{ Age } & Number of & \multicolumn{2}{|c|}{ Number of Days Absent } \\
\cline { 3 - 5 } & & Absences & Total & Average \\
\hline \multirow{4}{*}{ Women } & $16-29$ & 15 & 93 & 6 \\
\cline { 2 - 5 } & $30-39$ & 118 & 1,544 & 13 \\
\cline { 2 - 5 } & $40-49$ & 455 & 7,805 & 17 \\
\cline { 2 - 5 } & $50+$ & 480 & 7,417 & 15 \\
\cline { 2 - 5 } & Total & 1,068 & 16,859 & 16 \\
\hline \multirow{4}{*}{ Men } & $16-29$ & 28 & 268 & 10 \\
\cline { 2 - 5 } & $30-39$ & 137 & 1,400 & 10 \\
\cline { 2 - 5 } & $40-49$ & 780 & 10,927 & 14 \\
\cline { 2 - 5 } & $50+$ & 1,007 & 16,841 & 17 \\
\cline { 2 - 5 } & Total & 1,952 & 29,436 & 15 \\
\hline
\end{tabular}


Figure 5. Absence Rate by Job Category and Gender

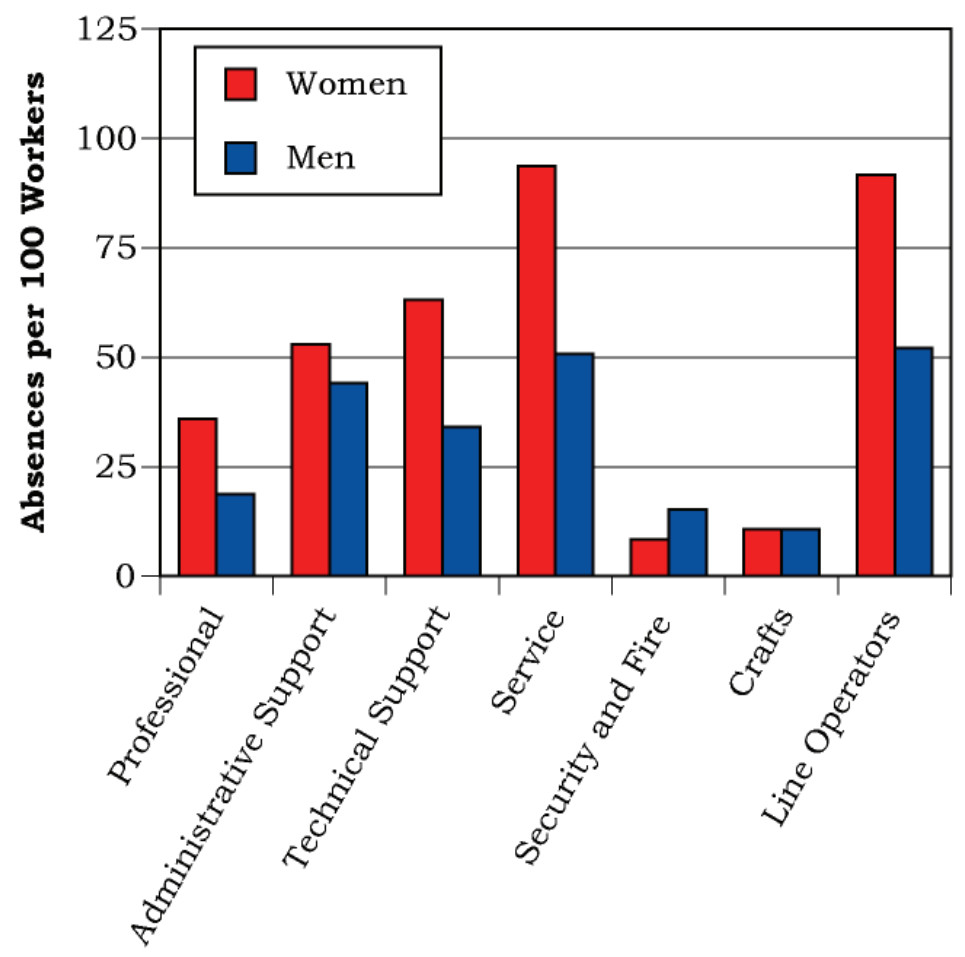

Job Category

Figure 6. Average Duration of Absence by Job Category and Gender

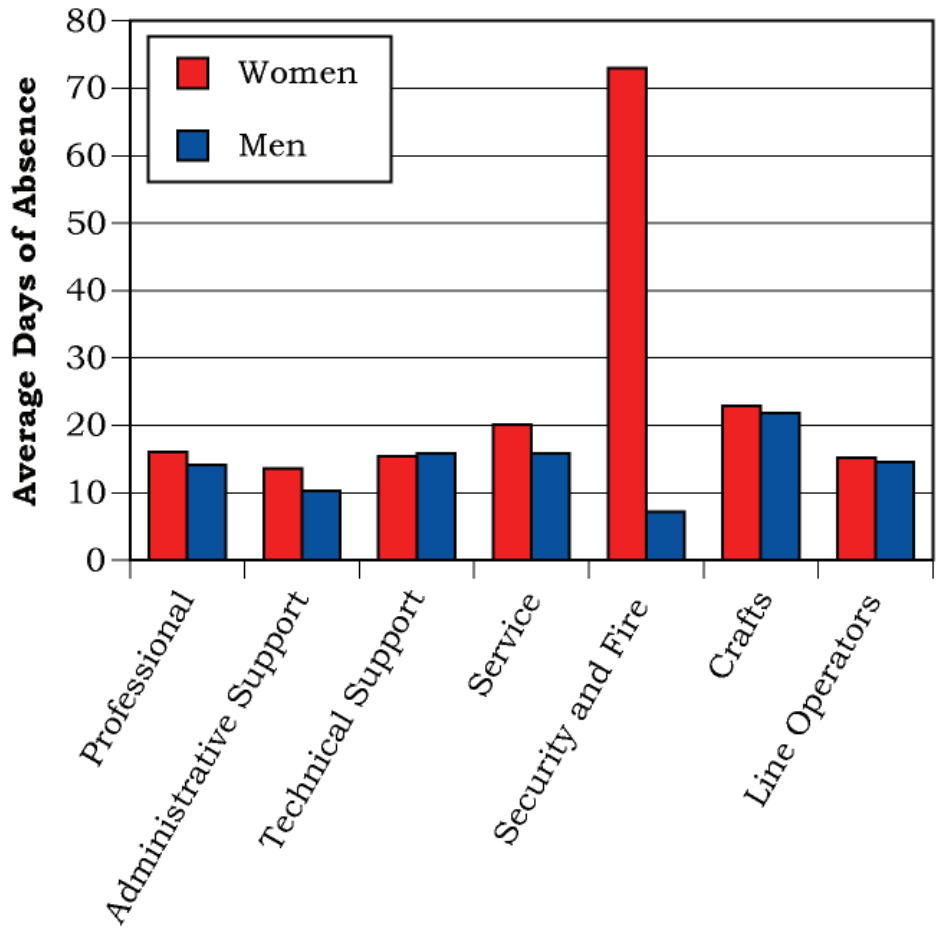

Job Category 


\section{Diagnostic Categories}

\section{Figure 7. Number of Diagnoses and Lost Calendar Days by Diagnostic Category (Categorized by ICD-9-CM) and Gender}

\begin{tabular}{|c|c|c|c|c|}
\hline \multirow[b]{2}{*}{ Diagnostic Category } & \multicolumn{2}{|c|}{ Women } & \multicolumn{2}{|c|}{ Men } \\
\hline & $\begin{array}{l}\text { Number of } \\
\text { Diagnoses }\end{array}$ & $\begin{array}{c}\text { Number } \\
\text { of Lost } \\
\text { Calendar } \\
\text { Days }\end{array}$ & $\begin{array}{l}\text { Number of } \\
\text { Diagnoses }\end{array}$ & $\begin{array}{c}\text { Number } \\
\text { of Lost } \\
\text { Calendar } \\
\text { Days }\end{array}$ \\
\hline Benign Growths & 45 & 1,581 & 22 & 487 \\
\hline Blood & 12 & 198 & 17 & 345 \\
\hline Cancer & 17 & 236 & 59 & 1,823 \\
\hline Digestive & 106 & 1,636 & 223 & 3,487 \\
\hline Endocrine/Metabolic & 42 & 639 & 59 & 1,978 \\
\hline Existing Birth Condition & 1 & 10 & 3 & 14 \\
\hline Genitourinary & 137 & 2,680 & 93 & 1,051 \\
\hline Heart/Circulatory & 59 & 1,136 & 202 & 4,699 \\
\hline Infections/Parasites & 60 & 523 & 86 & 797 \\
\hline Injury & 100 & 1,847 & 276 & 4,894 \\
\hline Miscarriage & 9 & 178 & NA & NA \\
\hline Musculoskeletal & 289 & 4,400 & 515 & 6,800 \\
\hline Nervous System & 99 & 1,638 & 155 & 3,101 \\
\hline Psychological & 26 & 1,031 & 47 & 1,271 \\
\hline Respiratory & 388 & 3,818 & 610 & 5,163 \\
\hline Skin & 17 & 249 & 35 & 612 \\
\hline Unspecified Symptoms & 303 & 2,136 & 464 & 4,914 \\
\hline
\end{tabular}

Note: Lost calendar days for each absence are counted more than once when multiple diagnoses occur in different diagnostic categories for the same absence. 
Figure 8. Common Diagnoses Among Female Workers in 2006

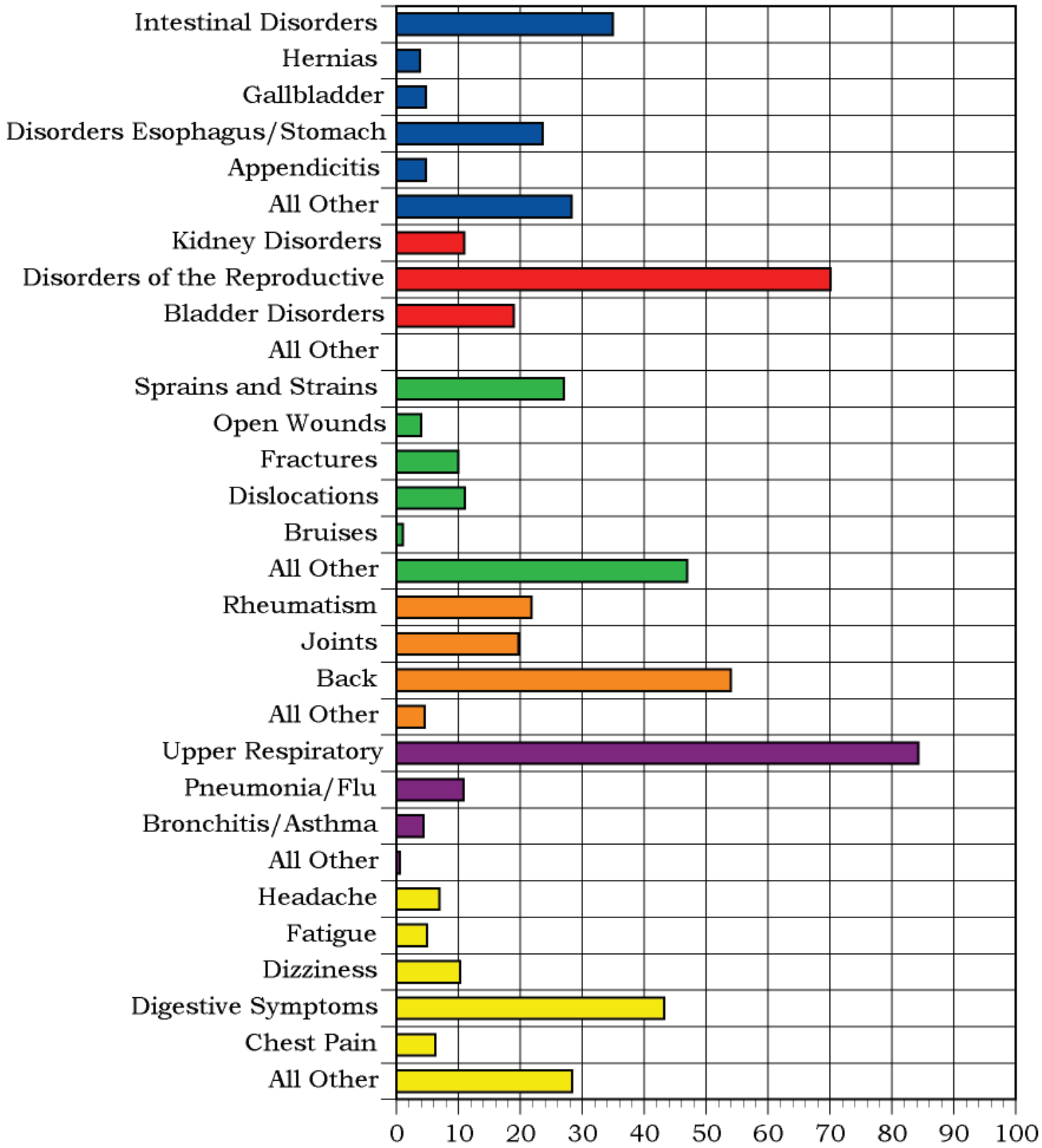

Percent Distribution of Diagnoses Within Diagnostic Category

Digestive, 106 Diagnoses

Genitourinary, 137 Diagnoses

Injury, 100 Diagnoses
Musculoskeletal, 289 Diagnoses

Respiratory, 388 Diagnoses

Unspecified Symptoms, 303 Diagnoses 
Figure 9. Common Diagnoses Among Male Workers in 2006

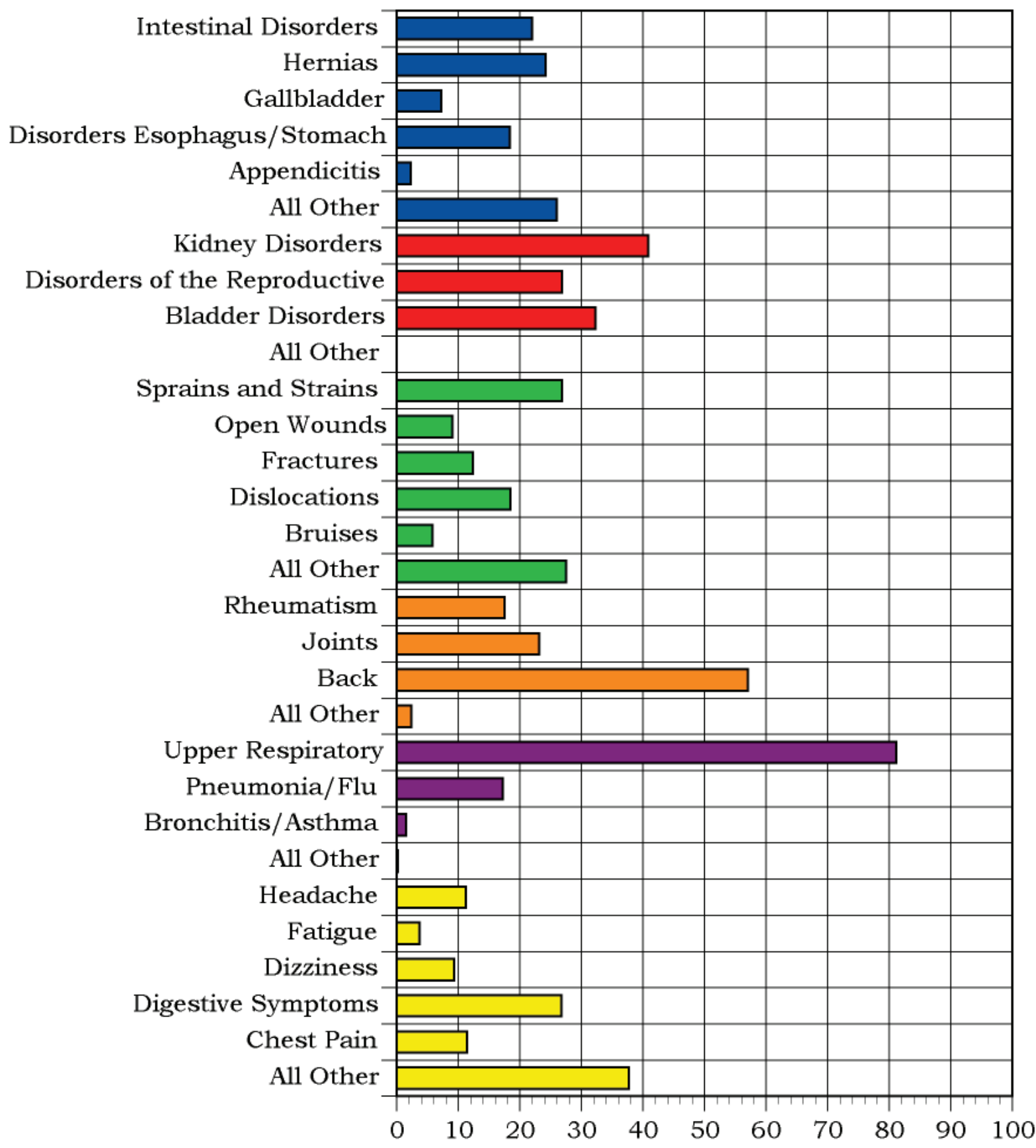

Percent Distribution of Diagnoses Within Diagnostic Category

$\square$ Digestive, 223 Diagnoses

Genitourinary, 93 Diagnoses

Injury, 276 Diagnoses
Musculoskeletal, 515 Diagnoses

Respiratory, 610 Diagnoses

Unspecified Symptoms, 464 Diagnoses 


\section{Figure 10. Number of Most Frequently Reported Diagnoses by Job Category and Gender}

\begin{tabular}{|c|c|c|c|c|}
\hline Job Category & \multicolumn{2}{|l|}{ Men } & \multicolumn{2}{|l|}{ Women } \\
\hline \multirow{3}{*}{ Professional } & Respiratory & 171 & Respiratory & 137 \\
\hline & Musculoskeletal & 166 & Musculoskeletal & 105 \\
\hline & Unspecified Symptoms & 135 & Unspecified Symptoms & 79 \\
\hline \multirow{4}{*}{ Administrative Support } & Respiratory & 7 & Unspecified Symptoms & 20 \\
\hline & Musculoskeletal & 6 & Respiratory & 15 \\
\hline & Genitourinary & 3 & Nervous System & 12 \\
\hline & Unspecified Symptoms & 3 & & \\
\hline \multirow{3}{*}{ Technical Support } & Respiratory & 187 & Unspecified Symptoms & 129 \\
\hline & Musculoskeletal & 160 & Respiratory & 126 \\
\hline & Unspecified Symptoms & 137 & Musculoskeletal & 94 \\
\hline \multirow{3}{*}{ Service } & Respiratory & 104 & Respiratory & 19 \\
\hline & Unspecified Symptoms & 79 & Unspecified Symptoms & 11 \\
\hline & Musculoskeletal & 73 & Musculoskeletal & 6 \\
\hline \multirow{4}{*}{ Security and Fire } & Respiratory & 5 & Benign Growths & 1 \\
\hline & Unspecified Symptoms & 5 & & \\
\hline & Heart/Circulatory & 4 & & \\
\hline & Musculoskeletal & 4 & & \\
\hline \multirow{3}{*}{ Crafts } & Injury & 15 & Musculoskeletal & 2 \\
\hline & Musculoskeletal & 12 & Respiratory & 2 \\
\hline & Respiratory & 12 & Unspecified Symptoms & 2 \\
\hline \multirow{3}{*}{ Line Operators } & Respiratory & 124 & Respiratory & 89 \\
\hline & Unspecified Symptoms & 101 & Musculoskeletal & 73 \\
\hline & Musculoskeletal & 94 & Unspecified Symptoms & 62 \\
\hline
\end{tabular}




\section{Rates of Disease Occurrence}

Figure 11. Rates for All Illnesses and Injuries Combined by Job Category, Gender, and Age

\begin{tabular}{|c|c|c|c|c|}
\hline \multirow{2}{*}{$\begin{array}{c}\text { All Illnesses \& } \\
\text { Injuries Combined }\end{array}$} & \multicolumn{4}{|c|}{ Rate per 1,000} \\
\hline & Job Category & Age & Men & Women \\
\hline & \multirow{2}{*}{ Professional } & $<50$ & 227 & 432 \\
\hline & & $50+$ & 323 & 641 \\
\hline & \multirow{2}{*}{ Administrative Support } & $<50$ & 1,118 & 1,167 \\
\hline & & $50+$ & 269 & 617 \\
\hline & \multirow{2}{*}{ Technical Support } & $<50$ & 493 & 1,224 \\
\hline & & $50+$ & 526 & 949 \\
\hline & \multirow{2}{*}{ Service } & $<50$ & 728 & 1,000 \\
\hline & & $50+$ & 812 & 2,600 \\
\hline & \multirow{2}{*}{ Security and Fire } & $<50$ & 95 & 0 \\
\hline & & $50+$ & 296 & 500 \\
\hline & \multirow{2}{*}{ Crafts } & $<50$ & 97 & 174 \\
\hline & & $50+$ & 201 & 103 \\
\hline & \multirow{2}{*}{ Line Operators } & $<50$ & 841 & 1,420 \\
\hline 5 & & $50+$ & 642 & 1,633 \\
\hline
\end{tabular}

Figure 12. Rates for Selected Diagnostic Categories by Job Category, Gender, and Age

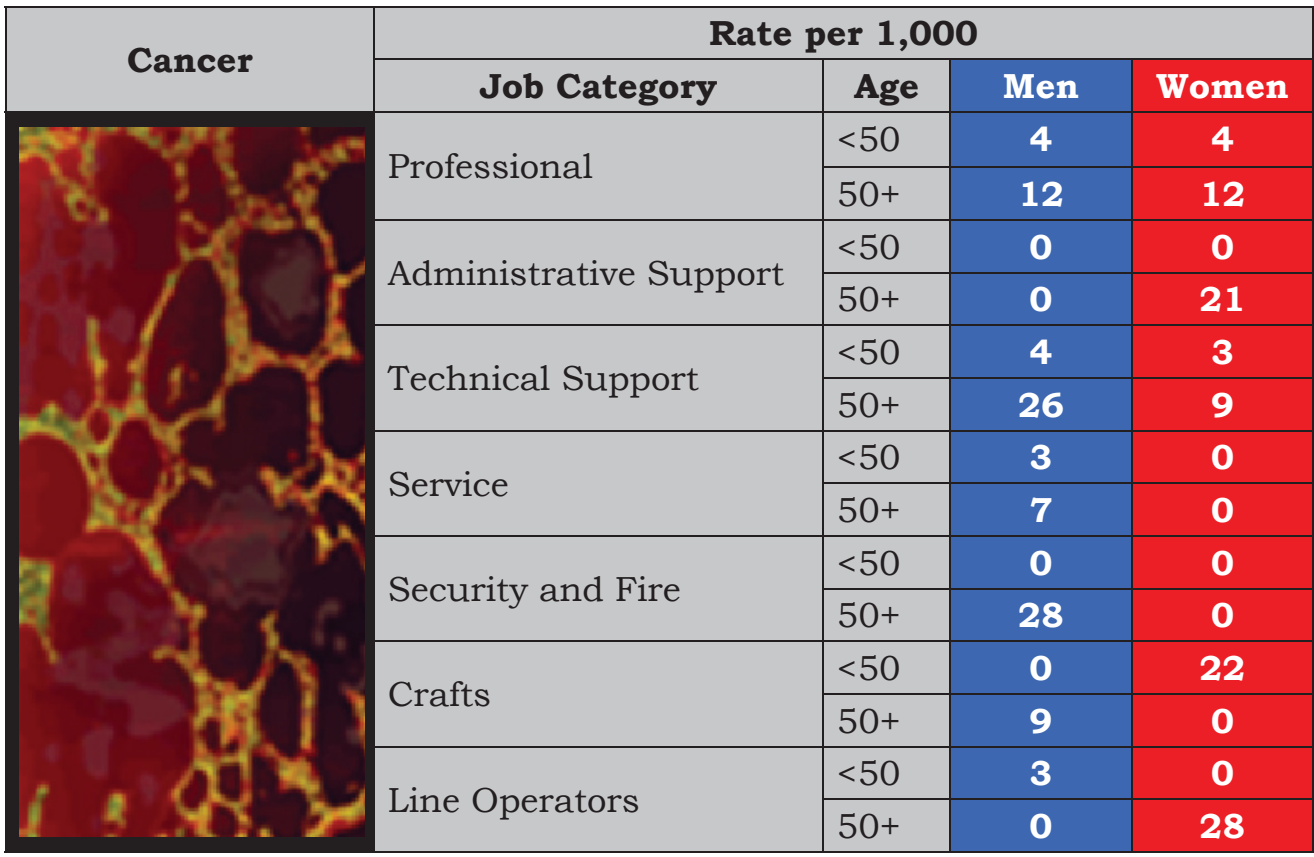




\section{Figure 12. Rates for Selected Diagnostic Categories by Job Category, Gender, and Age (Continued)}

\begin{tabular}{|c|c|c|c|c|}
\hline \multirow{2}{*}{ Heart/Circulatory } & \multicolumn{4}{|c|}{ Rate per 1,000} \\
\hline & Job Category & Age & Men & Women \\
\hline \multirow{14}{*}{$\mathrm{W}$} & \multirow{2}{*}{ Professional } & $<50$ & 11 & 21 \\
\hline & & $50+$ & 26 & 18 \\
\hline & \multirow{2}{*}{ Administrative Support } & $<50$ & 59 & 0 \\
\hline & & $50+$ & 38 & 21 \\
\hline & \multirow{2}{*}{ Technical Support } & $<50$ & 31 & 50 \\
\hline & & $50+$ & 51 & 32 \\
\hline & \multirow{2}{*}{ Service } & $<50$ & 67 & 0 \\
\hline & & $50+$ & 58 & 67 \\
\hline & \multirow{2}{*}{ Security and Fire } & $<50$ & 0 & 0 \\
\hline & & $50+$ & 56 & 0 \\
\hline & \multirow{2}{*}{ Crafts } & $<50$ & 6 & 0 \\
\hline & & $50+$ & 34 & 0 \\
\hline & \multirow{2}{*}{ Line Operators } & $<50$ & 38 & 38 \\
\hline & & $50+$ & 16 & 55 \\
\hline
\end{tabular}

\begin{tabular}{|c|c|c|c|c|}
\hline \multirow{2}{*}{ Respiratory } & \multicolumn{4}{|c|}{ Rate per 1,000} \\
\hline & Job Category & Age & Men & Women \\
\hline & \multirow{2}{*}{ Professional } & $<50$ & 45 & 81 \\
\hline & & $50+$ & 54 & 159 \\
\hline & \multirow{2}{*}{ Administrative Support } & $<50$ & 294 & 222 \\
\hline & & $50+$ & 77 & 149 \\
\hline & \multirow{2}{*}{ Technical Support } & $<50$ & 151 & 261 \\
\hline & & $50+$ & 90 & 218 \\
\hline & \multirow{2}{*}{ Service } & $<50$ & 195 & 353 \\
\hline & & $50+$ & 155 & 867 \\
\hline & \multirow{2}{*}{ Security and Fire } & $<50$ & 27 & 0 \\
\hline & & $50+$ & 42 & 0 \\
\hline & \multirow{2}{*}{ Crafts } & $<50$ & 11 & 43 \\
\hline & & $50+$ & 34 & 0 \\
\hline & \multirow{2}{*}{ Line Operators } & $<50$ & 202 & 351 \\
\hline & & $50+$ & 147 & 394 \\
\hline
\end{tabular}


Figure 12. Rates for Selected Diagnostic Categories by Job Category, Gender, and Age (Continued)

\begin{tabular}{|c|c|c|c|c|}
\hline \multirow{2}{*}{ Injury } & \multicolumn{4}{|c|}{ Rate per 1,000} \\
\hline & Job Category & Age & Men & Women \\
\hline & \multirow{2}{*}{ Professional } & $<50$ & 31 & 29 \\
\hline & & $50+$ & 24 & 14 \\
\hline & \multirow{2}{*}{ Administrative Support } & $<50$ & 0 & 56 \\
\hline & & $50+$ & 0 & 21 \\
\hline & \multirow{2}{*}{ Technical Support } & $<50$ & 37 & 99 \\
\hline & & $50+$ & 39 & 60 \\
\hline & \multirow{2}{*}{ Service } & $<50$ & 45 & 59 \\
\hline & & $50+$ & 105 & 267 \\
\hline & \multirow{2}{*}{ Security and Fire } & $<50$ & 0 & 0 \\
\hline & & $50+$ & 0 & 0 \\
\hline & \multirow{2}{*}{ Crafts } & $<50$ & 22 & 0 \\
\hline & & $50+$ & 30 & 34 \\
\hline & \multirow{2}{*}{ Line Operators } & $<50$ & 102 & 99 \\
\hline & & $50+$ & 78 & 64 \\
\hline
\end{tabular}

\section{Time Trends}

Figure 13. Age-Adjusted Rates for All Diagnoses Combined Among Women and Men from 2003 to 2006*

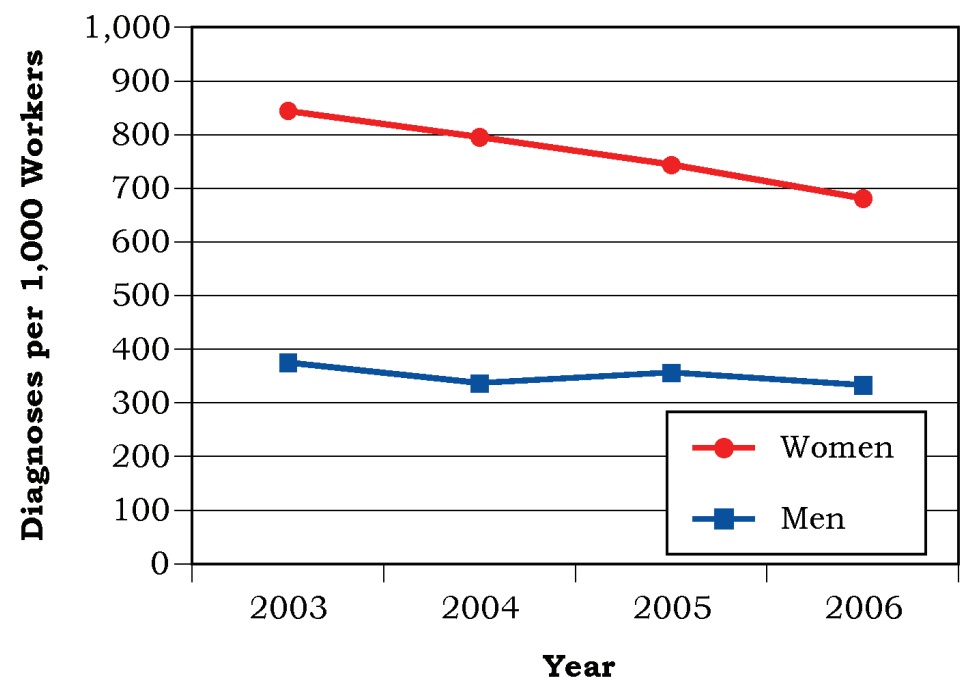

* Standardized to age distribution of 2000 U.S. population. 
Figure 14. Age-Adjusted Rates for Selected Diagnostic Categories Among Women and Men from 2003 to 2006*

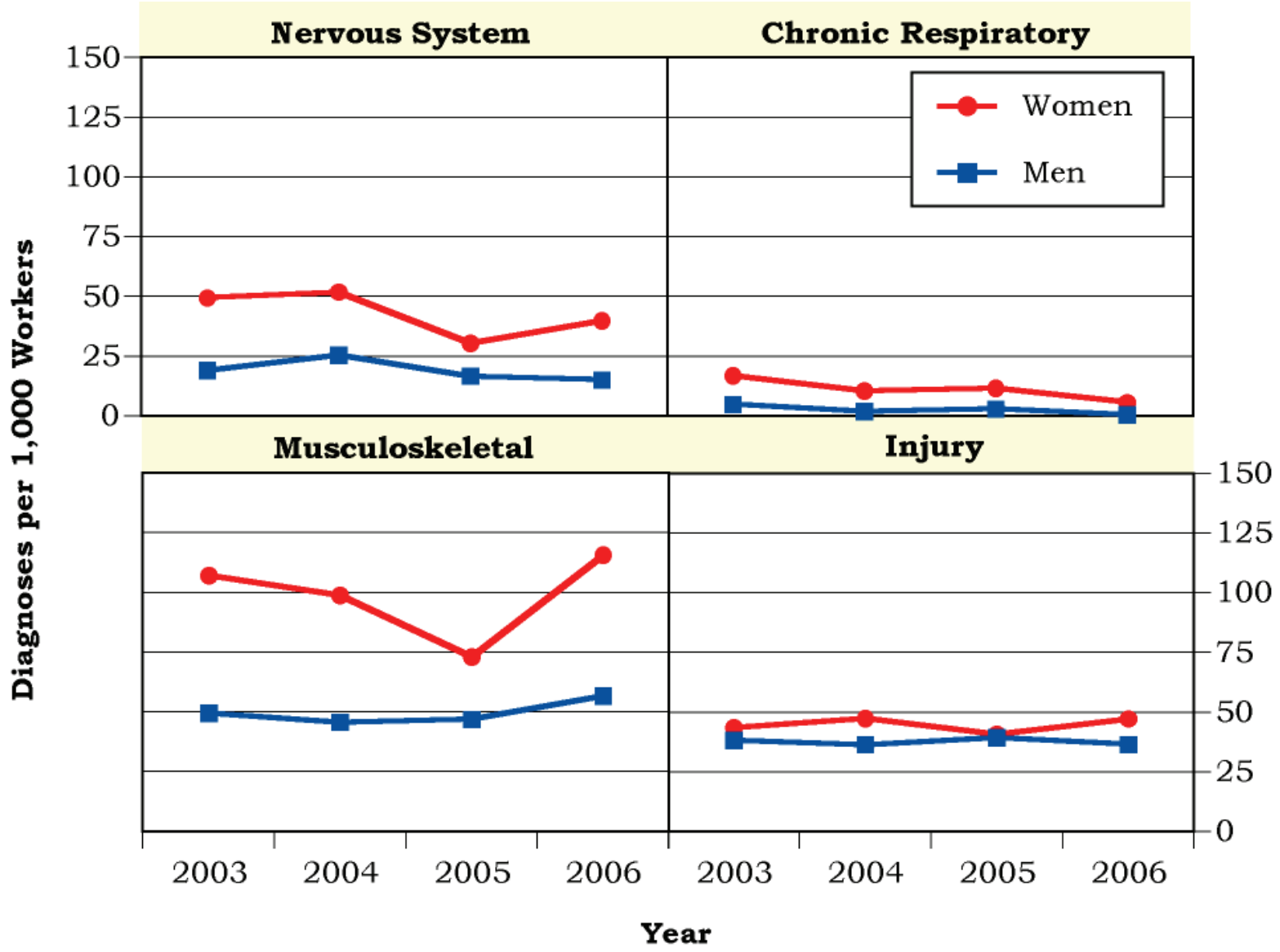

* Standardized to age distribution of 2000 U.S. population. 
Figure 15. Age-Adjusted Rates for All Diagnoses Combined Among Women and Men by Job Category from 2003 to 2006*
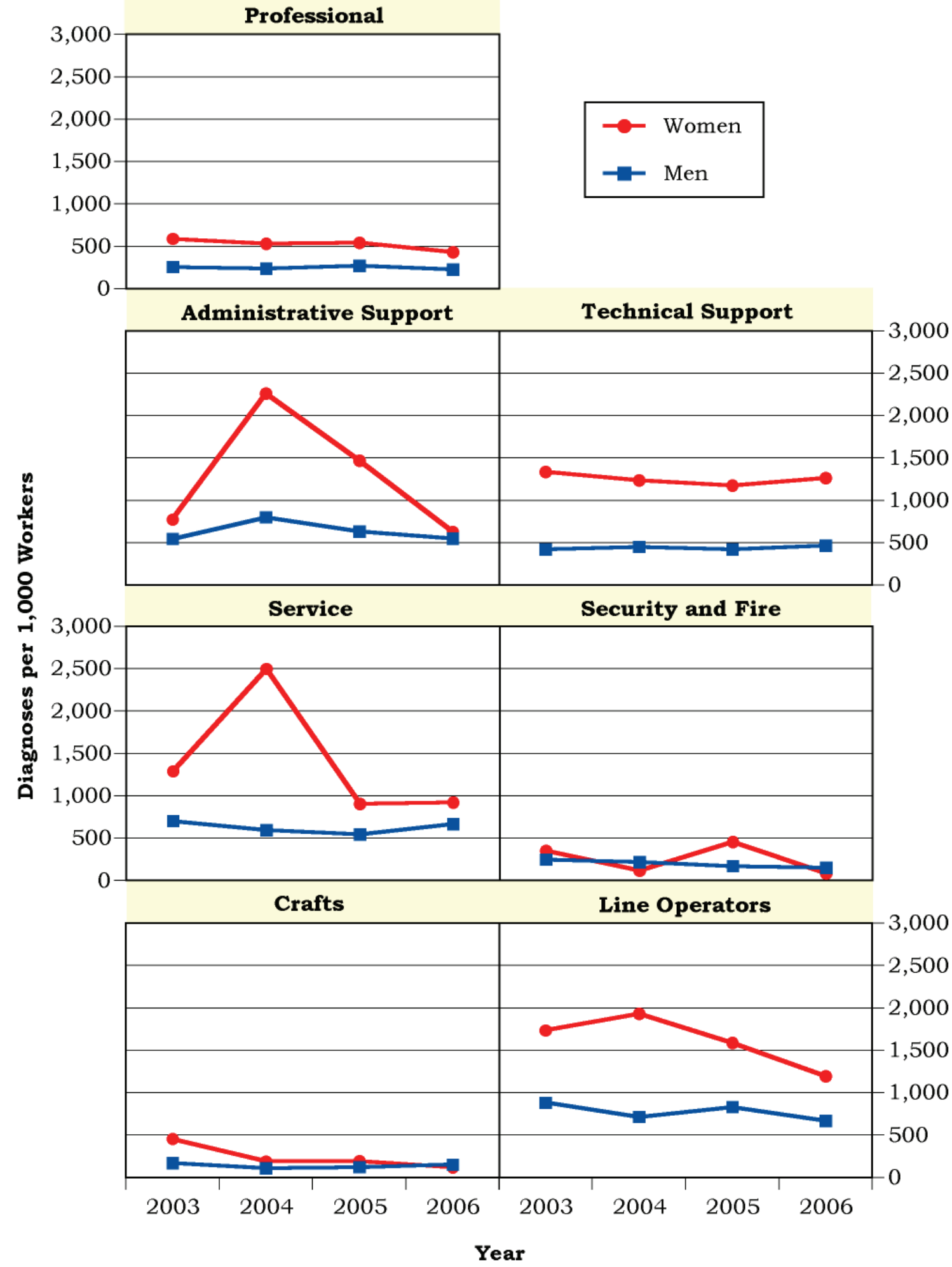

* Standardized to age distribution of 2000 U.S. population. 


\section{Sentinel Health Events for Occupations (SHEOs)}

An occupational sentinel health event (SHEO) is a disease, disability, or death that is likely to be occupationally related. Although sentinel health events may indicate an occupational exposure, many may result from nonoccupational exposures. Sentinel health events are therefore assessed in two categories:

Definite Sentinel Health Events: Diseases that are unlikely to occur in the absence of an occupational exposure (e.g., asbestosis).

Possible Sentinel Health Events: Diseases that may be occupational but can also occur in the absence of an occupational exposure (e.g., lung cancer or carpal tunnel syndrome).

Figure 16. Characteristics of SHEOs by Gender

\begin{tabular}{|l|c|c|c|c|}
\hline \multirow{2}{*}{} & \multicolumn{2}{|c|}{$\begin{array}{c}\text { Total Number of } \\
\text { SHEO Diagnoses }\end{array}$} & \multicolumn{2}{c|}{$\begin{array}{c}\text { Total Number of } \\
\text { Days Absent }\end{array}$} \\
\cline { 2 - 5 } & Men & Women & Men & Women \\
\hline Definite & 5 & 1 & 3 & 818 \\
\hline Possible & 26 & 25 & 434 & 857 \\
\hline Total & 31 & 26 & 437 & 1,675 \\
\hline
\end{tabular}

Figure 17. SHEO Diagnoses by Gender

\begin{tabular}{|l|c|c|}
\hline \multirow{2}{*}{\multicolumn{1}{|c|}{ Diagnoses }} & \multicolumn{2}{c|}{ Gender } \\
\cline { 2 - 3 } & Women & Men \\
\hline Carpal Tunnel Syndrome & 23 & 19 \\
\hline Pneumonconiosis (Berylliosis) & 0 & 1 \\
\hline Musculoskeletal Conditions & 0 & 1 \\
\hline Injuries & 1 & 1 \\
\hline Other Conditions & 2 & 9 \\
\hline
\end{tabular}


Occupational Safety and Health Administration (OSHA)-Recordable Events Figure 18. OSHA-Recordable Events by Gender and Age

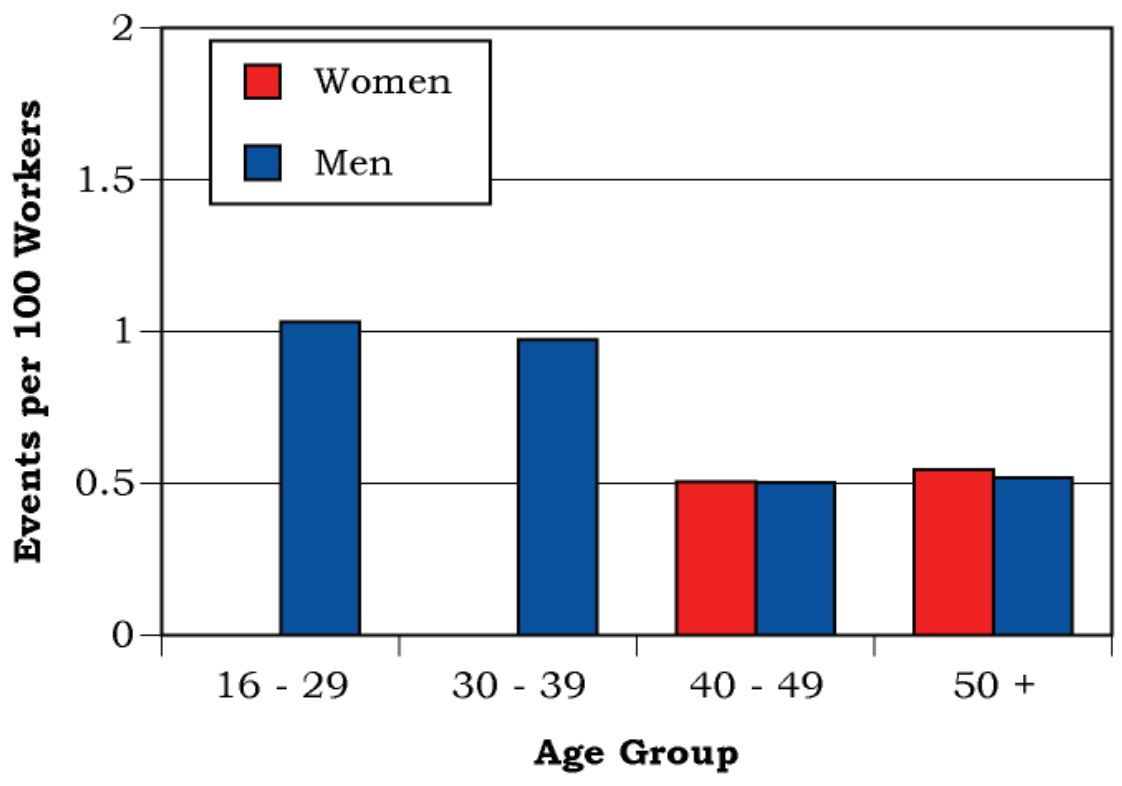

Figure 19. OSHA-Recordable Events by Job Category and Gender

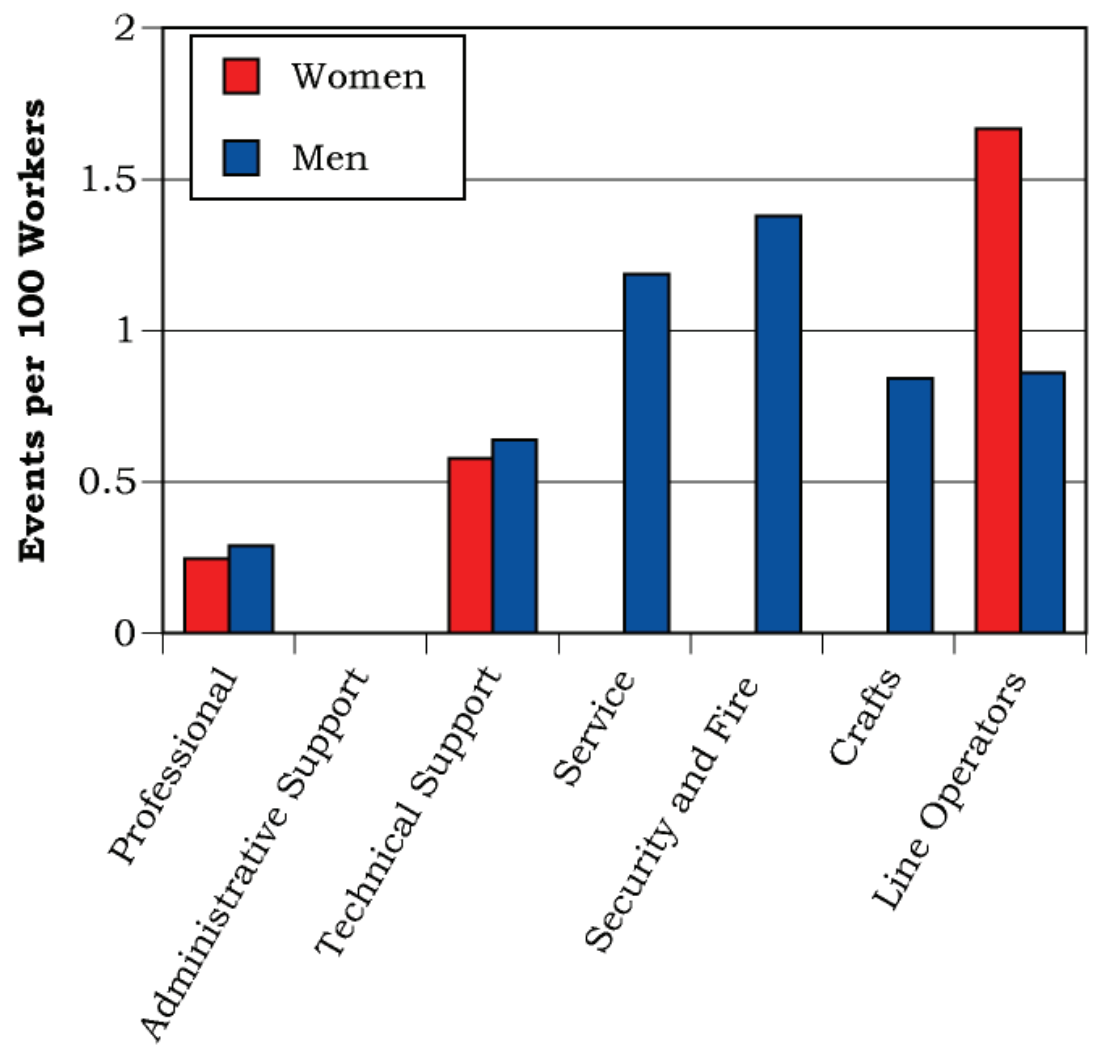

Job Category 


\section{Diagnostic and Accident Categories for OSHA-Recordable Events}

Figure 20. OSHA-Recordable Diagnoses by Diagnostic Category and Gender

\begin{tabular}{|l|c|c|}
\hline \multirow{2}{*}{\multicolumn{1}{|c|}{ Diagnostic Category }} & \multicolumn{2}{c|}{ Gender } \\
\cline { 2 - 3 } & Women & Men \\
\hline Digestive & $\mathbf{1}$ & $\mathbf{2}$ \\
\hline Musculoskeletal & $\mathbf{0}$ & $\mathbf{6}$ \\
\hline Nervous System & $\mathbf{3}$ & $\mathbf{1}$ \\
\hline Respiratory & $\mathbf{3}$ & $\mathbf{5}$ \\
\hline Unspecified Symptoms & $\mathbf{1 0}$ & $\mathbf{3}$ \\
\hline Injury & $\mathbf{1}$ & $\mathbf{0}$ \\
\hline Fractures - Neck, Trunk & $\mathbf{0}$ & $\mathbf{1}$ \\
\hline Fractures - Upper Limb & $\mathbf{0}$ & $\mathbf{2}$ \\
\hline Back Sprains \& Strains & $\mathbf{3}$ & $\mathbf{3}$ \\
\hline Other Sprains \& Strains & $\mathbf{1}$ & $\mathbf{1}$ \\
\hline Open Wounds - Head, Neck, Trunk & $\mathbf{1}$ & $\mathbf{9}$ \\
\hline Open Wounds - Upper Limb & $\mathbf{1}$ & $\mathbf{8}$ \\
\hline Superficial Injuries & $\mathbf{2}$ & $\mathbf{7}$ \\
\hline Bruises & $\mathbf{0}$ & $\mathbf{2}$ \\
\hline Foreign Bodies Entering Orifice & $\mathbf{0}$ & $\mathbf{3}$ \\
\hline Burns & $\mathbf{1}$ & $\mathbf{0}$ \\
\hline Adverse Reactions to External Causes & & \\
\hline
\end{tabular}

Figure 21. OSHA-Recordable Accidents by Type and Gender

\begin{tabular}{|c|c|c|}
\hline \multirow{3}{*}{ Accident Category } & \multicolumn{2}{|c|}{ Gender } \\
\hline & Women & Men \\
\hline & $\begin{array}{l}\text { Number of } \\
\text { Accidents }\end{array}$ & $\begin{array}{l}\text { Number of } \\
\text { Accidents }\end{array}$ \\
\hline Motor Vehicle Traffic & 0 & 1 \\
\hline Falls & 3 & 4 \\
\hline Fire & 2 & 3 \\
\hline Natural/Environmental Factors & 1 & 0 \\
\hline Submersion/Suffocation/Foreign Bodies & 0 & 2 \\
\hline Other Accidents & 3 & 27 \\
\hline Struck by an Object & 0 & 3 \\
\hline Caught Between Objects & 0 & 6 \\
\hline Cutting/Piercing Instrument/Object & 1 & 6 \\
\hline $\begin{array}{l}\text { Hot, Corrosive, or Caustic Material/ } \\
\text { Steam }\end{array}$ & 0 & 1 \\
\hline Overexertion/Strenuous Movements & 2 & 11 \\
\hline Total & 9 & 37 \\
\hline
\end{tabular}




\section{Rates of OSHA-Recordable Events}

Figure 22. OSHA-Recordable Rates by Age and Job Categories Among Women, All Diagnoses Combined

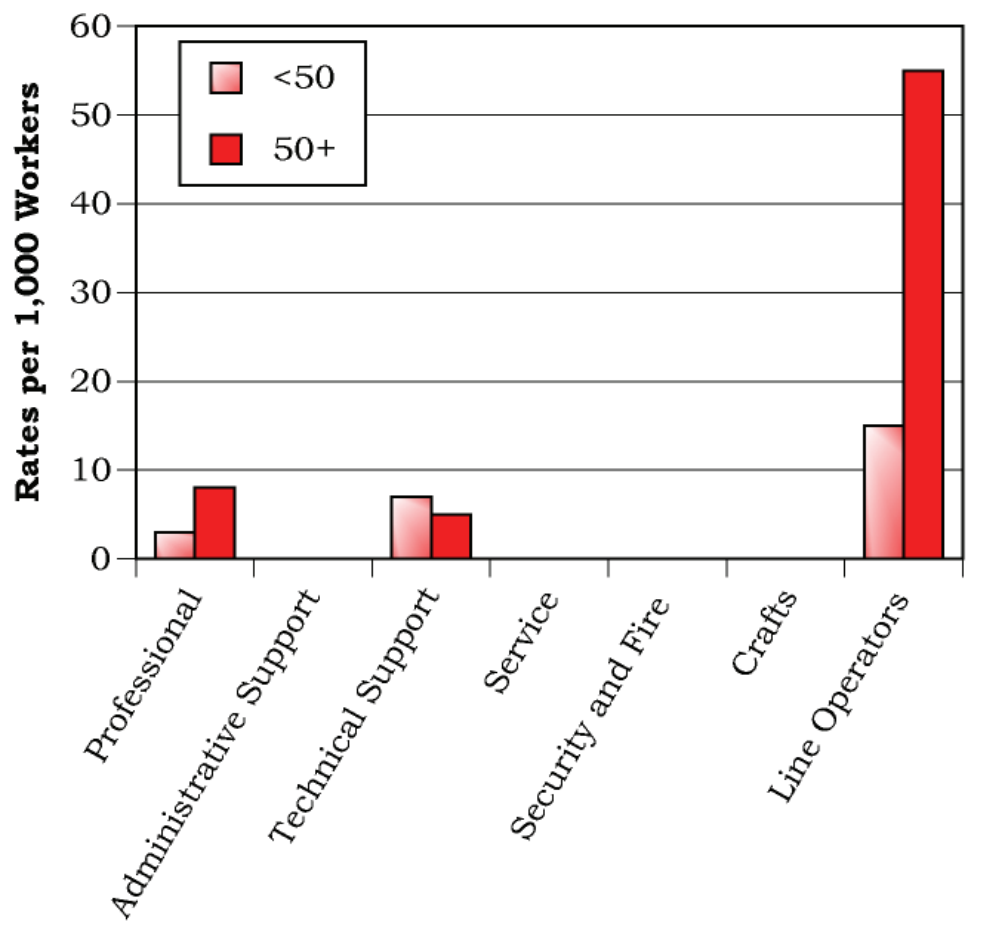

Job Category

Figure 23. OSHA-Recordable Rates by Age and Job Categories Among Men, All Diagnoses Combined

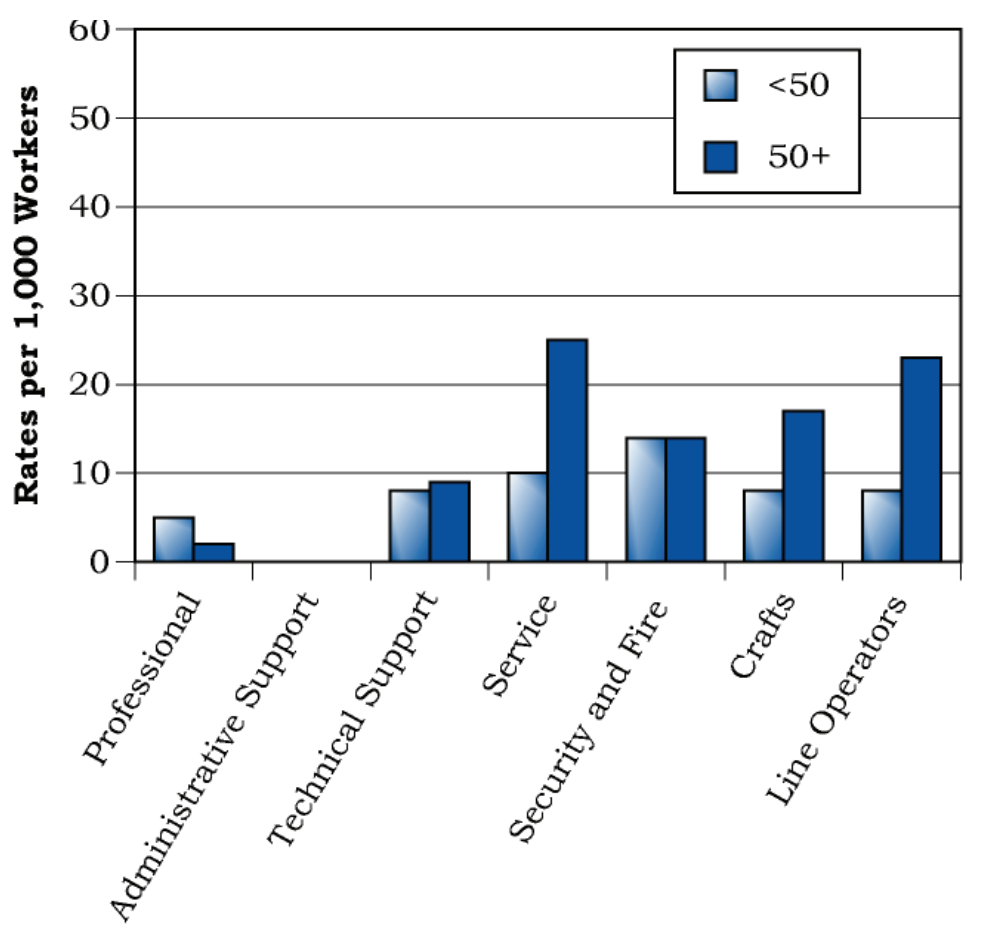

Job Category 


\section{Time Trends for OSHA-Recordable Events}

Figure 24. Age-Adjusted Rates for All OSHA-Recordable Diagnoses Combined Among Women and Men by Job Category from 2003 to 2006*

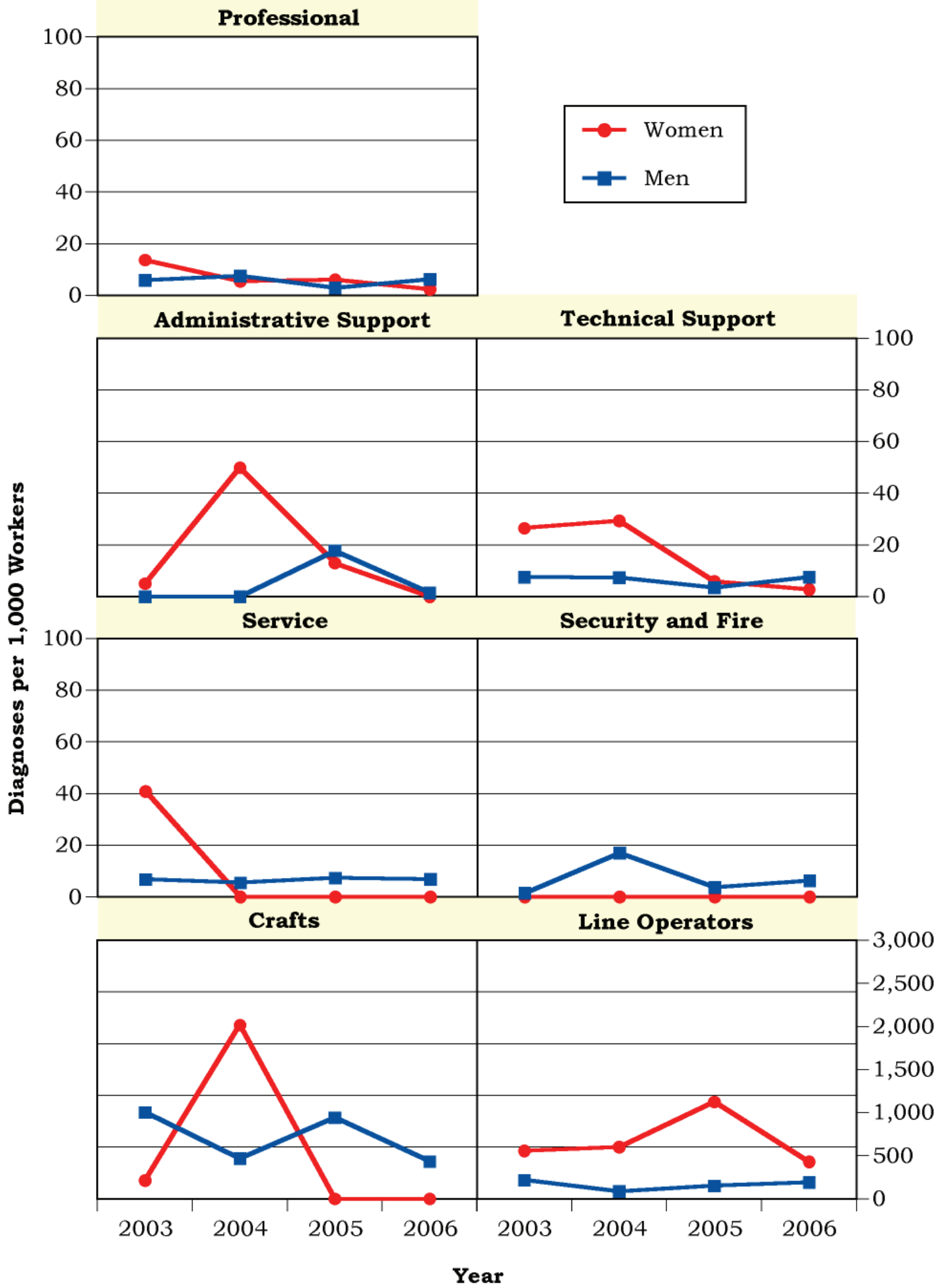

* Standardized to age distribution of 2000 U.S. population. 


\section{Appendices}


Savannah River Site 2006

Absence Data

Appendix A. Work Force by Gender, Age, and Job Category

\begin{tabular}{|c|c|c|c|c|c|c|c|c|c|c|c|}
\hline \multirow{3}{*}{ Job Category } & \multicolumn{5}{|c|}{ Women } & \multicolumn{5}{|c|}{ Men } & \multirow[b]{3}{*}{ TOTAL } \\
\hline & \multicolumn{4}{|c|}{ Age Group } & \multirow[b]{2}{*}{ TOTAL } & \multicolumn{4}{|c|}{ Age Group } & \multirow[b]{2}{*}{ TOTAL } & \\
\hline & $16-29$ & $30-39$ & $40-49$ & $50+$ & & $16-29$ & 30 - 39 & $40-49$ & $50+$ & & \\
\hline Professional & 44 & 127 & 549 & 498 & 1,218 & 123 & 303 & 1,260 & 1,764 & 3,450 & 4,668 \\
\hline Administrative Support & 0 & 8 & 28 & 47 & 83 & 0 & 3 & 14 & 26 & 43 & 126 \\
\hline Technical Support & 6 & 51 & 246 & 216 & 519 & 25 & 105 & 634 & 802 & 1,566 & 2,085 \\
\hline Service & 0 & 0 & 17 & 15 & 32 & 0 & 17 & 296 & 277 & 590 & 622 \\
\hline Security and Fire & 0 & 1 & 9 & 2 & 12 & 0 & 12 & 62 & 71 & 145 & 157 \\
\hline Crafts & 6 & 9 & 31 & 29 & 75 & 37 & 120 & 203 & 234 & 594 & 669 \\
\hline Line Operators & 1 & 18 & 112 & 109 & 240 & 9 & 57 & 325 & 307 & 698 & 938 \\
\hline TOTAL & 57 & 214 & 992 & 916 & 2,179 & 194 & 617 & 2,794 & 3,481 & 7,086 & 9,265 \\
\hline
\end{tabular}

Appendix B. Age Distribution of the Work Force by Gender

\begin{tabular}{|l|r|r|r|r|r|r|r|r|}
\hline \multirow{3}{*}{ Year } & \multicolumn{4}{|c|}{ Women } & \multicolumn{4}{c|}{ Men } \\
\cline { 2 - 9 } & \multicolumn{1}{|c|}{ Percent Distribution by Age Group } & \multicolumn{1}{c|}{ Percent Distribution by Age Group } \\
\cline { 2 - 9 } & $\mathbf{1 6}-\mathbf{2 9}$ & $\mathbf{3 0}-\mathbf{3 9}$ & $\mathbf{4 0}-\mathbf{4 9}$ & $\mathbf{5 0}+$ & $\mathbf{1 6}-\mathbf{2 9}$ & $\mathbf{3 0}-\mathbf{3 9}$ & $\mathbf{4 0}-\mathbf{4 9}$ & $\mathbf{5 0}+$ \\
\hline $\mathbf{1 9 9 4}$ & 23.92 & 41.93 & 26.45 & 7.71 & 16.20 & 37.97 & 31.48 & 14.35 \\
\hline $\mathbf{1 9 9 5}$ & 18.31 & 42.58 & 29.86 & 9.25 & 11.66 & 37.77 & 34.26 & 16.31 \\
\hline $\mathbf{1 9 9 6}$ & 12.75 & 41.43 & 34.53 & 11.29 & 7.89 & 35.53 & 38.82 & 17.76 \\
\hline $\mathbf{1 9 9 7}$ & 9.53 & 38.76 & 38.48 & 13.23 & 5.51 & 33.40 & 41.02 & 20.06 \\
\hline $\mathbf{1 9 9 8}$ & 8.59 & 35.79 & 40.45 & 15.17 & 4.72 & 30.95 & 42.36 & 21.97 \\
\hline $\mathbf{1 9 9 9}$ & 6.30 & 33.07 & 42.76 & 17.87 & 3.87 & 28.08 & 42.83 & 25.22 \\
\hline $\mathbf{2 0 0 0}$ & 5.49 & 30.09 & 43.31 & 21.10 & 4.19 & 24.74 & 42.87 & 28.20 \\
\hline $\mathbf{2 0 0 1}$ & 5.97 & 26.63 & 43.88 & 23.52 & 4.86 & 22.01 & 43.32 & 29.81 \\
\hline $\mathbf{2 0 0 2}$ & 6.16 & 23.32 & 44.12 & 26.40 & 5.14 & 18.68 & 42.78 & 33.41 \\
\hline $\mathbf{2 0 0 3}$ & 5.22 & 16.88 & 50.33 & 27.57 & 4.93 & 13.71 & 46.65 & 34.70 \\
\hline $\mathbf{2 0 0 4}$ & 4.57 & 14.05 & 49.66 & 31.72 & 4.27 & 11.08 & 45.70 & 38.94 \\
\hline $\mathbf{2 0 0 5}$ & 3.22 & 11.65 & 44.16 & 40.98 & 3.03 & 9.45 & 39.27 & 48.25 \\
\hline $\mathbf{2 0 0 6}$ & 2.62 & 9.82 & 45.53 & 42.04 & 2.74 & 8.71 & 39.43 & 49.13 \\
\hline
\end{tabular}


Savannah River Site 2006

Absence Data

Appendix C. Total Number of Workers Who Reported at Least One Absence by Gender, Age, and Job Category*

\begin{tabular}{|c|c|c|c|c|c|c|c|c|c|c|c|}
\hline \multirow{3}{*}{ Job Category } & \multicolumn{5}{|c|}{ Women } & \multicolumn{5}{|c|}{ Men } & \multirow[b]{3}{*}{ TOTAL } \\
\hline & \multicolumn{4}{|c|}{ Age Group } & \multirow[b]{2}{*}{ TOTAL } & \multicolumn{4}{|c|}{ Age Group } & \multirow[b]{2}{*}{ TOTAL } & \\
\hline & $16-29$ & 30 - 39 & $40-49$ & $50+$ & & $16-29$ & 30 - 39 & $40-49$ & $50+$ & & \\
\hline Professional & 6 & 18 & 123 & 134 & 281 & 12 & 28 & 165 & 272 & 477 & 758 \\
\hline Administrative Support & 0 & 3 & 7 & 14 & 24 & 0 & 2 & 7 & 5 & 14 & 38 \\
\hline Technical Support & 3 & 28 & 92 & 69 & 192 & 4 & 29 & 143 & 188 & 364 & 556 \\
\hline Service & 0 & 0 & 7 & 10 & 17 & 0 & 7 & 88 & 98 & 193 & 210 \\
\hline Security and Fire & 0 & 0 & 0 & 1 & 1 & 0 & 1 & 5 & 14 & 20 & 21 \\
\hline Crafts & 1 & 0 & 4 & 2 & 7 & 4 & 10 & 11 & 26 & 51 & 58 \\
\hline Line Operators & 0 & 11 & 44 & 48 & 103 & 1 & 15 & 108 & 98 & 222 & 325 \\
\hline TOTAL & 10 & 60 & 277 & 278 & 625 & 21 & 92 & 527 & 701 & 1,341 & 1,966 \\
\hline
\end{tabular}

*Only those job categories and gender/age combinations with at least one absence appear in this table.

Appendix D. Total Number of Absences by Gender, Age, and Job Category*

\begin{tabular}{|c|c|c|c|c|c|c|c|c|c|c|c|}
\hline \multirow{3}{*}{ Job Category } & \multicolumn{5}{|c|}{ Women } & \multicolumn{5}{|c|}{ Men } & \multirow[b]{3}{*}{ TOTAL } \\
\hline & \multicolumn{4}{|c|}{ Age Group } & \multirow[b]{2}{*}{ TOTAL } & \multicolumn{4}{|c|}{ Age Group } & \multirow[b]{2}{*}{ TOTAL } & \\
\hline & $16-29$ & $30-39$ & $40-49$ & $50+$ & & $16-29$ & $30-39$ & $40-49$ & $50+$ & & \\
\hline Professional & 9 & 31 & 179 & 218 & 437 & 14 & 40 & 215 & 379 & 648 & 1,085 \\
\hline Administrative Support & 0 & 3 & 20 & 21 & 44 & 0 & 2 & 12 & 5 & 19 & 63 \\
\hline Technical Support & 5 & 64 & 144 & 115 & 328 & 5 & 45 & 208 & 277 & 535 & 863 \\
\hline Service & 0 & 0 & 11 & 19 & 30 & 0 & 11 & 137 & 152 & 300 & 330 \\
\hline Security and Fire & 0 & 0 & 0 & 1 & 1 & 0 & 1 & 5 & 16 & 22 & 23 \\
\hline Crafts & 1 & 0 & 5 & 2 & 8 & 6 & 11 & 11 & 36 & 64 & 72 \\
\hline Line Operators & 0 & 20 & 96 & 104 & 220 & 3 & 27 & 192 & 142 & 364 & 584 \\
\hline TOTAL & 15 & 118 & 455 & 480 & 1,068 & 28 & 137 & 780 & 1,007 & 1,952 & 3,020 \\
\hline
\end{tabular}

*Only those job categories and gender/age combinations with at least one absence appear in this table. 
Savannah River Site 2006

Absence Data

Appendix E. Distribution of the Number of Calendar Days Missed per Absence by Gender and Age*

\begin{tabular}{|c|c|c|c|c|c|c|c|c|c|c|c|}
\hline \multirow{3}{*}{$\begin{array}{c}\text { Number of } \\
\text { Calendar Days }\end{array}$} & \multicolumn{5}{|c|}{ Women } & \multicolumn{5}{|c|}{ Men } & \multirow[b]{3}{*}{ TOTAL } \\
\hline & \multicolumn{4}{|c|}{ Age Group } & \multirow[b]{2}{*}{ TOTAL } & \multicolumn{4}{|c|}{ Age Group } & \multirow[b]{2}{*}{ TOTAL } & \\
\hline & $16-29$ & $30-39$ & $40-49$ & $50+$ & & $16-29$ & $30-39$ & $40-49$ & $50+$ & & \\
\hline$<15$ & 14 & 94 & 312 & 338 & 758 & 24 & 112 & 583 & 734 & 1,453 & 2,211 \\
\hline $15-28$ & 1 & 11 & 63 & 71 & 146 & 2 & 18 & 114 & 147 & 281 & 427 \\
\hline $29-42$ & 0 & 6 & 33 & 30 & 69 & 2 & 6 & 43 & 50 & 101 & 170 \\
\hline $43-56$ & 0 & 2 & 28 & 24 & 54 & 0 & 0 & 16 & 27 & 43 & 97 \\
\hline $57-91$ & 0 & 4 & 13 & 12 & 29 & 0 & 1 & 17 & 20 & 38 & 67 \\
\hline $92-182$ & 0 & 1 & 5 & 4 & 10 & 0 & 0 & 5 & 20 & 25 & 35 \\
\hline $183+$ & 0 & 0 & 1 & 1 & 2 & 0 & 0 & 2 & 9 & 11 & 13 \\
\hline TOTAL & 15 & 118 & 455 & 480 & 1,068 & 28 & 137 & 780 & 1,007 & 1,952 & 3,020 \\
\hline
\end{tabular}

*Only those gender/age combinations with at least one absence appear in this table. 
Savannah River Site 2006

Absence Data

Appendix F. Distribution of the Number of Calendar Days Missed per Absence by Gender and Job Category*

Women

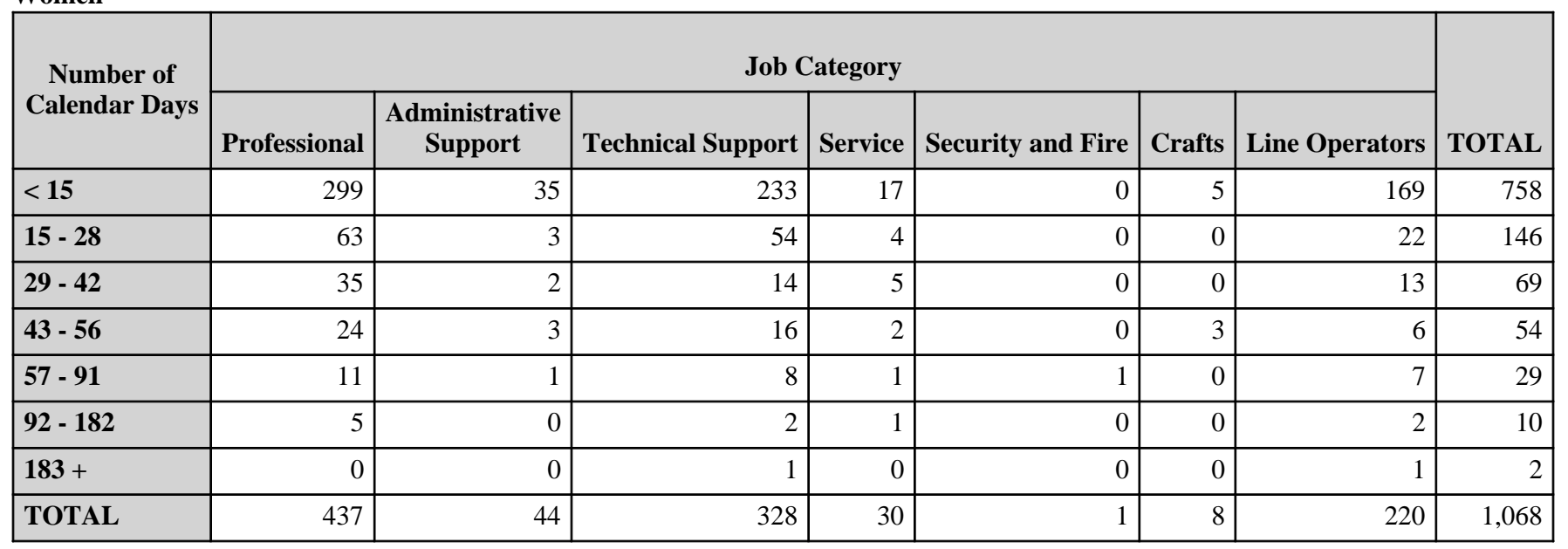

Men

\begin{tabular}{|c|c|c|c|c|c|c|c|c|}
\hline \multirow{2}{*}{$\begin{array}{c}\text { Number of } \\
\text { Calendar Days }\end{array}$} & \multicolumn{7}{|c|}{ Job Category } & \multirow[b]{2}{*}{ TOTAL } \\
\hline & Professional & $\begin{array}{l}\text { Administrative } \\
\text { Support }\end{array}$ & Technical Support & Service & Security and Fire & Crafts & Line Operators & \\
\hline$<15$ & 484 & 17 & 398 & 223 & 22 & 44 & 265 & 1,453 \\
\hline $15-28$ & 95 & 1 & 73 & 44 & 0 & 7 & 61 & 281 \\
\hline $57-91$ & 12 & 1 & 8 & 4 & 0 & 2 & 11 & 38 \\
\hline $92-182$ & 6 & 0 & 8 & 3 & 0 & 5 & 3 & 25 \\
\hline $183+$ & 3 & 0 & 5 & 2 & 0 & 0 & 1 & 11 \\
\hline TOTAL & 648 & 19 & 535 & 300 & 22 & 64 & 364 & 1,952 \\
\hline
\end{tabular}

*Only those gender/job category combinations with at least one absence appear in this table. 


\section{Savannah River Site 2006}

\section{Absence Data}

Appendix G. Number of Diagnoses in Each Diagnostic Category by Gender and Age*

\begin{tabular}{|c|c|c|c|c|c|c|}
\hline & & \multicolumn{5}{|c|}{ Women } \\
\hline & & \multicolumn{4}{|c|}{ Age Group } & \multirow[b]{2}{*}{ TOTAL } \\
\hline & & $16-29$ & $30-39$ & $40-49$ & $\mathbf{5 0}+$ & \\
\hline Diagnostic Category & ICD-9-CM Code & \multirow[b]{2}{*}{2} & \multirow[b]{2}{*}{1} & \multirow[b]{2}{*}{23} & \multirow[b]{2}{*}{34} & \multirow[b]{2}{*}{60} \\
\hline INFECTIOUS \& PARASITIC DISEASES (DIS) & 001-139 & & & & & \\
\hline -Intestinal Infectious Dis & 001-009 & 0 & 0 & 12 & 13 & 25 \\
\hline -Other Bacterial Dis & $030-041$ & 1 & 1 & 6 & 12 & 20 \\
\hline -Viral Dis with Exanthem & $050-057$ & 1 & 0 & 0 & 1 & 2 \\
\hline -Other Viral Dis \& Chlamydiae & 070-079 & 0 & 0 & 5 & 2 & 7 \\
\hline -Syphilis \& Other Venereal Dis & 090-099 & 0 & 0 & 0 & 0 & 0 \\
\hline -Mycoses & $110-118$ & 0 & 0 & 0 & 1 & 1 \\
\hline -Other Infections \& Parasitic Dis & 130-136 & 0 & 0 & 0 & 5 & 5 \\
\hline MALIGNANT NEOPLASMS & $140-208,230-234$ & 0 & 0 & 5 & 12 & 17 \\
\hline -Lip, Oral Cavity, Pharynx & $140-149$ & 0 & 0 & 0 & 2 & 2 \\
\hline -Digestive \& Peritoneal & $150-159$ & 0 & 0 & 0 & 2 & 2 \\
\hline -Respiratory \& Intrathoracic & $160-165$ & 0 & 0 & 0 & 0 & 0 \\
\hline -Bone, Connective Tissue, Skin & 170-173, 176 & 0 & 0 & 1 & 1 & 2 \\
\hline -Breast & 174-175 & 0 & 0 & 0 & 4 & 4 \\
\hline -Genitourinary & 179-189 & 0 & 0 & 0 & 0 & 0 \\
\hline -Other \& Unspecified Sites & 190, 193-199 & 0 & 0 & 4 & 3 & 7 \\
\hline -Lymphatic \& Hematopoietic & $200-208$ & 0 & 0 & 0 & 0 & 0 \\
\hline BENIGN \& UNCERTAIN NEOPLASMS & 210-229, 235-239 & 0 & 4 & 26 & 15 & 45 \\
\hline ENDOCRINE/METABOLIC/IMMUNITY & $240-279$ & 0 & 1 & 20 & 21 & 42 \\
\hline -Thyroid Gland Disorders & $240-246$ & 0 & 0 & 8 & 5 & 13 \\
\hline -Other Endocrine Gland Dis & 250-259 & 0 & 1 & 1 & 8 & 10 \\
\hline -Other Metabolic \& Immunity Disorders & $270-279$ & 0 & 0 & 11 & 8 & 19 \\
\hline BLOOD \& BLOOD-FORMING ORGANS & $280-289$ & 0 & 2 & 9 & 1 & 12 \\
\hline MENTAL DISORDERS & $290-319$ & 0 & 5 & 12 & 9 & 26 \\
\hline -Psychoses & 290-299 & 0 & 1 & 2 & 2 & 5 \\
\hline -Non-Psychotic Disorders & $300-302,306-316$ & 0 & 4 & 10 & 7 & 21 \\
\hline -Alcohol Dependence & 303 & 0 & 0 & 0 & 0 & 0 \\
\hline -Drug Dependence & 304-305 & 0 & 0 & 0 & 0 & 0 \\
\hline NERVOUS SYSTEM (NS) \& SENSE ORGANS & $320-389$ & 1 & 11 & 45 & 42 & 99 \\
\hline -Inflammatory Dis of Central NS & $320-326$ & 0 & 0 & 0 & 0 & 0 \\
\hline -Hereditary/Degenerative Central NS Dis & 330-337 & 0 & 0 & 0 & 0 & 0 \\
\hline -Other Disorders of Central NS & $340-349$ & 0 & 6 & 12 & 6 & 24 \\
\hline -Disorders of Peripheral NS & $350-359$ & 0 & 5 & 11 & 12 & 28 \\
\hline -Disorders of Eye & $360-379$ & 1 & 0 & 9 & 17 & 27 \\
\hline -Dis of Ear \& Mastoid & $380-389$ & 0 & 0 & 13 & 6 & 19 \\
\hline CIRCULATORY SYSTEM & $390-459$ & 0 & 9 & 26 & 24 & 59 \\
\hline -Hypertensive Dis & $401-405$ & 0 & 1 & 19 & 6 & 26 \\
\hline
\end{tabular}

*Only those diagnostic categories and gender/age combinations with at least one occurrence appear in this table. 


\section{Savannah River Site 2006}

\section{Absence Data}

Appendix G. Number of Diagnoses in Each Diagnostic Category by Gender and Age*

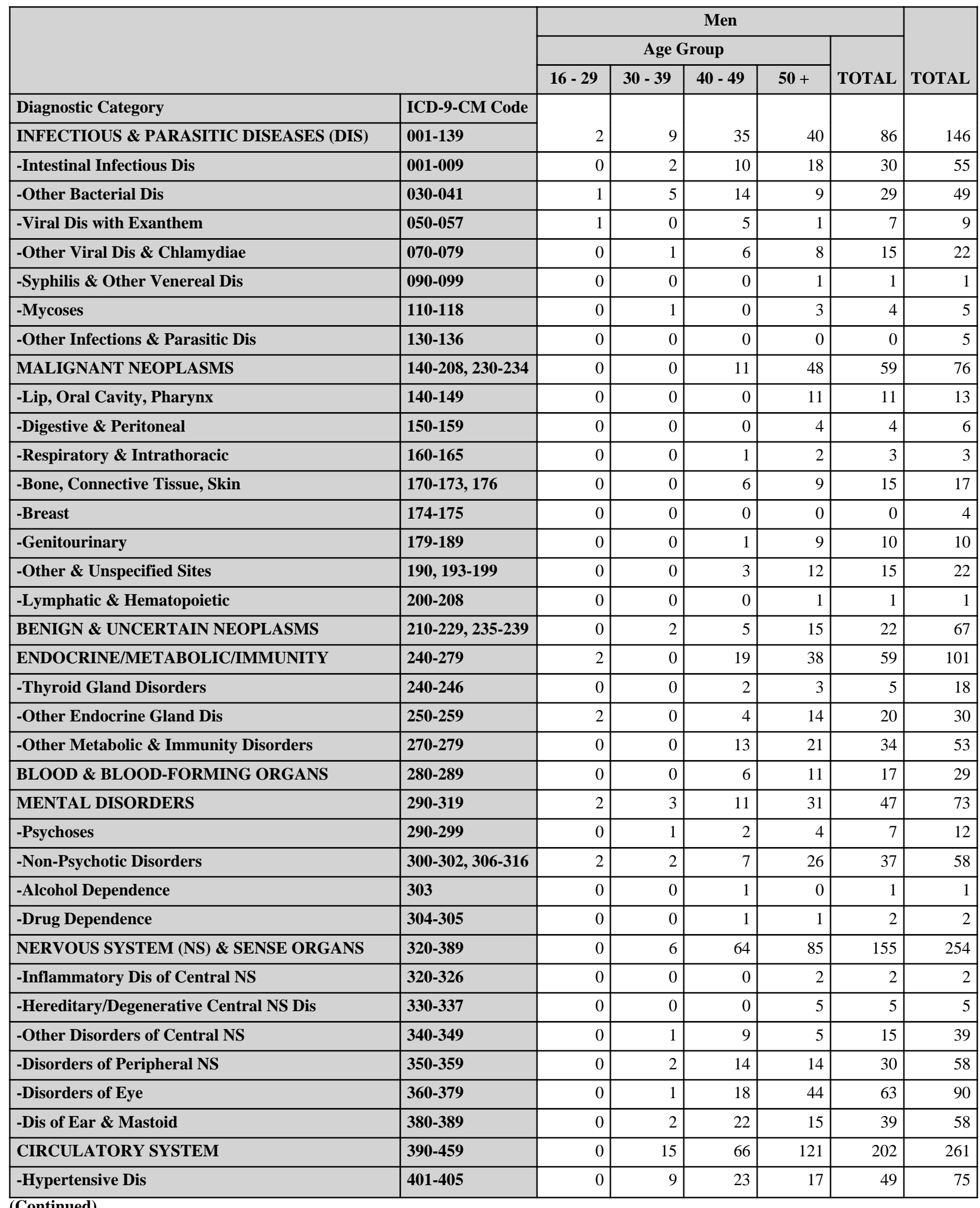

*Only those diagnostic categories and gender/age combinations with at least one occurrence appear in this table. 


\section{Savannah River Site 2006}

\section{Absence Data}

Appendix G. Number of Diagnoses in Each Diagnostic Category by Gender and Age*

\begin{tabular}{|c|c|c|c|c|c|c|}
\hline & & \multicolumn{5}{|c|}{ Women } \\
\hline & & \multicolumn{4}{|c|}{ Age Group } & \multirow[b]{2}{*}{ TOTAL } \\
\hline & & $16-29$ & $30-39$ & $40-49$ & $50+$ & \\
\hline Diagnostic Category & ICD-9-CM Code & \multirow[b]{2}{*}{0} & \multirow[b]{2}{*}{0} & \multirow[b]{2}{*}{2} & \multirow[b]{2}{*}{3} & \multirow[b]{2}{*}{5} \\
\hline -Ischemic Heart Dis & $410-414$ & & & & & \\
\hline -Dis of Pulmonary Circulation & $415-417$ & 0 & 0 & 0 & 1 & 1 \\
\hline -Other Heart Dis & $420-429$ & 0 & 0 & 2 & 7 & 9 \\
\hline -Cerebrovascular Dis & $430-438$ & 0 & 0 & 0 & 1 & 1 \\
\hline -Dis of Arteries \& Capillaries & $440-448$ & 0 & 0 & 2 & 0 & 2 \\
\hline -Dis of Veins, Lymphatics, Other & 451-459 & 0 & 8 & 1 & 6 & 15 \\
\hline RESPIRATORY SYSTEM & $460-519$ & 6 & 25 & 168 & 189 & 388 \\
\hline -Acute Respiratory Infections & $460-466$ & 3 & 14 & 108 & 133 & 258 \\
\hline -Other Dis Upper Respiratory Tract & \begin{tabular}{|l|}
$470-478$ \\
\end{tabular} & 2 & 4 & 33 & 30 & 69 \\
\hline -Pneumonia \& Influenza & $480-487$ & 1 & 6 & 20 & 15 & 42 \\
\hline -Chronic Obstructive Dis & $490-496$ & 0 & 1 & 7 & 9 & 17 \\
\hline -Lung Dis from External Agents & \begin{tabular}{|l|}
$500-508$ \\
\end{tabular} & 0 & 0 & 0 & 0 & 0 \\
\hline -Other Respiratory Dis & $510-519$ & 0 & 0 & 0 & 2 & 2 \\
\hline DIGESTIVE SYSTEM & \begin{tabular}{|l|}
$520-579$ \\
\end{tabular} & 0 & 17 & 35 & 54 & 106 \\
\hline -Oral Cavity, Saliva Glands, Jaw & $520-529$ & 0 & 4 & 16 & 4 & 24 \\
\hline -Esophagus, Stomach, Duodenum & $530-537$ & 0 & 2 & 4 & 19 & 25 \\
\hline -Appendicitis & \begin{tabular}{|l|}
$540-543$ \\
\end{tabular} & 0 & 0 & 2 & 3 & 5 \\
\hline -Hernia & $550-553$ & 0 & 0 & 0 & 4 & 4 \\
\hline -Enteritis, Colitis & $555-558$ & 0 & 4 & 4 & 6 & 14 \\
\hline -Other Intestinal Dis & $560-569$ & 0 & 7 & 5 & 13 & 25 \\
\hline -Other Digestive Dis & $570-579$ & 0 & 0 & 4 & 5 & 9 \\
\hline GENITOURINARY SYSTEM & $580-629$ & 1 & 23 & 79 & 34 & 137 \\
\hline -Other Urinary Dis & $590-599$ & 0 & 13 & 18 & 10 & 41 \\
\hline -Male Genital Organ Dis & $600-608$ & 0 & 0 & 0 & 0 & 0 \\
\hline -Breast Disorders & $610-611$ & 1 & 0 & 5 & 3 & 9 \\
\hline -Pelvic Inflammatory Dis & 614-616 & 0 & 1 & 3 & 0 & 4 \\
\hline -Other Female Disorders & $617-629$ & 0 & 9 & 53 & 21 & 83 \\
\hline PREGNANCY \& CHILDBIRTH & $630-677$ & 0 & 8 & 0 & 0 & 8 \\
\hline -Pregnancy with Abortive Outcome & $634-639$ & 0 & 8 & 0 & 0 & 8 \\
\hline SKIN \& SUBCUTANEOUS TISSUE & $680-709$ & 0 & 3 & 6 & 8 & 17 \\
\hline -Infections & $680-686$ & 0 & 0 & 1 & 2 & 3 \\
\hline -Other Inflammatory Conditions & $690-698$ & 0 & 0 & 0 & 0 & 0 \\
\hline -Other & 700-709 & 0 & 3 & 5 & 6 & 14 \\
\hline $\begin{array}{l}\text { MUSCULOSKELETAL \& CONNECTIVE } \\
\text { TISSUE }\end{array}$ & $710-739$ & 4 & 21 & 88 & 176 & 289 \\
\hline -Arthropathies & $710-719$ & 1 & 4 & 13 & 39 & 57 \\
\hline -Dorsopathies & $720-724$ & 3 & 16 & 55 & 82 & 156 \\
\hline
\end{tabular}

(Continued)

*Only those diagnostic categories and gender/age combinations with at least one occurrence appear in this table. 


\section{Savannah River Site 2006}

\section{Absence Data}

Appendix G. Number of Diagnoses in Each Diagnostic Category by Gender and Age*

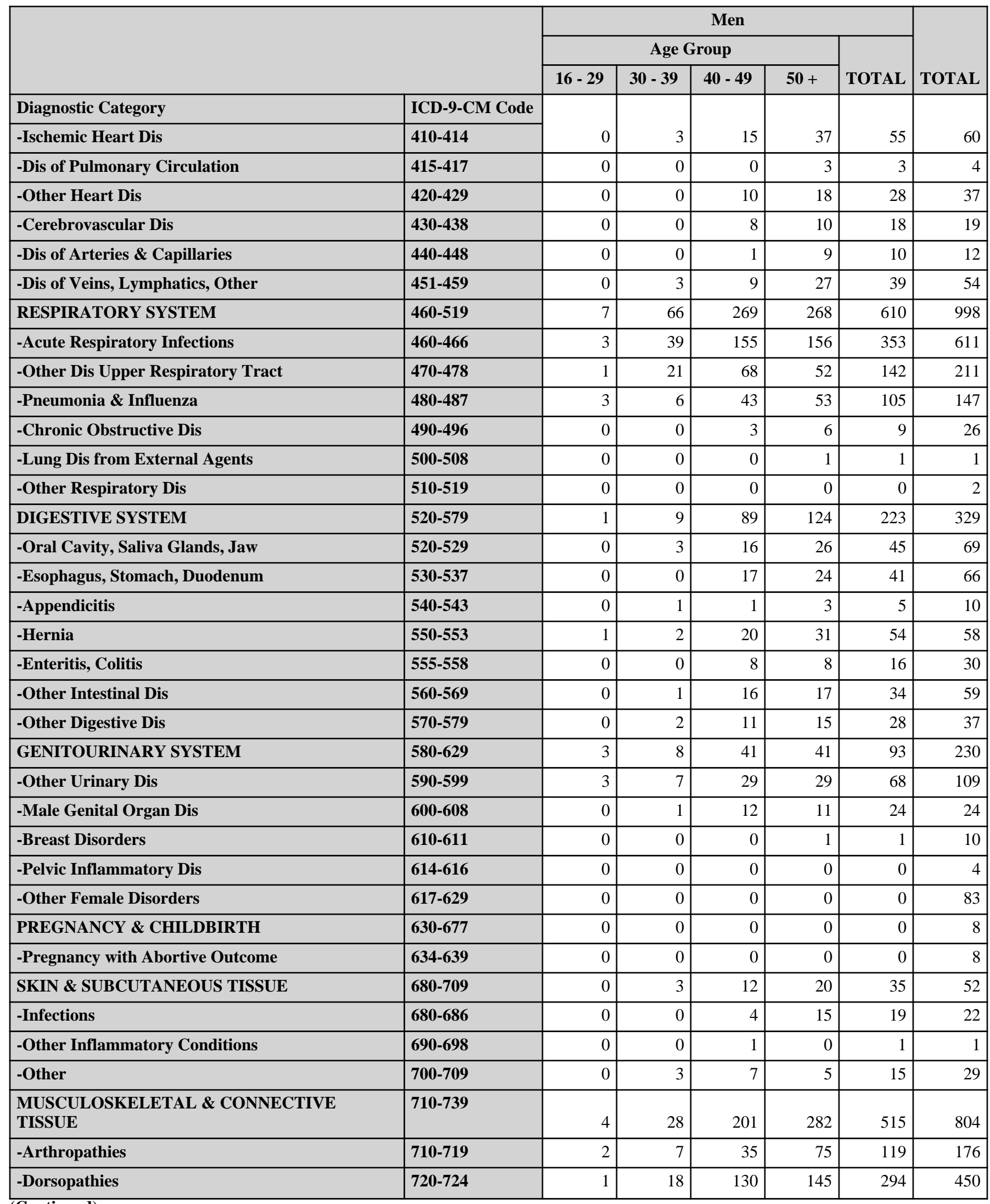

(Continued)

*Only those diagnostic categories and gender/age combinations with at least one occurrence appear in this table. 


\section{Savannah River Site 2006}

\section{Absence Data}

Appendix G. Number of Diagnoses in Each Diagnostic Category by Gender and Age*

\begin{tabular}{|c|c|c|c|c|c|c|}
\hline & & \multicolumn{5}{|c|}{ Women } \\
\hline & & \multicolumn{4}{|c|}{ Age Group } & \multirow[b]{2}{*}{ TOTAL } \\
\hline & & $16-29$ & $30-39$ & $40-49$ & $50+$ & \\
\hline Diagnostic Category & ICD-9-CM Code & \multirow[b]{2}{*}{0} & \multirow[b]{2}{*}{1} & \multirow[b]{2}{*}{15} & \multirow[b]{2}{*}{47} & \multirow[b]{2}{*}{63} \\
\hline -Rheumatism, Excluding Back & 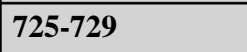 & & & & & \\
\hline -Other Dis \& Acquired Deformities & $730-739$ & 0 & 0 & 5 & 8 & 13 \\
\hline CONGENITAL ANOMALIES & 740-759 & 0 & 0 & 0 & 1 & 1 \\
\hline $\begin{array}{l}\text { SYMPTOMS, SIGNS, \& ILL-DEFINED } \\
\text { CONDITIONS }\end{array}$ & \begin{tabular}{|l|}
$780-799$ \\
\end{tabular} & 3 & 35 & 144 & 121 & 303 \\
\hline -Symptoms & $780-789$ & 3 & 34 & 141 & 117 & 295 \\
\hline -Non-Specific Abnormal Findings & $790-796$ & 0 & 1 & 3 & 4 & 8 \\
\hline -Ill-Defined \& Unknown Causes & 797-799 & 0 & 0 & 0 & 0 & 0 \\
\hline INJURY \& POISONING & $800-999$ & 3 & 12 & 52 & 33 & 100 \\
\hline -Fracture - Skull & $800-804$ & 0 & 0 & 3 & 0 & 3 \\
\hline -Fracture - Neck, Trunk & \begin{tabular}{|l|}
$805-809$ \\
\end{tabular} & 0 & 0 & 0 & 0 & 0 \\
\hline -Fracture - Upper Limb & \begin{tabular}{|l|}
$810-819$ \\
\end{tabular} & 0 & 1 & 1 & 0 & 2 \\
\hline -Fracture - Lower Limb & 820-829 & 0 & 0 & 2 & 3 & 5 \\
\hline -Dislocation & \begin{tabular}{|l|}
$830-839$ \\
\end{tabular} & 0 & 1 & 5 & 5 & 11 \\
\hline -Sprains \& Strains - Back & \begin{tabular}{|l|}
$846-847$ \\
\end{tabular} & 2 & 2 & 5 & 3 & 12 \\
\hline -Sprains \& Strains - Other & $840-845,848$ & 0 & 1 & 9 & 5 & 15 \\
\hline -Intracranial Injury & $850-854$ & 0 & 0 & 1 & 0 & 1 \\
\hline -Internal Injury - Thorax, Abdomen, Pelvis & $860-869$ & 0 & 0 & 0 & 0 & 0 \\
\hline -Open Wound - Head, Neck, Trunk & \begin{tabular}{|l|}
$870-879$ \\
\end{tabular} & 0 & 0 & 1 & 1 & 2 \\
\hline -Open Wound - Upper Limb & $880-887$ & 0 & 0 & 1 & 0 & 1 \\
\hline -Open Wound - Lower Limb & $890-897$ & 0 & 0 & 1 & 0 & 1 \\
\hline -Late Effects of Accident & \begin{tabular}{|c|}
$905-909$ \\
\end{tabular} & 0 & 0 & 1 & 0 & 1 \\
\hline -Superficial Injury & $910-919$ & 0 & 0 & 1 & 0 & 1 \\
\hline -Contusion & $920-924$ & 1 & 0 & 0 & 0 & 1 \\
\hline -Crushing Injury & $925-929$ & 0 & 0 & 1 & 0 & 1 \\
\hline -Foreign Body Entering Orifice & 930-939 & 0 & 0 & 0 & 0 & 0 \\
\hline -Burns & $940-949$ & 0 & 0 & 6 & 0 & 6 \\
\hline -Complications \& Unspecified Injuries & 958-959 & 0 & 0 & 0 & 0 & 0 \\
\hline -Toxic Effects - Non-medicinal & $980-989$ & 0 & 0 & 0 & 0 & 0 \\
\hline -Unspecified Effects - External Causes & $990-995$ & 0 & 0 & 6 & 2 & 8 \\
\hline -Complications of Surgical/Medical Care & 996-999 & 0 & 7 & 8 & 14 & 29 \\
\hline $\begin{array}{l}\text { HEALTH STATUS/HEALTH SERVICE } \\
\text { CONTACT }\end{array}$ & V01-V82 & 5 & 14 & 8 & 14 & 41 \\
\hline -Personal \& Family History & V10-V19 & 0 & 0 & 2 & 6 & 8 \\
\hline -Health Services Reproduction/Development & V20-V29 & 5 & 13 & 5 & 0 & 23 \\
\hline -Specific Procedures/Aftercare & V50-V59 & 0 & 0 & 1 & 8 & 9 \\
\hline
\end{tabular}

(Continued)

*Only those diagnostic categories and gender/age combinations with at least one occurrence appear in this table. 


\section{Savannah River Site 2006}

\section{Absence Data}

Appendix G. Number of Diagnoses in Each Diagnostic Category by Gender and Age*

\begin{tabular}{|c|c|c|c|c|c|c|c|}
\hline & & \multicolumn{5}{|c|}{ Men } & \multirow[b]{3}{*}{ TOTAL } \\
\hline & & \multicolumn{4}{|c|}{ Age Group } & \multirow[b]{2}{*}{ TOTAL } & \\
\hline & & $16-29$ & 30 - 39 & $40-49$ & $50+$ & & \\
\hline -Rheumatism, Excluding Back & 725-729 & 1 & 2 & 30 & 57 & 90 & 153 \\
\hline -Other Dis \& Acquired Deformities & 730-739 & 0 & 1 & 6 & 5 & 12 & 25 \\
\hline $\begin{array}{l}\text { SYMPTOMS, SIGNS, \& ILL-DEFINED } \\
\text { CONDITIONS }\end{array}$ & 780-799 & 5 & 39 & 190 & 230 & 464 & 767 \\
\hline -Symptoms & 780-789 & 5 & 39 & 184 & 217 & 445 & 740 \\
\hline -Non-Specific Abnormal Findings & $790-796$ & 0 & 0 & 6 & 11 & 17 & 25 \\
\hline -Fracture - Neck, Trunk & 805-809 & 0 & 0 & 2 & 6 & 8 & 8 \\
\hline -Fracture - Upper Limb & 810-819 & 1 & 0 & 5 & 3 & 9 & 11 \\
\hline -Fracture - Lower Limb & 820-829 & 0 & 0 & 3 & 8 & 11 & 16 \\
\hline -Dislocation & 830-839 & 3 & 2 & 25 & 21 & 51 & 62 \\
\hline -Sprains \& Strains - Back & 846-847 & 0 & 6 & 7 & 10 & 23 & 35 \\
\hline -Sprains \& Strains - Other & $840-845,848$ & 0 & 2 & 21 & 28 & 51 & 66 \\
\hline -Intracranial Injury & $850-854$ & 0 & 0 & 1 & 1 & 2 & 3 \\
\hline -Internal Injury - Thorax, Abdomen, Pelvis & $860-869$ & 0 & 0 & 2 & 0 & 2 & 2 \\
\hline -Contusion & $920-924$ & 0 & 3 & 3 & 10 & 16 & 17 \\
\hline -Crushing Injury & 925-929 & 0 & 0 & 0 & 0 & 0 & 1 \\
\hline -Foreign Body Entering Orifice & 930-939 & 0 & 0 & 0 & 2 & 2 & 2 \\
\hline -Burns & 940-949 & 1 & 0 & 3 & 1 & 5 & 11 \\
\hline -Complications \& Unspecified Injuries & 958-959 & 0 & 0 & 1 & 0 & 1 & 1 \\
\hline -Toxic Effects - Non-medicinal & 980-989 & 1 & 2 & 1 & 0 & 4 & 4 \\
\hline -Unspecified Effects - External Causes & $990-995$ & 1 & 1 & 11 & 3 & 16 & 24 \\
\hline -Complications of Surgical/Medical Care & 996-999 & 0 & 0 & 15 & 19 & 34 & 63 \\
\hline $\begin{array}{l}\text { HEALTH STATUS/HEALTH SERVICE } \\
\text { CONTACT }\end{array}$ & V01-V82 & 1 & 1 & 17 & 23 & 42 & 83 \\
\hline -Personal \& Family History & V10-V19 & 0 & 0 & 2 & 2 & 4 & 12 \\
\hline -Health Services Reproduction/Development & V20-V29 & 1 & 1 & 0 & 0 & 2 & 25 \\
\hline -Specific Procedures/Aftercare & V50-V59 & 0 & 0 & 6 & 15 & 21 & 30 \\
\hline
\end{tabular}

(Continued)

*Only those diagnostic categories and gender/age combinations with at least one occurrence appear in this table. 
Savannah River Site 2006

Absence Data

Appendix G. Number of Diagnoses in Each Diagnostic Category by Gender and Age*

\begin{tabular}{|c|c|c|c|c|c|c|}
\hline & & \multicolumn{5}{|c|}{ Women } \\
\hline & & \multicolumn{4}{|c|}{ Age Group } & \multirow[b]{2}{*}{ TOTAL } \\
\hline & & $16-29$ & $30-39$ & $40-49$ & $50+$ & \\
\hline Diagnostic Category & ICD-9-CM Code & \multirow[b]{2}{*}{0} & \multirow[b]{2}{*}{0} & \multirow[b]{2}{*}{0} & \multirow[b]{2}{*}{0} & \multirow[b]{2}{*}{0} \\
\hline -Other Circumstances & V60-V69 & & & & & \\
\hline -Examination \& Investigation & V70-V82 & 0 & 1 & 0 & 0 & 1 \\
\hline
\end{tabular}

*Only those diagnostic categories and gender/age combinations with at least one occurrence appear in this table. 
Savannah River Site 2006

Absence Data

Appendix G. Number of Diagnoses in Each Diagnostic Category by Gender and Age*

\begin{tabular}{|c|c|c|c|c|c|c|c|}
\hline & & \multicolumn{5}{|c|}{ Men } & \multirow[b]{3}{*}{ TOTAL } \\
\hline & & \multicolumn{4}{|c|}{ Age Group } & \multirow[b]{2}{*}{ TOTAL } & \\
\hline & & $16-29$ & $30-39$ & $40-49$ & $50+$ & & \\
\hline -Other Circumstances & V60-V69 & 0 & 0 & 3 & 2 & 5 & 5 \\
\hline -Examination \& Investigation & V70-V82 & 0 & 0 & 6 & 4 & 10 & 11 \\
\hline
\end{tabular}

\begin{tabular}{|c|c|c|c|c|c|c|c|c|c|c|c|}
\hline & \multicolumn{5}{|c|}{ Women } & \multicolumn{5}{|c|}{ Men } & \multirow[b]{3}{*}{ TOTAL } \\
\hline & \multicolumn{4}{|c|}{ Age Group } & \multirow[b]{2}{*}{ TOTAL } & \multicolumn{4}{|c|}{ Age Group } & \multirow[b]{2}{*}{ TOTAL } & \\
\hline & $16-29$ & $30-39$ & $40-49$ & $50+$ & & $16-29$ & $30-39$ & $40-49$ & $50+$ & & \\
\hline Diagnostic Category & \multirow[b]{2}{*}{25} & \multirow[b]{2}{*}{191} & \multirow[b]{2}{*}{746} & \multirow[b]{2}{*}{788} & \multirow[b]{2}{*}{1,750} & \multirow[b]{2}{*}{35} & \multirow[b]{2}{*}{206} & \multirow[b]{2}{*}{1,155} & \multirow[b]{2}{*}{1,512} & \multirow[b]{2}{*}{2,908} & \multirow[b]{2}{*}{4,658} \\
\hline TOTAL & & & & & & & & & & & \\
\hline
\end{tabular}

*Only those diagnostic categories and gender/age combinations with at least one occurrence appear in this table. 
Savannah River Site 2006

Absence Data

Appendix H. Total Number of Calendar Days Absent in Each Diagnostic Category by Gender and Age*

\begin{tabular}{|c|c|c|c|c|c|c|}
\hline & & \multicolumn{5}{|c|}{ Women } \\
\hline & & \multicolumn{4}{|c|}{ Age Group } & \multirow[b]{2}{*}{ TOTAL } \\
\hline & & $16-29$ & $30-39$ & $40-49$ & $50+$ & \\
\hline Diagnostic Category & ICD-9-CM Code & \multirow[b]{2}{*}{17} & \multirow[b]{2}{*}{10} & \multirow[b]{2}{*}{233} & \multirow[b]{2}{*}{263} & \multirow[b]{2}{*}{523} \\
\hline INFECTIOUS \& PARASITIC DISEASES (DIS) & 001-139 & & & & & \\
\hline MALIGNANT NEOPLASMS & $140-208,230-234$ & 0 & 0 & 52 & 184 & 236 \\
\hline BENIGN \& UNCERTAIN NEOPLASMS & $210-229,235-239$ & 0 & 170 & 927 & 484 & 1,581 \\
\hline ENDOCRINE/METABOLIC/IMMUNITY & $240-279$ & 0 & 3 & 317 & 319 & 639 \\
\hline BLOOD \& BLOOD-FORMING ORGANS & $280-289$ & 0 & 16 & 140 & 42 & 198 \\
\hline MENTAL DISORDERS & 290-319 & 0 & 217 & 378 & 436 & 1,031 \\
\hline NERVOUS SYSTEM (NS) \& SENSE ORGANS & $320-389$ & 5 & 157 & 845 & 631 & 1,638 \\
\hline CIRCULATORY SYSTEM & $390-459$ & 0 & 69 & 395 & 672 & 1,136 \\
\hline RESPIRATORY SYSTEM & $460-519$ & 32 & 201 & 1,799 & 1,786 & 3,818 \\
\hline DIGESTIVE SYSTEM & $520-579$ & 0 & 242 & 450 & 944 & 1,636 \\
\hline GENITOURINARY SYSTEM & $580-629$ & 7 & 231 & 1,854 & 588 & 2,680 \\
\hline PREGNANCY \& CHILDBIRTH & $630-677$ & 0 & 171 & 0 & 0 & 171 \\
\hline SKIN \& SUBCUTANEOUS TISSUE & $680-709$ & 0 & 39 & 104 & 106 & 249 \\
\hline $\begin{array}{l}\text { MUSCULOSKELETAL \& CONNECTIVE } \\
\text { TISSUE }\end{array}$ & 710-739 & 25 & 259 & 1,717 & 2,399 & 4,400 \\
\hline CONGENITAL ANOMALIES & 740-759 & 0 & 0 & 0 & 10 & 10 \\
\hline $\begin{array}{l}\text { SYMPTOMS, SIGNS, \& ILL-DEFINED } \\
\text { CONDITIONS }\end{array}$ & \begin{tabular}{|c|}
$780-799$ \\
\end{tabular} & 12 & 286 & 880 & 958 & 2,136 \\
\hline INJURY \& POISONING & $800-999$ & 18 & 115 & 899 & 815 & 1,847 \\
\hline
\end{tabular}

\footnotetext{
*Absences with >1 ICD-9-CM code in the same diagnostic category were counted only once. Only those diagnostic categories and gender/age combinations with at least one occurrence appear in this table.
} 
Savannah River Site 2006

Absence Data

Appendix H. Total Number of Calendar Days Absent in Each Diagnostic Category by Gender and Age*

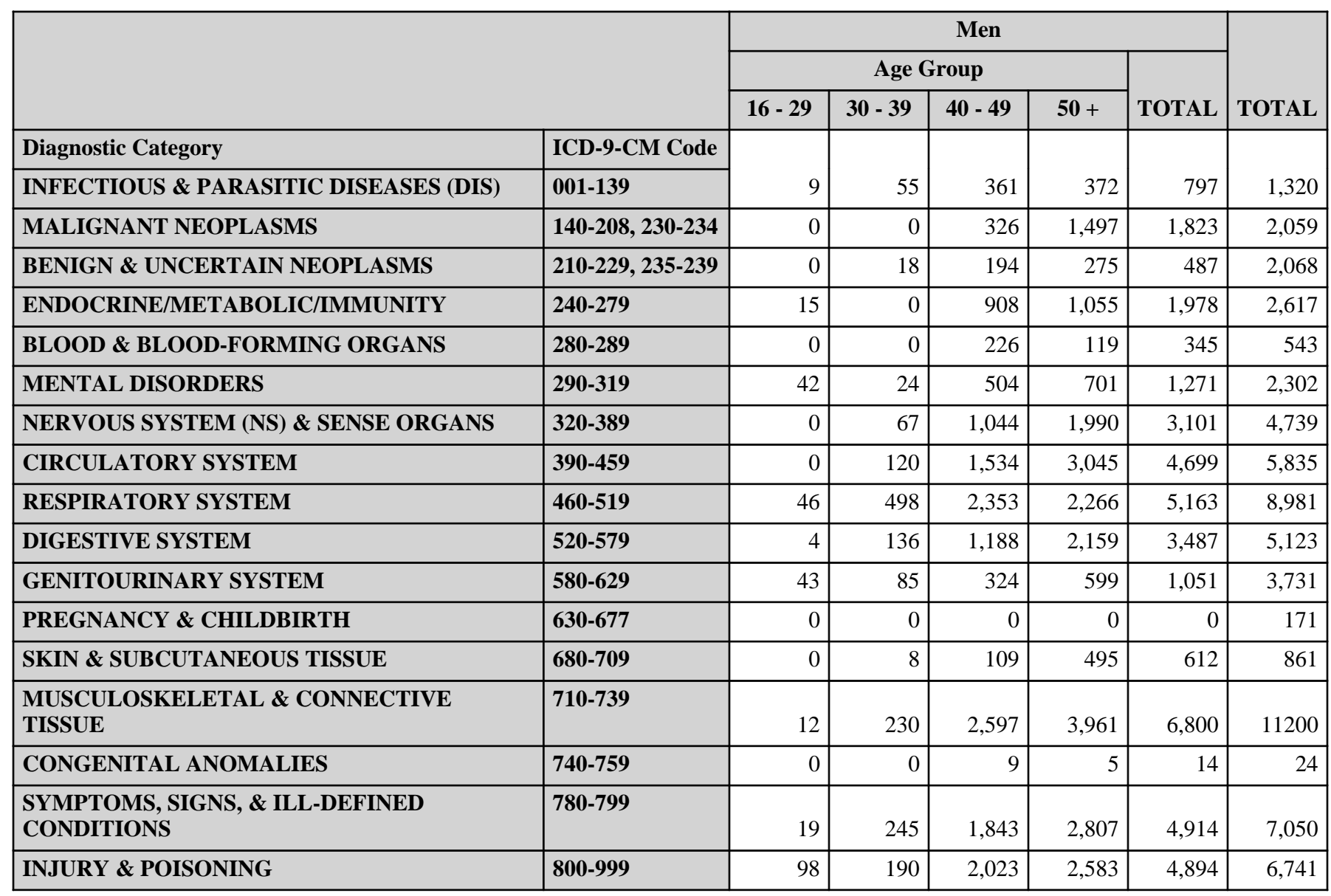

\footnotetext{
*Absences with >1 ICD-9-CM code in the same diagnostic category were counted only once. Only those diagnostic categories and gender/age combinations with at least one occurrence appear in this table.
} 


\section{Savannah River Site 2006}

\section{Absence Data}

Appendix I. Number of Diagnoses in Each Diagnostic Category by Gender and Job Category*

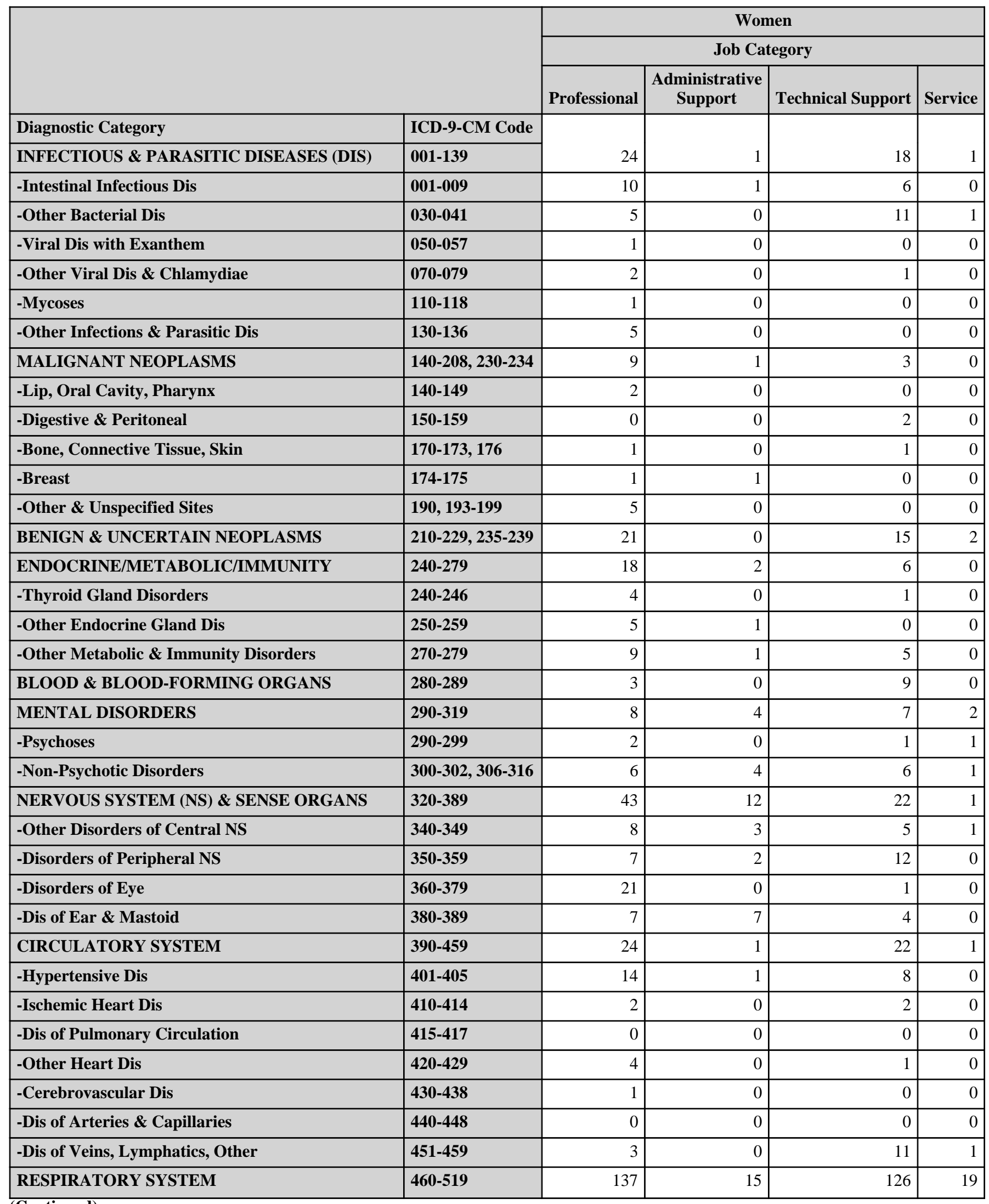

(Continued)

*Only those diagnostic categories and gender/job category combinations with at least one occurrence appear in this table. 


\section{Savannah River Site 2006}

\section{Absence Data}

Appendix I. Number of Diagnoses in Each Diagnostic Category by Gender and Job Category*

\begin{tabular}{|c|c|c|c|c|c|}
\hline & & \multicolumn{4}{|c|}{ Women } \\
\hline & & \multicolumn{3}{|c|}{ Job Category } & \multirow[b]{2}{*}{ TOTAL } \\
\hline & & Security and Fire & Crafts & Line Operators & \\
\hline Diagnostic Category & ICD-9-CM Code & \multirow[b]{2}{*}{0} & \multirow[b]{2}{*}{0} & \multirow[b]{2}{*}{16} & \multirow[b]{2}{*}{60} \\
\hline INFECTIOUS \& PARASITIC DISEASES (DIS) & 001-139 & & & & \\
\hline -Intestinal Infectious Dis & 001-009 & 0 & 0 & 8 & 25 \\
\hline -Other Bacterial Dis & 030-041 & 0 & 0 & 3 & 20 \\
\hline -Viral Dis with Exanthem & 050-057 & 0 & 0 & 1 & 2 \\
\hline -Other Viral Dis \& Chlamydiae & 070-079 & 0 & 0 & 4 & 7 \\
\hline -Mycoses & $110-118$ & 0 & 0 & 0 & 1 \\
\hline -Other Infections \& Parasitic Dis & $130-136$ & 0 & 0 & 0 & 5 \\
\hline MALIGNANT NEOPLASMS & $140-208,230-234$ & 0 & 1 & 3 & 17 \\
\hline -Lip, Oral Cavity, Pharynx & 140-149 & 0 & 0 & 0 & 2 \\
\hline -Digestive \& Peritoneal & $150-159$ & 0 & 0 & 0 & 2 \\
\hline -Bone, Connective Tissue, Skin & $170-173,176$ & 0 & 0 & 0 & 2 \\
\hline -Breast & 174-175 & 0 & 0 & 2 & 4 \\
\hline -Other \& Unspecified Sites & 190, 193-199 & 0 & 1 & 1 & 7 \\
\hline BENIGN \& UNCERTAIN NEOPLASMS & 210-229, 235-239 & 1 & 1 & 5 & 45 \\
\hline ENDOCRINE/METABOLIC/IMMUNITY & $240-279$ & 0 & 0 & 16 & 42 \\
\hline -Thyroid Gland Disorders & $240-246$ & 0 & 0 & 8 & 13 \\
\hline -Other Endocrine Gland Dis & $250-259$ & 0 & 0 & 4 & 10 \\
\hline -Other Metabolic \& Immunity Disorders & $270-279$ & 0 & 0 & 4 & 19 \\
\hline BLOOD \& BLOOD-FORMING ORGANS & $280-289$ & 0 & 0 & 0 & 12 \\
\hline MENTAL DISORDERS & $290-319$ & 0 & 0 & 5 & 26 \\
\hline -Psychoses & $290-299$ & 0 & 0 & 1 & 5 \\
\hline -Non-Psychotic Disorders & $300-302,306-316$ & 0 & 0 & 4 & 21 \\
\hline NERVOUS SYSTEM (NS) \& SENSE ORGANS & $320-389$ & 0 & 0 & 21 & 99 \\
\hline -Other Disorders of Central NS & 340-349 & 0 & 0 & 7 & 24 \\
\hline -Disorders of Peripheral NS & $350-359$ & 0 & 0 & 7 & 28 \\
\hline -Disorders of Eye & $360-379$ & 0 & 0 & 5 & 27 \\
\hline -Dis of Ear \& Mastoid & $380-389$ & 0 & 0 & 1 & 19 \\
\hline CIRCULATORY SYSTEM & $390-459$ & 0 & 0 & 11 & 59 \\
\hline -Hypertensive Dis & $401-405$ & 0 & 0 & 3 & 26 \\
\hline -Ischemic Heart Dis & $410-414$ & 0 & 0 & 1 & 5 \\
\hline -Dis of Pulmonary Circulation & $415-417$ & 0 & 0 & 1 & 1 \\
\hline -Other Heart Dis & $420-429$ & 0 & 0 & 4 & 9 \\
\hline -Cerebrovascular Dis & $430-438$ & 0 & 0 & 0 & 1 \\
\hline -Dis of Arteries \& Capillaries & $440-448$ & 0 & 0 & 2 & 2 \\
\hline -Dis of Veins, Lymphatics, Other & $451-459$ & 0 & 0 & 0 & 15 \\
\hline RESPIRATORY SYSTEM & $460-519$ & 0 & 2 & 89 & 388 \\
\hline
\end{tabular}

(Continued)

*Only those diagnostic categories and gender/job category combinations with at least one occurrence appear in this table. 


\section{Savannah River Site 2006}

\section{Absence Data}

Appendix I. Number of Diagnoses in Each Diagnostic Category by Gender and Job Category*

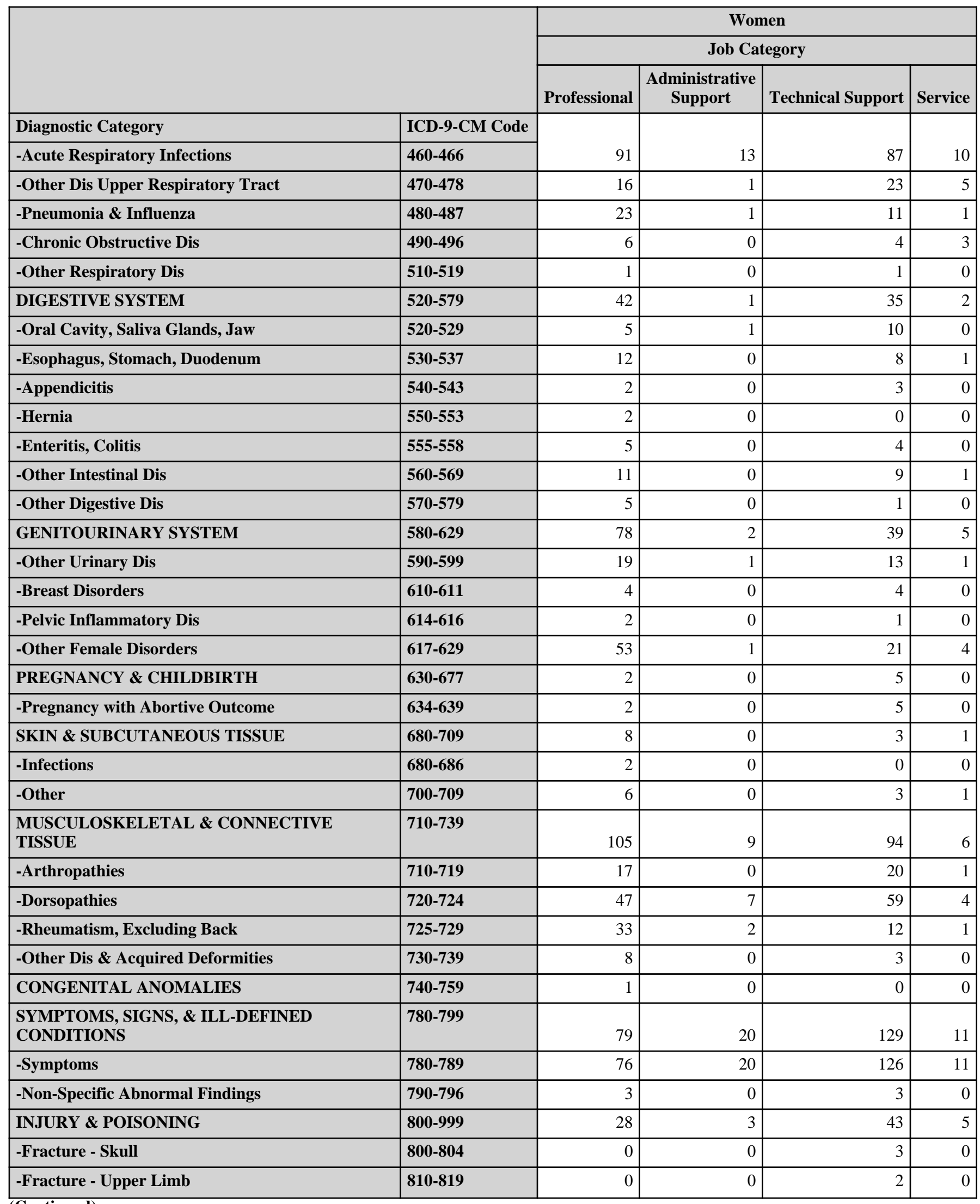

(Continued)

*Only those diagnostic categories and gender/job category combinations with at least one occurrence appear in this table. 


\section{Savannah River Site 2006}

\section{Absence Data}

Appendix I. Number of Diagnoses in Each Diagnostic Category by Gender and Job Category*

\begin{tabular}{|c|c|c|c|c|c|}
\hline & & \multicolumn{4}{|c|}{ Women } \\
\hline & & \multicolumn{3}{|c|}{ Job Category } & \multirow[b]{2}{*}{ TOTAL } \\
\hline & & Security and Fire & Crafts & Line Operators & \\
\hline Diagnostic Category & ICD-9-CM Code & \multirow[b]{2}{*}{0} & \multirow[b]{2}{*}{2} & \multirow[b]{2}{*}{55} & \multirow[b]{2}{*}{258} \\
\hline -Acute Respiratory Infections & $460-466$ & & & & \\
\hline -Other Dis Upper Respiratory Tract & $470-478$ & 0 & 0 & 24 & 69 \\
\hline -Pneumonia \& Influenza & $480-487$ & 0 & 0 & 6 & 42 \\
\hline -Chronic Obstructive Dis & $490-496$ & 0 & 0 & 4 & 17 \\
\hline -Other Respiratory Dis & $510-519$ & 0 & 0 & 0 & 2 \\
\hline DIGESTIVE SYSTEM & $520-579$ & 0 & 0 & 26 & 106 \\
\hline -Oral Cavity, Saliva Glands, Jaw & $520-529$ & 0 & 0 & 8 & 24 \\
\hline -Esophagus, Stomach, Duodenum & $530-537$ & 0 & 0 & 4 & 25 \\
\hline -Appendicitis & $540-543$ & 0 & 0 & 0 & 5 \\
\hline -Hernia & $550-553$ & 0 & 0 & 2 & 4 \\
\hline -Enteritis, Colitis & $555-558$ & 0 & 0 & 5 & 14 \\
\hline -Other Intestinal Dis & $560-569$ & 0 & 0 & 4 & 25 \\
\hline -Other Digestive Dis & $570-579$ & 0 & 0 & 3 & 9 \\
\hline GENITOURINARY SYSTEM & $580-629$ & 0 & 1 & 12 & 137 \\
\hline -Other Urinary Dis & $590-599$ & 0 & 0 & 7 & 41 \\
\hline -Breast Disorders & $610-611$ & 0 & 0 & 1 & 9 \\
\hline -Pelvic Inflammatory Dis & 614-616 & 0 & 0 & 1 & 4 \\
\hline -Other Female Disorders & $617-629$ & 0 & 1 & 3 & 83 \\
\hline PREGNANCY \& CHILDBIRTH & $630-677$ & 0 & 0 & 1 & 8 \\
\hline -Pregnancy with Abortive Outcome & 634-639 & 0 & 0 & 1 & 8 \\
\hline SKIN \& SUBCUTANEOUS TISSUE & 680-709 & 0 & 1 & 4 & 17 \\
\hline -Infections & $680-686$ & 0 & 0 & 1 & 3 \\
\hline -Other & 700-709 & 0 & 1 & 3 & 14 \\
\hline $\begin{array}{l}\text { MUSCULOSKELETAL \& CONNECTIVE } \\
\text { TISSUE }\end{array}$ & $710-739$ & 0 & 2 & 73 & 289 \\
\hline -Arthropathies & $710-719$ & 0 & 1 & 18 & 57 \\
\hline -Dorsopathies & $720-724$ & 0 & 1 & 38 & 156 \\
\hline -Rheumatism, Excluding Back & 725-729 & 0 & 0 & 15 & 63 \\
\hline -Other Dis \& Acquired Deformities & $730-739$ & 0 & 0 & 2 & 13 \\
\hline CONGENITAL ANOMALIES & $740-759$ & 0 & 0 & 0 & 1 \\
\hline $\begin{array}{l}\text { SYMPTOMS, SIGNS, \& ILL-DEFINED } \\
\text { CONDITIONS }\end{array}$ & $780-799$ & 0 & 2 & 62 & 303 \\
\hline -Symptoms & 780-789 & 0 & 2 & 60 & 295 \\
\hline -Non-Specific Abnormal Findings & $790-796$ & 0 & 0 & 2 & 8 \\
\hline INJURY \& POISONING & 800-999 & 0 & 1 & 20 & 100 \\
\hline -Fracture - Skull & $800-804$ & 0 & 0 & 0 & 3 \\
\hline -Fracture - Upper Limb & \begin{tabular}{|l|}
$810-819$ \\
\end{tabular} & 0 & 0 & 0 & 2 \\
\hline
\end{tabular}

(Continued)

*Only those diagnostic categories and gender/job category combinations with at least one occurrence appear in this table. 


\section{Savannah River Site 2006}

\section{Absence Data}

Appendix I. Number of Diagnoses in Each Diagnostic Category by Gender and Job Category*

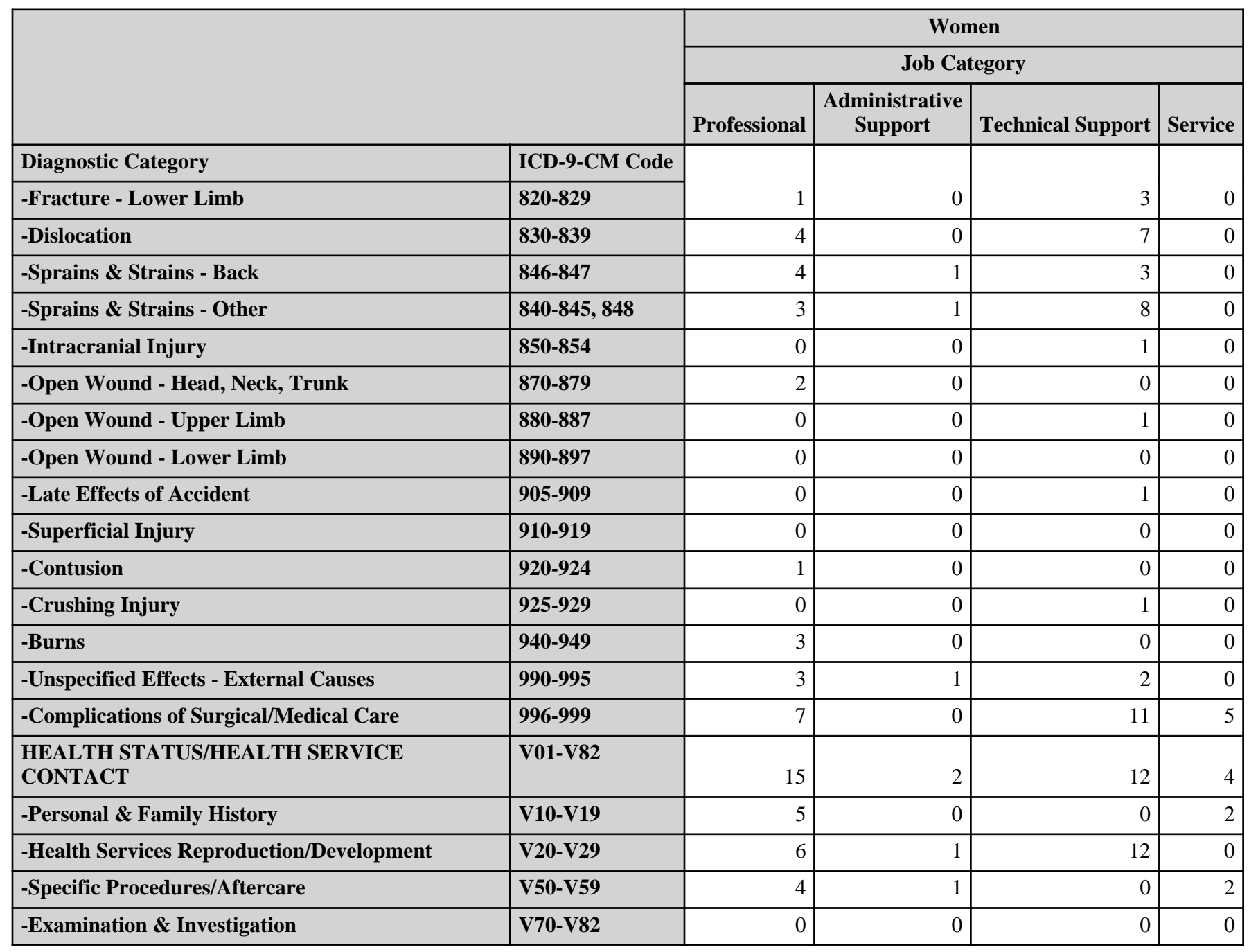

*Only those diagnostic categories and gender/job category combinations with at least one occurrence appear in this table. 


\section{Savannah River Site 2006}

\section{Absence Data}

Appendix I. Number of Diagnoses in Each Diagnostic Category by Gender and Job Category*

\begin{tabular}{|c|c|c|c|c|c|}
\hline & & \multicolumn{4}{|c|}{ Women } \\
\hline & & \multicolumn{3}{|c|}{ Job Category } & \multirow[b]{2}{*}{ TOTAL } \\
\hline & & Security and Fire & Crafts & Line Operators & \\
\hline Diagnostic Category & ICD-9-CM Code & \multirow[b]{2}{*}{0} & \multirow[b]{2}{*}{0} & \multirow[b]{2}{*}{1} & \multirow[b]{2}{*}{5} \\
\hline -Fracture - Lower Limb & $820-829$ & & & & \\
\hline -Dislocation & $830-839$ & 0 & 0 & 0 & 11 \\
\hline -Sprains \& Strains - Back & 846-847 & 0 & 0 & 4 & 12 \\
\hline -Sprains \& Strains - Other & $840-845,848$ & 0 & 1 & 2 & 15 \\
\hline -Intracranial Injury & $850-854$ & 0 & 0 & 0 & 1 \\
\hline -Open Wound - Head, Neck, Trunk & $870-879$ & 0 & 0 & 0 & 2 \\
\hline -Open Wound - Upper Limb & $880-887$ & 0 & 0 & 0 & 1 \\
\hline -Open Wound - Lower Limb & 890-897 & 0 & 0 & 1 & 1 \\
\hline -Late Effects of Accident & 905-909 & 0 & 0 & 0 & 1 \\
\hline -Superficial Injury & 910-919 & 0 & 0 & 1 & 1 \\
\hline -Contusion & $920-924$ & 0 & 0 & 0 & 1 \\
\hline -Crushing Injury & 925-929 & 0 & 0 & 0 & 1 \\
\hline -Burns & 940-949 & 0 & 0 & 3 & 6 \\
\hline -Unspecified Effects - External Causes & $990-995$ & 0 & 0 & 2 & 8 \\
\hline -Complications of Surgical/Medical Care & 996-999 & 0 & 0 & 6 & 29 \\
\hline $\begin{array}{l}\text { HEALTH STATUS/HEALTH SERVICE } \\
\text { CONTACT }\end{array}$ & V01-V82 & 0 & 1 & 7 & 41 \\
\hline -Personal \& Family History & V10-V19 & 0 & 1 & 0 & 8 \\
\hline -Health Services Reproduction/Development & V20-V29 & 0 & 0 & 4 & 23 \\
\hline -Specific Procedures/Aftercare & V50-V59 & 0 & 0 & 2 & 9 \\
\hline -Examination \& Investigation & V70-V82 & 0 & 0 & 1 & 1 \\
\hline
\end{tabular}

\begin{tabular}{|c|c|c|c|c|c|c|c|c|}
\hline & \multicolumn{8}{|c|}{ Women } \\
\hline & \multicolumn{7}{|c|}{ Job Category } & \multirow[b]{2}{*}{ TOTAL } \\
\hline & Professional & $\begin{array}{c}\text { Administrative } \\
\text { Support }\end{array}$ & $\begin{array}{l}\text { Technical } \\
\text { Support }\end{array}$ & Service & $\begin{array}{l}\text { Security and } \\
\text { Fire }\end{array}$ & Crafts & $\begin{array}{c}\text { Line } \\
\text { Operators }\end{array}$ & \\
\hline Diagnostic Category & \multirow[b]{2}{*}{645} & \multirow[b]{2}{*}{73} & \multirow[b]{2}{*}{588} & \multirow[b]{2}{*}{60} & \multirow[b]{2}{*}{1} & \multirow[b]{2}{*}{12} & \multirow[b]{2}{*}{371} & \multirow[b]{2}{*}{1,750} \\
\hline TOTAL & & & & & & & & \\
\hline
\end{tabular}

*Only those diagnostic categories and gender/job category combinations with at least one occurrence appear in this table. 


\section{Savannah River Site 2006}

\section{Absence Data}

Appendix I. Number of Diagnoses in Each Diagnostic Category by Gender and Job Category*

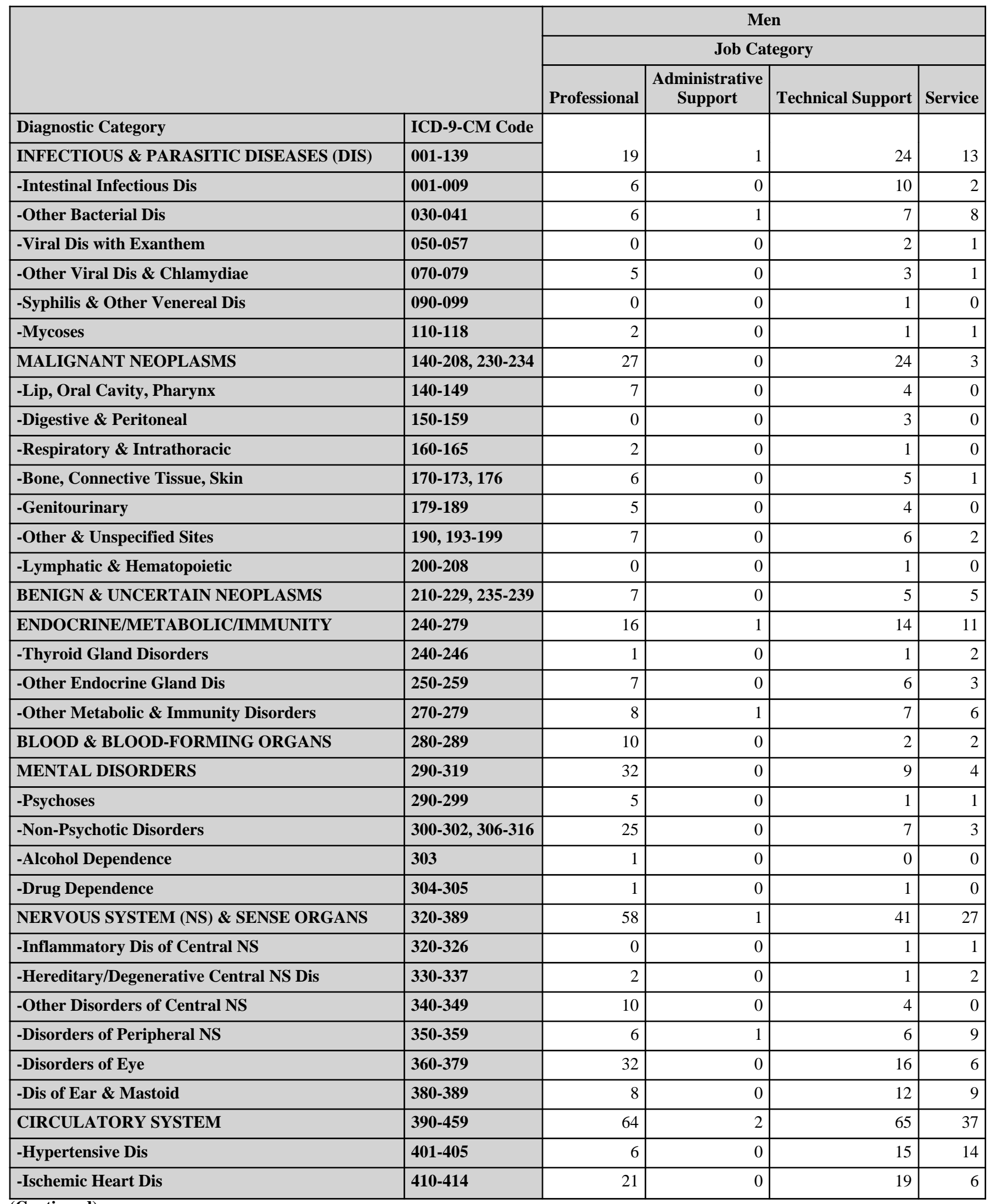

(Continued)

*Only those diagnostic categories and gender/job category combinations with at least one occurrence appear in this table. 


\section{Savannah River Site 2006}

\section{Absence Data}

Appendix I. Number of Diagnoses in Each Diagnostic Category by Gender and Job Category*

\begin{tabular}{|c|c|c|c|c|c|}
\hline & & \multicolumn{4}{|c|}{ Men } \\
\hline & & \multicolumn{3}{|c|}{ Job Category } & \multirow[b]{2}{*}{ TOTAL } \\
\hline & & Security and Fire & Crafts & Line Operators & \\
\hline Diagnostic Category & ICD-9-CM Code & \multirow[b]{2}{*}{3} & \multirow[b]{2}{*}{4} & \multirow[b]{2}{*}{22} & \multirow[b]{2}{*}{86} \\
\hline INFECTIOUS \& PARASITIC DISEASES (DIS) & 001-139 & & & & \\
\hline -Intestinal Infectious Dis & 001-009 & 1 & 1 & 10 & 30 \\
\hline -Other Bacterial Dis & 030-041 & 0 & 2 & 5 & 29 \\
\hline -Viral Dis with Exanthem & 050-057 & 1 & 1 & 2 & 7 \\
\hline -Other Viral Dis \& Chlamydiae & 070-079 & 1 & 0 & 5 & 15 \\
\hline -Syphilis \& Other Venereal Dis & \begin{tabular}{|c|}
$090-099$ \\
\end{tabular} & 0 & 0 & 0 & 1 \\
\hline -Mycoses & $110-118$ & 0 & 0 & 0 & 4 \\
\hline MALIGNANT NEOPLASMS & $140-208,230-234$ & 2 & 2 & 1 & 59 \\
\hline -Lip, Oral Cavity, Pharynx & $140-149$ & 0 & 0 & 0 & 11 \\
\hline -Digestive \& Peritoneal & $150-159$ & 0 & 1 & 0 & 4 \\
\hline -Respiratory \& Intrathoracic & $160-165$ & 0 & 0 & 0 & 3 \\
\hline -Bone, Connective Tissue, Skin & 170-173, 176 & 2 & 0 & 1 & 15 \\
\hline -Genitourinary & 179-189 & 0 & 1 & 0 & 10 \\
\hline -Other \& Unspecified Sites & 190, 193-199 & 0 & 0 & 0 & 15 \\
\hline -Lymphatic \& Hematopoietic & $200-208$ & 0 & 0 & 0 & 1 \\
\hline BENIGN \& UNCERTAIN NEOPLASMS & 210-229, 235-239 & 0 & 2 & 3 & 22 \\
\hline ENDOCRINE/METABOLIC/IMMUNITY & $240-279$ & 2 & 4 & 11 & 59 \\
\hline -Thyroid Gland Disorders & $240-246$ & 0 & 1 & 0 & 5 \\
\hline -Other Endocrine Gland Dis & $250-259$ & 0 & 2 & 2 & 20 \\
\hline -Other Metabolic \& Immunity Disorders & $270-279$ & 2 & 1 & 9 & 34 \\
\hline BLOOD \& BLOOD-FORMING ORGANS & $280-289$ & 0 & 0 & 3 & 17 \\
\hline MENTAL DISORDERS & 290-319 & 0 & 2 & 0 & 47 \\
\hline -Psychoses & $290-299$ & 0 & 0 & 0 & 7 \\
\hline -Non-Psychotic Disorders & $300-302,306-316$ & 0 & 2 & 0 & 37 \\
\hline -Alcohol Dependence & 303 & 0 & 0 & 0 & 1 \\
\hline -Drug Dependence & 304-305 & 0 & 0 & 0 & 2 \\
\hline NERVOUS SYSTEM (NS) \& SENSE ORGANS & 320-389 & 0 & 6 & 22 & 155 \\
\hline -Inflammatory Dis of Central NS & $320-326$ & 0 & 0 & 0 & 2 \\
\hline -Hereditary/Degenerative Central NS Dis & 330-337 & 0 & 0 & 0 & 5 \\
\hline -Other Disorders of Central NS & $340-349$ & 0 & 0 & 1 & 15 \\
\hline -Disorders of Peripheral NS & $350-359$ & 0 & 3 & 5 & 30 \\
\hline -Disorders of Eye & $360-379$ & 0 & 2 & 7 & 63 \\
\hline -Dis of Ear \& Mastoid & $380-389$ & 0 & 1 & 9 & 39 \\
\hline CIRCULATORY SYSTEM & $390-459$ & 4 & 10 & 20 & 202 \\
\hline -Hypertensive Dis & $401-405$ & 1 & 4 & 9 & 49 \\
\hline -Ischemic Heart Dis & $410-414$ & 3 & 3 & 3 & 55 \\
\hline
\end{tabular}

(Continued)

*Only those diagnostic categories and gender/job category combinations with at least one occurrence appear in this table. 


\section{Savannah River Site 2006}

\section{Absence Data}

Appendix I. Number of Diagnoses in Each Diagnostic Category by Gender and Job Category*

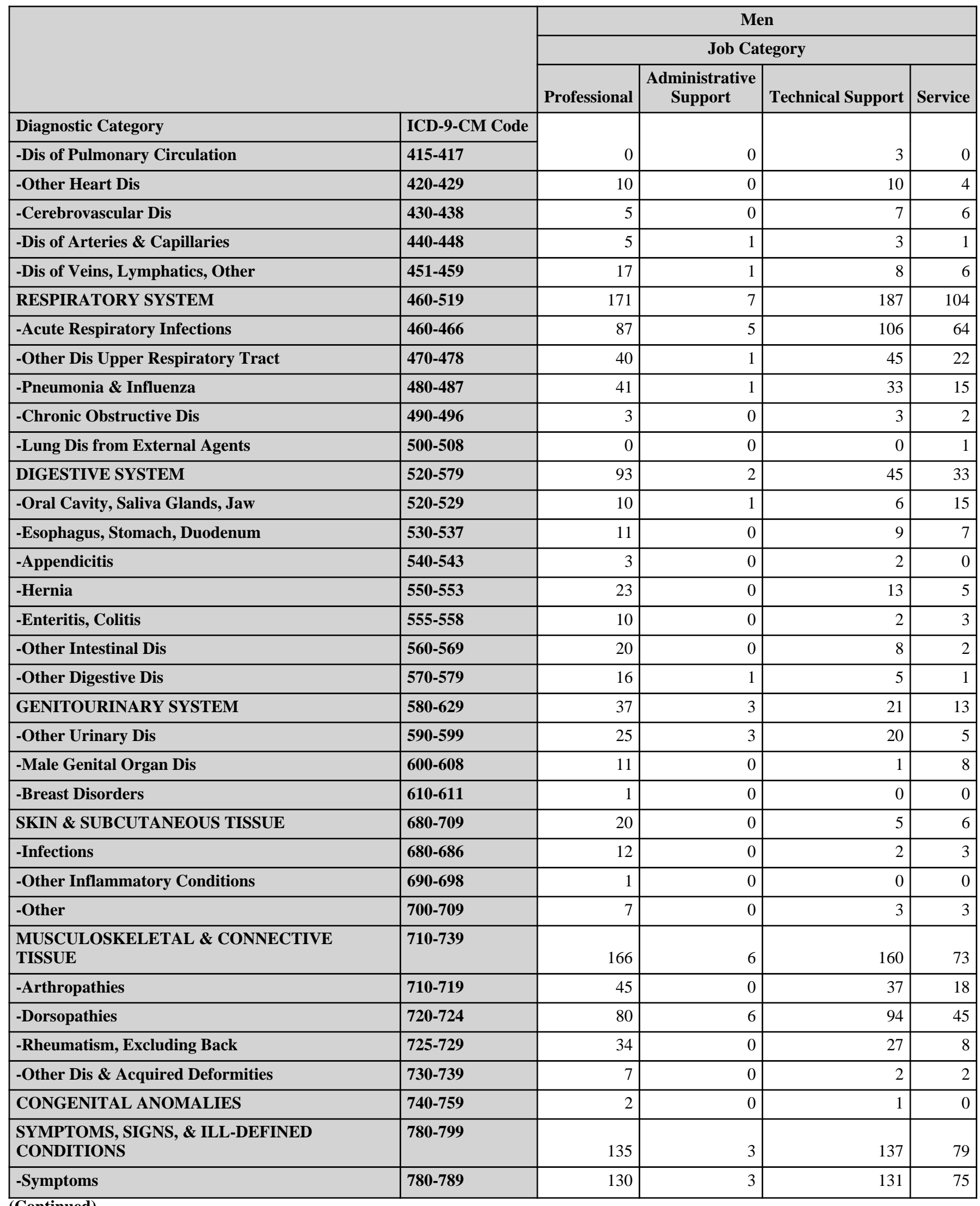

(Continued)

*Only those diagnostic categories and gender/job category combinations with at least one occurrence appear in this table. 


\section{Savannah River Site 2006}

\section{Absence Data}

Appendix I. Number of Diagnoses in Each Diagnostic Category by Gender and Job Category*

\begin{tabular}{|c|c|c|c|c|c|}
\hline & & \multicolumn{4}{|c|}{ Men } \\
\hline & & \multicolumn{3}{|c|}{ Job Category } & \multirow[b]{2}{*}{ TOTAL } \\
\hline & & Security and Fire & Crafts & Line Operators & \\
\hline Diagnostic Category & ICD-9-CM Code & \multirow[b]{2}{*}{0} & \multirow[b]{2}{*}{0} & \multirow[b]{2}{*}{0} & \multirow[b]{2}{*}{3} \\
\hline -Dis of Pulmonary Circulation & $415-417$ & & & & \\
\hline -Other Heart Dis & $420-429$ & 0 & 3 & 1 & 28 \\
\hline -Cerebrovascular Dis & $430-438$ & 0 & 0 & 0 & 18 \\
\hline -Dis of Arteries \& Capillaries & $440-448$ & 0 & 0 & 0 & 10 \\
\hline -Dis of Veins, Lymphatics, Other & $451-459$ & 0 & 0 & 7 & 39 \\
\hline RESPIRATORY SYSTEM & $460-519$ & 5 & 12 & 124 & 610 \\
\hline -Acute Respiratory Infections & $460-466$ & 2 & 8 & 81 & 353 \\
\hline -Other Dis Upper Respiratory Tract & $470-478$ & 1 & 2 & 31 & 142 \\
\hline -Pneumonia \& Influenza & $480-487$ & 2 & 2 & 11 & 105 \\
\hline -Chronic Obstructive Dis & $490-496$ & 0 & 0 & 1 & 9 \\
\hline -Lung Dis from External Agents & $500-508$ & 0 & 0 & 0 & 1 \\
\hline DIGESTIVE SYSTEM & $520-579$ & 1 & 7 & 42 & 223 \\
\hline -Oral Cavity, Saliva Glands, Jaw & $520-529$ & 0 & 1 & 12 & 45 \\
\hline -Esophagus, Stomach, Duodenum & $530-537$ & 1 & 1 & 12 & 41 \\
\hline -Appendicitis & $540-543$ & 0 & 0 & 0 & 5 \\
\hline -Hernia & $550-553$ & 0 & 4 & 9 & 54 \\
\hline -Enteritis, Colitis & $555-558$ & 0 & 0 & 1 & 16 \\
\hline -Other Intestinal Dis & $560-569$ & 0 & 0 & 4 & 34 \\
\hline -Other Digestive Dis & $570-579$ & 0 & 1 & 4 & 28 \\
\hline GENITOURINARY SYSTEM & $580-629$ & 1 & 2 & 16 & 93 \\
\hline -Other Urinary Dis & $590-599$ & 0 & 2 & 13 & 68 \\
\hline -Male Genital Organ Dis & $600-608$ & 1 & 0 & 3 & 24 \\
\hline -Breast Disorders & $610-611$ & 0 & 0 & 0 & 1 \\
\hline SKIN \& SUBCUTANEOUS TISSUE & $680-709$ & 1 & 0 & 3 & 35 \\
\hline -Infections & $680-686$ & 0 & 0 & 2 & 19 \\
\hline -Other Inflammatory Conditions & $690-698$ & 0 & 0 & 0 & 1 \\
\hline -Other & 700-709 & 1 & 0 & 1 & 15 \\
\hline $\begin{array}{l}\text { MUSCULOSKELETAL \& CONNECTIVE } \\
\text { TISSUE }\end{array}$ & $710-739$ & 4 & 12 & 94 & 515 \\
\hline -Arthropathies & $710-719$ & 0 & 4 & 15 & 119 \\
\hline -Dorsopathies & $720-724$ & 1 & 6 & 62 & 294 \\
\hline -Rheumatism, Excluding Back & $725-729$ & 3 & 2 & 16 & 90 \\
\hline -Other Dis \& Acquired Deformities & $730-739$ & 0 & 0 & 1 & 12 \\
\hline CONGENITAL ANOMALIES & 740-759 & 0 & 0 & 0 & 3 \\
\hline $\begin{array}{l}\text { SYMPTOMS, SIGNS, \& ILL-DEFINED } \\
\text { CONDITIONS }\end{array}$ & $780-799$ & 5 & 4 & 101 & 464 \\
\hline -Symptoms & $780-789$ & 5 & 4 & 97 & 445 \\
\hline
\end{tabular}

(Continued)

*Only those diagnostic categories and gender/job category combinations with at least one occurrence appear in this table. 


\section{Savannah River Site 2006}

\section{Absence Data}

Appendix I. Number of Diagnoses in Each Diagnostic Category by Gender and Job Category*

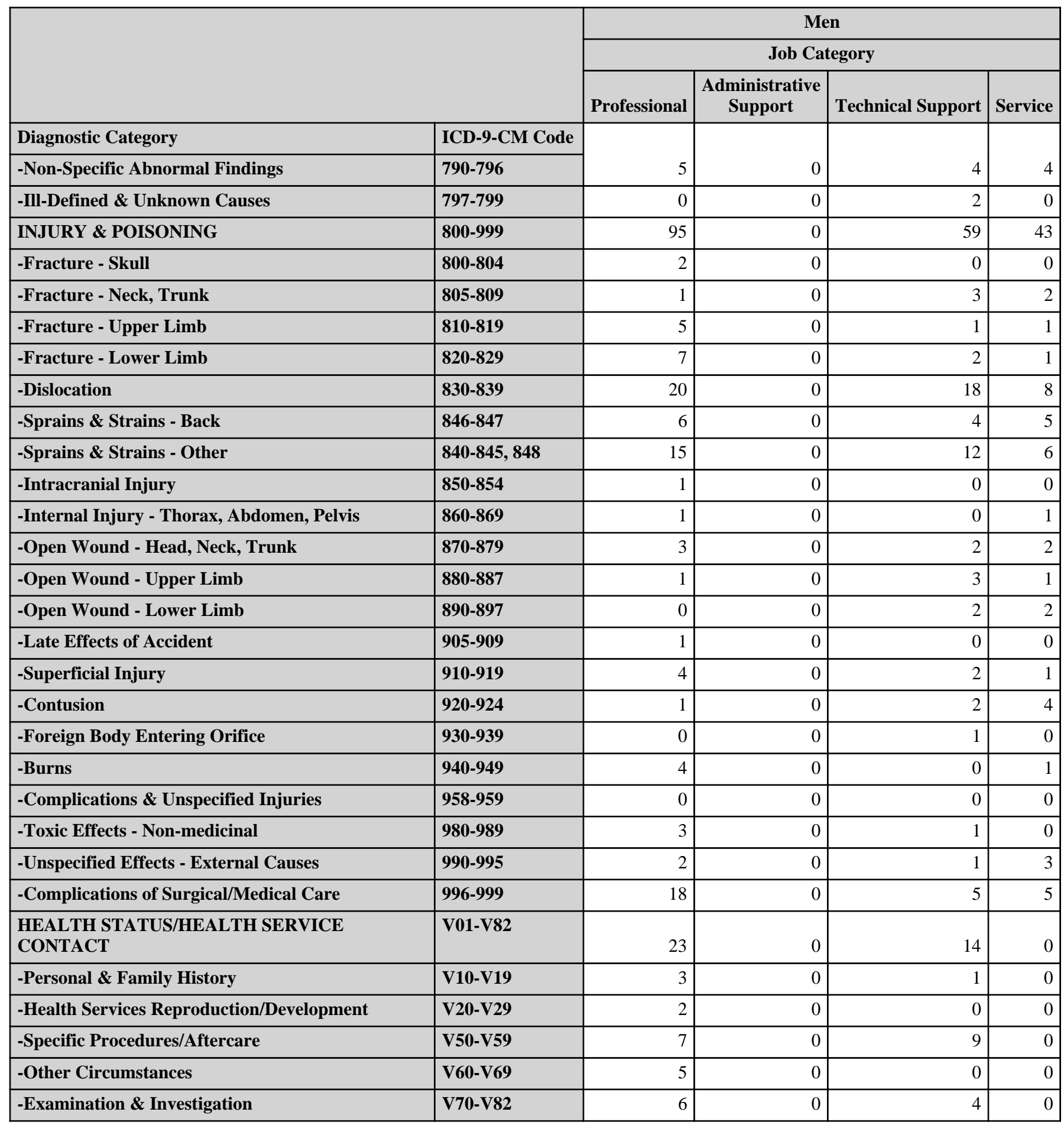

*Only those diagnostic categories and gender/job category combinations with at least one occurrence appear in this table. 


\section{Savannah River Site 2006}

\section{Absence Data}

Appendix I. Number of Diagnoses in Each Diagnostic Category by Gender and Job Category*

\begin{tabular}{|c|c|c|c|c|c|}
\hline & & \multicolumn{4}{|c|}{ Men } \\
\hline & & \multicolumn{3}{|c|}{ Job Category } & \multirow[b]{2}{*}{ TOTAL } \\
\hline & & Security and Fire & Crafts & Line Operators & \\
\hline Diagnostic Category & ICD-9-CM Code & \multirow[b]{2}{*}{0} & \multirow[b]{2}{*}{0} & \multirow[b]{2}{*}{4} & \multirow[b]{2}{*}{17} \\
\hline -Non-Specific Abnormal Findings & $790-796$ & & & & \\
\hline -Ill-Defined \& Unknown Causes & 797-799 & 0 & 0 & 0 & 2 \\
\hline INJURY \& POISONING & $800-999$ & 0 & 15 & 64 & 276 \\
\hline -Fracture - Skull & $800-804$ & 0 & 0 & 4 & 6 \\
\hline -Fracture - Neck, Trunk & 805-809 & 0 & 0 & 2 & 8 \\
\hline -Fracture - Upper Limb & 810-819 & 0 & 0 & 2 & 9 \\
\hline -Fracture - Lower Limb & $820-829$ & 0 & 0 & 1 & 11 \\
\hline -Dislocation & $830-839$ & 0 & 1 & 4 & 51 \\
\hline -Sprains \& Strains - Back & 846-847 & 0 & 4 & 4 & 23 \\
\hline -Sprains \& Strains - Other & $840-845,848$ & 0 & 3 & 15 & 51 \\
\hline -Intracranial Injury & $850-854$ & 0 & 0 & 1 & 2 \\
\hline -Internal Injury - Thorax, Abdomen, Pelvis & $860-869$ & 0 & 0 & 0 & 2 \\
\hline -Open Wound - Head, Neck, Trunk & 870-879 & 0 & 1 & 2 & 10 \\
\hline -Open Wound - Upper Limb & 880-887 & 0 & 1 & 4 & 10 \\
\hline -Open Wound - Lower Limb & $890-897$ & 0 & 0 & 1 & 5 \\
\hline -Late Effects of Accident & $905-909$ & 0 & 0 & 0 & 1 \\
\hline -Superficial Injury & 910-919 & 0 & 1 & 1 & 9 \\
\hline -Contusion & 920-924 & 0 & 4 & 5 & 16 \\
\hline -Foreign Body Entering Orifice & 930-939 & 0 & 0 & 1 & 2 \\
\hline -Burns & 940-949 & 0 & 0 & 0 & 5 \\
\hline -Complications \& Unspecified Injuries & 958-959 & 0 & 0 & 1 & 1 \\
\hline -Toxic Effects - Non-medicinal & 980-989 & 0 & 0 & 0 & 4 \\
\hline -Unspecified Effects - External Causes & $990-995$ & 0 & 0 & 10 & 16 \\
\hline -Complications of Surgical/Medical Care & 996-999 & 0 & 0 & 6 & 34 \\
\hline $\begin{array}{l}\text { HEALTH STATUS/HEALTH SERVICE } \\
\text { CONTACT }\end{array}$ & V01-V82 & 0 & 1 & 4 & 42 \\
\hline -Personal \& Family History & V10-V19 & 0 & 0 & 0 & 4 \\
\hline -Health Services Reproduction/Development & V20-V29 & 0 & 0 & 0 & 2 \\
\hline -Specific Procedures/Aftercare & V50-V59 & 0 & 1 & 4 & 21 \\
\hline -Other Circumstances & V60-V69 & 0 & 0 & 0 & 5 \\
\hline -Examination \& Investigation & V70-V82 & 0 & 0 & 0 & 10 \\
\hline
\end{tabular}

*Only those diagnostic categories and gender/job category combinations with at least one occurrence appear in this table. 
Savannah River Site 2006

Absence Data

Appendix I. Number of Diagnoses in Each Diagnostic Category by Gender and Job Category*

\begin{tabular}{|c|c|c|c|c|c|c|c|c|}
\hline & \multicolumn{8}{|c|}{ Men } \\
\hline & \multicolumn{7}{|c|}{ Job Category } & \multirow[b]{2}{*}{ TOTAL } \\
\hline & Professional & $\begin{array}{c}\text { Administrative } \\
\text { Support }\end{array}$ & $\begin{array}{l}\text { Technical } \\
\text { Support }\end{array}$ & Service & $\begin{array}{c}\text { Security and } \\
\text { Fire }\end{array}$ & Crafts & $\begin{array}{c}\text { Line } \\
\text { Operators }\end{array}$ & \\
\hline Diagnostic Category & \multirow[b]{2}{*}{975} & \multirow[b]{2}{*}{26} & \multirow[b]{2}{*}{813} & \multirow[b]{2}{*}{453} & \multirow[b]{2}{*}{28} & \multirow[b]{2}{*}{83} & \multirow[b]{2}{*}{530} & \multirow[b]{2}{*}{2,908} \\
\hline TOTAL & & & & & & & & \\
\hline
\end{tabular}

*Only those diagnostic categories and gender/job category combinations with at least one occurrence appear in this table. 


\section{Savannah River Site 2006}

\section{Absence Data}

Appendix J. Total Number of Calendar Days Absent in Each Diagnostic Category by Gender and Job Category*

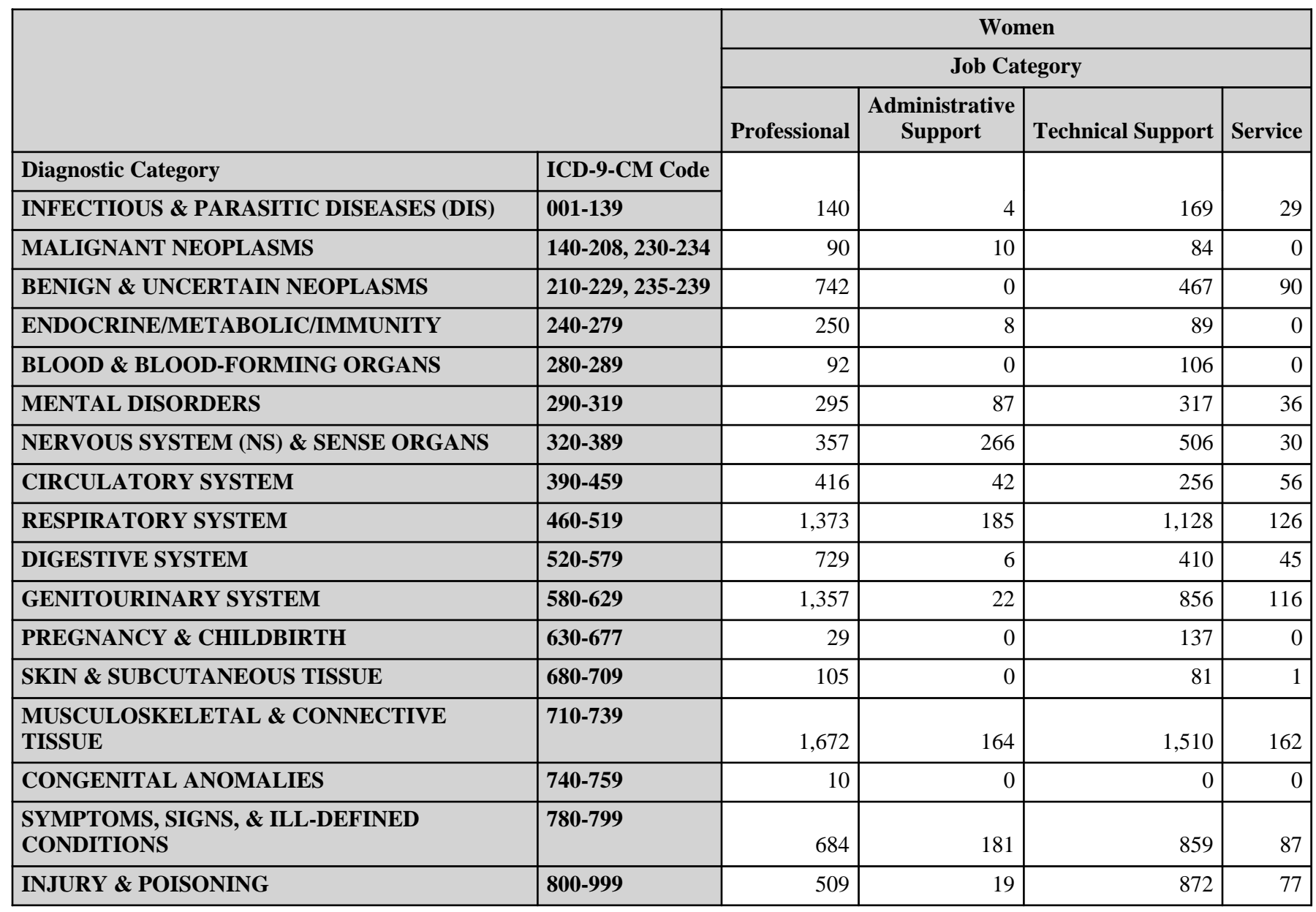

\footnotetext{
*Absences with >1 ICD-9-CM code in the same diagnostic category were counted only once. Only those diagnostic categories and gender/job category combinations with at least one occurrence appear in this table.
} 


\section{Savannah River Site 2006}

\section{Absence Data}

Appendix J. Total Number of Calendar Days Absent in Each Diagnostic Category by Gender and Job Category*

\begin{tabular}{|c|c|c|c|c|c|}
\hline & & \multicolumn{4}{|c|}{ Women } \\
\hline & & \multicolumn{3}{|c|}{ Job Category } & \multirow[b]{2}{*}{ TOTAL } \\
\hline & & Security and Fire & Crafts & Line Operators & \\
\hline Diagnostic Category & ICD-9-CM Code & \multirow[b]{2}{*}{0} & \multirow[b]{2}{*}{0} & \multirow[b]{2}{*}{181} & \multirow[b]{2}{*}{523} \\
\hline INFECTIOUS \& PARASITIC DISEASES (DIS) & 001-139 & & & & \\
\hline MALIGNANT NEOPLASMS & $140-208,230-234$ & 0 & 14 & 38 & 236 \\
\hline BENIGN \& UNCERTAIN NEOPLASMS & 210-229, 235-239 & 73 & 43 & 166 & 1,581 \\
\hline ENDOCRINE/METABOLIC/IMMUNITY & $240-279$ & 0 & 0 & 292 & 639 \\
\hline BLOOD \& BLOOD-FORMING ORGANS & $280-289$ & 0 & 0 & 0 & 198 \\
\hline MENTAL DISORDERS & 290-319 & 0 & 0 & 296 & 1,031 \\
\hline NERVOUS SYSTEM (NS) \& SENSE ORGANS & $320-389$ & 0 & 0 & 479 & 1,638 \\
\hline CIRCULATORY SYSTEM & $390-459$ & 0 & 0 & 366 & 1,136 \\
\hline RESPIRATORY SYSTEM & $460-519$ & 0 & 14 & 992 & 3,818 \\
\hline DIGESTIVE SYSTEM & $520-579$ & 0 & 0 & 446 & 1,636 \\
\hline GENITOURINARY SYSTEM & $580-629$ & 0 & 55 & 274 & 2,680 \\
\hline PREGNANCY \& CHILDBIRTH & $630-677$ & 0 & 0 & 5 & 171 \\
\hline SKIN \& SUBCUTANEOUS TISSUE & $680-709$ & 0 & 8 & 54 & 249 \\
\hline $\begin{array}{l}\text { MUSCULOSKELETAL \& CONNECTIVE } \\
\text { TISSUE }\end{array}$ & $710-739$ & 0 & 49 & 843 & 4,400 \\
\hline CONGENITAL ANOMALIES & 740-759 & 0 & 0 & 0 & 10 \\
\hline $\begin{array}{l}\text { SYMPTOMS, SIGNS, \& ILL-DEFINED } \\
\text { CONDITIONS }\end{array}$ & $780-799$ & 0 & 22 & 303 & 2,136 \\
\hline INJURY \& POISONING & 800-999 & 0 & 5 & 365 & 1,847 \\
\hline
\end{tabular}

\footnotetext{
*Absences with >1 ICD-9-CM code in the same diagnostic category were counted only once. Only those diagnostic categories and gender/job category combinations with at least one occurrence appear in this table.
} 


\section{Savannah River Site 2006}

\section{Absence Data}

Appendix J. Total Number of Calendar Days Absent in Each Diagnostic Category by Gender and Job Category*

\begin{tabular}{|c|c|c|c|c|c|}
\hline & & \multicolumn{4}{|c|}{ Men } \\
\hline & & \multicolumn{4}{|c|}{ Job Category } \\
\hline & & Professional & $\begin{array}{l}\text { Administrative } \\
\text { Support }\end{array}$ & Technical Support & Service \\
\hline Diagnostic Category & ICD-9-CM Code & \multirow[b]{2}{*}{150} & \multirow[b]{2}{*}{7} & \multirow[b]{2}{*}{257} & \multirow[b]{2}{*}{111} \\
\hline INFECTIOUS \& PARASITIC DISEASES (DIS) & 001-139 & & & & \\
\hline BENIGN \& UNCERTAIN NEOPLASMS & 210-229, 235-239 & 75 & 0 & 63 & 27 \\
\hline ENDOCRINE/METABOLIC/IMMUNITY & $240-279$ & 270 & 4 & 743 & 682 \\
\hline BLOOD \& BLOOD-FORMING ORGANS & $280-289$ & 70 & 0 & 33 & 201 \\
\hline MENTAL DISORDERS & 290-319 & 635 & 0 & 300 & 294 \\
\hline DIGESTIVE SYSTEM & $520-579$ & 1,629 & 30 & 734 & 510 \\
\hline GENITOURINARY SYSTEM & $580-629$ & 350 & 6 & 312 & 115 \\
\hline SKIN \& SUBCUTANEOUS TISSUE & $680-709$ & 444 & 0 & 35 & 85 \\
\hline $\begin{array}{l}\text { MUSCULOSKELETAL \& CONNECTIVE } \\
\text { TISSUE }\end{array}$ & $710-739$ & 1,985 & 86 & 1,801 & 1,386 \\
\hline CONGENITAL ANOMALIES & $740-759$ & 9 & 0 & 5 & 0 \\
\hline $\begin{array}{l}\text { SYMPTOMS, SIGNS, \& ILL-DEFINED } \\
\text { CONDITIONS }\end{array}$ & 780-799 & 1,316 & 14 & 1,542 & 1,234 \\
\hline INJURY \& POISONING & $800-999$ & 1,517 & 0 & 884 & 881 \\
\hline
\end{tabular}

\footnotetext{
*Absences with >1 ICD-9-CM code in the same diagnostic category were counted only once. Only those diagnostic categories and gender/job category combinations with at least one occurrence appear in this table.
} 


\section{Savannah River Site 2006}

\section{Absence Data}

Appendix J. Total Number of Calendar Days Absent in Each Diagnostic Category by Gender and Job Category*

\begin{tabular}{|c|c|c|c|c|c|}
\hline & & \multicolumn{4}{|c|}{ Men } \\
\hline & & \multicolumn{3}{|c|}{ Job Category } & \multirow[b]{2}{*}{ TOTAL } \\
\hline & & Security and Fire & Crafts & Line Operators & \\
\hline Diagnostic Category & ICD-9-CM Code & \multirow[b]{2}{*}{39} & \multirow[b]{2}{*}{11} & \multirow[b]{2}{*}{222} & \multirow[b]{2}{*}{797} \\
\hline INFECTIOUS \& PARASITIC DISEASES (DIS) & 001-139 & & & & \\
\hline MALIGNANT NEOPLASMS & $140-208,230-234$ & 12 & 184 & 266 & 1,823 \\
\hline BENIGN \& UNCERTAIN NEOPLASMS & 210-229, 235-239 & 0 & 72 & 250 & 487 \\
\hline ENDOCRINE/METABOLIC/IMMUNITY & $240-279$ & 11 & 30 & 238 & 1,978 \\
\hline BLOOD \& BLOOD-FORMING ORGANS & $280-289$ & 0 & 0 & 41 & 345 \\
\hline MENTAL DISORDERS & 290-319 & 0 & 42 & 0 & 1,271 \\
\hline NERVOUS SYSTEM (NS) \& SENSE ORGANS & $320-389$ & 0 & 206 & 347 & 3,101 \\
\hline CIRCULATORY SYSTEM & $390-459$ & 35 & 169 & 263 & 4,699 \\
\hline RESPIRATORY SYSTEM & 460-519 & 33 & 87 & 1,072 & 5,163 \\
\hline DIGESTIVE SYSTEM & $520-579$ & 7 & 108 & 469 & 3,487 \\
\hline GENITOURINARY SYSTEM & $580-629$ & 7 & 6 & 255 & 1,051 \\
\hline SKIN \& SUBCUTANEOUS TISSUE & 680-709 & 4 & 0 & 44 & 612 \\
\hline $\begin{array}{l}\text { MUSCULOSKELETAL \& CONNECTIVE } \\
\text { TISSUE }\end{array}$ & $710-739$ & 19 & 289 & 1,234 & 6,800 \\
\hline CONGENITAL ANOMALIES & 740-759 & 0 & 0 & 0 & 14 \\
\hline $\begin{array}{l}\text { SYMPTOMS, SIGNS, \& ILL-DEFINED } \\
\text { CONDITIONS }\end{array}$ & \begin{tabular}{|l|}
$780-799$ \\
\end{tabular} & 12 & 19 & 777 & 4,914 \\
\hline INJURY \& POISONING & 800-999 & 0 & 391 & 1,221 & 4,894 \\
\hline
\end{tabular}

\footnotetext{
*Absences with >1 ICD-9-CM code in the same diagnostic category were counted only once. Only those diagnostic categories and gender/job category combinations with at least one occurrence appear in this table.
} 


\section{Savannah River Site 2006}

\section{Absence Data}

Appendix K. Age-Adjusted IIIness and Injury Rates by Diagnostic Category*

Part 1. Men

\begin{tabular}{|c|c|c|c|c|c|}
\hline & & $\begin{array}{c}\text { Number of } \\
\text { Diagnoses }\end{array}$ & $\begin{array}{c}\text { Age-Adjusted } \\
\text { Rate per 1,000** }\end{array}$ & $\begin{array}{c}\text { Lower } 95 \% \\
\text { Confidence } \\
\text { Limit per 1,000 }\end{array}$ & $\begin{array}{c}\text { Upper 95\% } \\
\text { Confidence } \\
\text { Limit per 1,000 }\end{array}$ \\
\hline Diagnostic Category & ICD-9-CM Code & & & & \\
\hline INFECTIOUS \& PARASITIC DISEASES (DIS) & 001-139 & 86 & 11.7 & 7.9 & 17.3 \\
\hline -Intestinal Infectious Dis & 001-009 & 30 & 2.9 & 1.8 & 4.7 \\
\hline -Other Bacterial Dis & 030-041 & 29 & 4.9 & 2.6 & 9.4 \\
\hline -Viral Dis with Exanthem & 050-057 & 7 & 1.8 & 0.4 & 7.8 \\
\hline -Other Viral Dis \& Chlamydiae & 070-079 & 15 & 1.3 & 0.7 & 2.6 \\
\hline -Syphilis \& Other Venereal Dis & 090-099 & 1 & 0.1 & 0.0 & 0.4 \\
\hline -Mycoses & $110-118$ & 4 & 0.7 & 0.2 & 2.4 \\
\hline MALIGNANT NEOPLASMS & $140-208,230-234$ & 59 & 5.0 & 3.7 & 6.8 \\
\hline -Lip, Oral Cavity, Pharynx & $140-149$ & 11 & 0.6 & 0.3 & 1.1 \\
\hline -Digestive \& Peritoneal & 150-159 & 4 & 0.5 & 0.2 & 1.5 \\
\hline -Respiratory \& Intrathoracic & $160-165$ & 3 & 0.5 & 0.1 & 1.6 \\
\hline -Bone, Connective Tissue, Skin & $170-173,176$ & 15 & 1.1 & 0.7 & 2.0 \\
\hline -Genitourinary & $179-189$ & 10 & 1.0 & 0.5 & 2.1 \\
\hline -Other \& Unspecified Sites & $190,193-199$ & 15 & 1.2 & 0.7 & 2.2 \\
\hline -Lymphatic \& Hematopoietic & 200-208 & 1 & 0.1 & 0.0 & 0.4 \\
\hline BENIGN \& UNCERTAIN NEOPLASMS & 210-229, 235-239 & 22 & 2.2 & 1.2 & 3.9 \\
\hline ENDOCRINE/METABOLIC/IMMUNITY & 240-279 & 59 & 7.2 & 4.2 & 12.5 \\
\hline -Thyroid Gland Disorders & $240-246$ & 5 & 0.3 & 0.1 & 0.8 \\
\hline -Other Endocrine Gland Dis & $250-259$ & 20 & 4.1 & 1.6 & 10.3 \\
\hline -Other Metabolic \& Immunity Disorders & 270-279 & 34 & 2.8 & 1.9 & 4.1 \\
\hline BLOOD \& BLOOD-FORMING ORGANS & 280-289 & 17 & 1.1 & 0.7 & 1.8 \\
\hline MENTAL DISORDERS & $290-319$ & 47 & 7.3 & 4.2 & 12.9 \\
\hline -Psychoses & 290-299 & 7 & 0.9 & 0.3 & 2.4 \\
\hline -Non-Psychotic Disorders & $300-302,306-316$ & 37 & 6.1 & 3.1 & 11.7 \\
\hline -Alcohol Dependence & 303 & 1 & 0.1 & 0.0 & 0.6 \\
\hline -Drug Dependence & $304-305$ & 2 & 0.3 & 0.1 & 1.2 \\
\hline NERVOUS SYSTEM (NS) \& SENSE ORGANS & 320-389 & 155 & 15.1 & 12.4 & 18.3 \\
\hline -Inflammatory Dis of Central NS & $320-326$ & 2 & 0.1 & 0.0 & 0.5 \\
\hline -Hereditary/Degenerative Central NS Dis & 330-337 & 5 & 0.3 & 0.1 & 0.7 \\
\hline -Other Disorders of Central NS & 340-349 & 15 & 1.4 & 0.7 & 2.7 \\
\hline -Disorders of Peripheral NS & 350-359 & 30 & 3.1 & 2.0 & 5.0 \\
\hline -Disorders of Eye & $360-379$ & 63 & 6.6 & 4.9 & 8.8 \\
\hline -Dis of Ear \& Mastoid & 380-389 & 39 & 3.4 & 2.3 & 5.1 \\
\hline CIRCULATORY SYSTEM & $390-459$ & 202 & 20.2 & 16.8 & 24.2 \\
\hline -Hypertensive Dis & $401-405$ & 49 & 6.7 & 4.6 & 9.8 \\
\hline
\end{tabular}

(Continued)

*Only those diagnostic categories with at least one occurrence appear in this table.

**Standardized to age distribution of 2000 U.S. population. 


\section{Savannah River Site 2006}

\section{Absence Data}

Appendix K. Age-Adjusted IIIness and Injury Rates by Diagnostic Category*

Part 1. Men

\begin{tabular}{|c|c|c|c|c|c|}
\hline & & $\begin{array}{l}\text { Number of } \\
\text { Diagnoses }\end{array}$ & $\begin{array}{c}\text { Age-Adjusted } \\
\text { Rate per } 1,000 * *\end{array}$ & $\begin{array}{c}\text { Lower } 95 \% \\
\text { Confidence } \\
\text { Limit per 1,000 }\end{array}$ & $\begin{array}{c}\text { Upper 95\% } \\
\text { Confidence } \\
\text { Limit per 1,000 }\end{array}$ \\
\hline Diagnostic Category & ICD-9-CM Code & \multirow[b]{2}{*}{55} & \multirow[b]{2}{*}{5.0} & \multirow[b]{2}{*}{3.6} & \multirow[b]{2}{*}{7.2} \\
\hline -Ischemic Heart Dis & $410-414$ & & & & \\
\hline -Dis of Pulmonary Circulation & $415-417$ & 3 & 0.2 & 0.1 & 0.5 \\
\hline -Other Heart Dis & $420-429$ & 28 & 2.3 & 1.5 & 3.4 \\
\hline -Cerebrovascular Dis & $430-438$ & 18 & 1.5 & 0.9 & 2.5 \\
\hline -Dis of Arteries \& Capillaries & $440-448$ & 10 & 0.7 & 0.4 & 1.5 \\
\hline -Dis of Veins, Lymphatics, Other & $451-459$ & 39 & 3.7 & 2.4 & 5.7 \\
\hline RESPIRATORY SYSTEM & $460-519$ & 610 & 78.8 & 69.2 & 89.6 \\
\hline -Acute Respiratory Infections & $460-466$ & 353 & 44.2 & 37.5 & 52.0 \\
\hline -Other Dis Upper Respiratory Tract & $470-478$ & 142 & 19.5 & 15.3 & 24.9 \\
\hline -Pneumonia \& Influenza & $480-487$ & 105 & 14.4 & 10.0 & 20.7 \\
\hline -Chronic Obstructive Dis & $490-496$ & 9 & 0.6 & 0.3 & 1.1 \\
\hline -Lung Dis from External Agents & $500-508$ & 1 & 0.1 & 0.0 & 0.4 \\
\hline DIGESTIVE SYSTEM & $520-579$ & 223 & 20.6 & 16.8 & 25.2 \\
\hline -Oral Cavity, Saliva Glands, Jaw & $520-529$ & 45 & 3.9 & 2.7 & 5.9 \\
\hline -Esophagus, Stomach, Duodenum & 530-537 & 41 & 3.0 & 2.2 & 4.2 \\
\hline -Appendicitis & $540-543$ & 5 & 0.6 & 0.2 & 2.2 \\
\hline -Hernia & $550-553$ & 54 & 5.8 & 3.4 & 9.8 \\
\hline -Enteritis, Colitis & $555-558$ & 16 & 1.4 & 0.8 & 2.4 \\
\hline -Other Intestinal Dis & $560-569$ & 34 & 2.9 & 2.0 & 4.4 \\
\hline -Other Digestive Dis & $570-579$ & 28 & 2.8 & 1.7 & 4.6 \\
\hline GENITOURINARY SYSTEM & $580-629$ & 93 & 14.2 & 9.8 & 20.7 \\
\hline -Other Urinary Dis & $590-599$ & 68 & 11.6 & 7.4 & 18.1 \\
\hline -Male Genital Organ Dis & $600-608$ & 24 & 2.6 & 1.6 & 4.2 \\
\hline -Breast Disorders & $610-611$ & 1 & 0.1 & 0.0 & 0.4 \\
\hline SKIN \& SUBCUTANEOUS TISSUE & $680-709$ & 35 & 4.0 & 2.6 & 6.1 \\
\hline -Infections & $680-686$ & 19 & 1.6 & 0.9 & 2.7 \\
\hline -Other Inflammatory Conditions & $690-698$ & 1 & 0.1 & 0.0 & 0.6 \\
\hline -Other & \begin{tabular}{|l|}
$700-709$ \\
\end{tabular} & 15 & 2.3 & 1.2 & 4.4 \\
\hline $\begin{array}{l}\text { MUSCULOSKELETAL \& CONNECTIVE } \\
\text { TISSUE }\end{array}$ & $710-739$ & 515 & 56.7 & 49.4 & 65.0 \\
\hline -Arthropathies & $710-719$ & 119 & 16.0 & 11.9 & 21.6 \\
\hline -Dorsopathies & $720-724$ & 294 & 30.7 & 26.0 & 36.2 \\
\hline -Rheumatism, Excluding Back & $725-729$ & 90 & 8.8 & 6.1 & 12.7 \\
\hline -Other Dis \& Acquired Deformities & \begin{tabular}{|l|}
$730-739$ \\
\end{tabular} & 12 & 1.2 & 0.5 & 2.5 \\
\hline CONGENITAL ANOMALIES & $740-759$ & 3 & 0.2 & 0.1 & 0.7 \\
\hline
\end{tabular}

(Continued)

*Only those diagnostic categories with at least one occurrence appear in this table.

**Standardized to age distribution of 2000 U.S. population. 


\section{Savannah River Site 2006}

\section{Absence Data}

Appendix K. Age-Adjusted IIIness and Injury Rates by Diagnostic Category*

Part 1. Men

\begin{tabular}{|c|c|c|c|c|c|}
\hline & & $\begin{array}{l}\text { Number of } \\
\text { Diagnoses }\end{array}$ & $\begin{array}{c}\text { Age-Adjusted } \\
\text { Rate per } 1,000 * *\end{array}$ & $\begin{array}{c}\text { Lower } 95 \% \\
\text { Confidence } \\
\text { Limit per 1,000 }\end{array}$ & $\begin{array}{c}\text { Upper 95\% } \\
\text { Confidence } \\
\text { Limit per 1,000 }\end{array}$ \\
\hline Diagnostic Category & ICD-9-CM Code & & & & \\
\hline $\begin{array}{l}\text { SYMPTOMS, SIGNS, \& ILL-DEFINED } \\
\text { CONDITIONS }\end{array}$ & 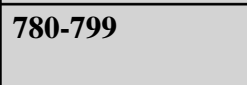 & 464 & 52.8 & 45.2 & 61.6 \\
\hline -Symptoms & $780-789$ & 445 & 51.6 & 44.0 & 60.4 \\
\hline -Non-Specific Abnormal Findings & $790-796$ & 17 & 1.1 & 0.7 & 1.8 \\
\hline -Ill-Defined \& Unknown Causes & \begin{tabular}{|l|}
$797-799$ \\
\end{tabular} & 2 & 0.1 & 0.0 & 0.5 \\
\hline INJURY \& POISONING & \begin{tabular}{|l|}
$800-999$ \\
\end{tabular} & 276 & 36.5 & 28.9 & 46.0 \\
\hline -Fracture - Skull & $800-804$ & 14 & 0.9 & 0.6 & 1.6 \\
\hline -Fracture - Upper Limb & 810-819 & 9 & 1.9 & 0.5 & 7.7 \\
\hline -Fracture - Lower Limb & $820-829$ & 11 & 1.0 & 0.5 & 1.9 \\
\hline -Dislocation & \begin{tabular}{|l|}
$830-839$ \\
\end{tabular} & 51 & 8.5 & 4.8 & 15.0 \\
\hline -Sprains \& Strains - Back & \begin{tabular}{|l|}
$846-847$ \\
\end{tabular} & 23 & 3.6 & 2.1 & 6.2 \\
\hline -Sprains \& Strains - Other & $840-845,848$ & 51 & 4.6 & 3.3 & 6.6 \\
\hline -Intracranial Injury & $850-854$ & 2 & 0.1 & 0.0 & 0.6 \\
\hline -Internal Injury - Thorax, Abdomen, Pelvis & \begin{tabular}{|c|}
$860-869$ \\
\end{tabular} & 2 & 0.2 & 0.0 & 0.7 \\
\hline -Open Wound - Head, Neck, Trunk & \begin{tabular}{|c|}
$870-879$ \\
\end{tabular} & 25 & 1.9 & 1.2 & 3.2 \\
\hline -Late Effects of Accident & $905-909$ & 1 & 0.1 & 0.0 & 0.6 \\
\hline -Superficial Injury & $910-919$ & 9 & 1.9 & 0.5 & 7.7 \\
\hline -Contusion & $920-924$ & 16 & 2.0 & 1.0 & 4.0 \\
\hline -Foreign Body Entering Orifice & $930-939$ & 2 & 0.3 & 0.1 & 1.2 \\
\hline -Burns & $940-949$ & 5 & 1.6 & 0.3 & 8.2 \\
\hline -Complications \& Unspecified Injuries & 958-959 & 1 & 0.1 & 0.0 & 0.6 \\
\hline -Toxic Effects - Non-medicinal & \begin{tabular}{|c|}
$980-989$ \\
\end{tabular} & 4 & 2.2 & 0.6 & 8.0 \\
\hline -Unspecified Effects - External Causes & $990-995$ & 16 & 3.1 & 1.2 & 7.8 \\
\hline -Complications of Surgical/Medical Care & \begin{tabular}{|c|}
$996-999$ \\
\end{tabular} & 34 & 2.5 & 1.7 & 3.5 \\
\hline Total & & 2866 & 333.4 & 313.3 & 354.8 \\
\hline
\end{tabular}

* Only those diagnostic categories with at least one occurrence appear in this table.

**Standardized to age distribution of 2000 U.S. population. 


\section{Savannah River Site 2006}

\section{Absence Data}

Appendix K. Age-Adjusted IIIness and Injury Rates by Diagnostic Category*

Part 2. Women

\begin{tabular}{|c|c|c|c|c|c|}
\hline & & $\begin{array}{c}\text { Number of } \\
\text { Diagnoses }\end{array}$ & $\begin{array}{c}\text { Age-Adjusted } \\
\text { Rate per 1,000** }\end{array}$ & $\begin{array}{c}\text { Lower } 95 \% \\
\text { Confidence } \\
\text { Limit per 1,000 }\end{array}$ & $\begin{array}{c}\text { Upper 95\% } \\
\text { Confidence } \\
\text { Limit per 1,000 }\end{array}$ \\
\hline Diagnostic Category & ICD-9-CM Code & & & & \\
\hline INFECTIOUS \& PARASITIC DISEASES (DIS) & 001-139 & 60 & 25.4 & 15.1 & 42.9 \\
\hline -Intestinal Infectious Dis & 001-009 & 25 & 7.7 & 4.5 & 13.1 \\
\hline -Other Bacterial Dis & 030-041 & 20 & 9.3 & 3.6 & 24.3 \\
\hline -Viral Dis with Exanthem & 050-057 & 2 & 4.5 & 0.7 & 29.5 \\
\hline -Other Viral Dis \& Chlamydiae & 070-079 & 7 & 1.6 & 0.8 & 3.3 \\
\hline -Mycoses & $110-118$ & 1 & 1.3 & 0.2 & 9.1 \\
\hline -Other Infections \& Parasitic Dis & $130-136$ & 5 & 1.0 & 0.4 & 2.5 \\
\hline MALIGNANT NEOPLASMS & $140-208,230-234$ & 17 & 5.8 & 3.0 & 11.4 \\
\hline -Lip, Oral Cavity, Pharynx & 140-149 & 2 & 0.4 & 0.1 & 1.7 \\
\hline -Digestive \& Peritoneal & 150-159 & 2 & 0.4 & 0.1 & 1.7 \\
\hline -Bone, Connective Tissue, Skin & 170-173, 176 & 2 & 0.4 & 0.1 & 1.8 \\
\hline -Breast & 174-175 & 4 & 3.0 & 0.9 & 10.0 \\
\hline -Other \& Unspecified Sites & $190,193-199$ & 7 & 1.6 & 0.7 & 3.3 \\
\hline BENIGN \& UNCERTAIN NEOPLASMS & 210-229, 235-239 & 45 & 13.6 & 9.3 & 19.8 \\
\hline ENDOCRINE/METABOLIC/IMMUNITY & 240-279 & 42 & 11.2 & 7.7 & 16.4 \\
\hline -Thyroid Gland Disorders & $240-246$ & 13 & 2.9 & 1.7 & 5.0 \\
\hline -Other Endocrine Gland Dis & $250-259$ & 10 & 4.1 & 1.7 & 9.6 \\
\hline -Other Metabolic \& Immunity Disorders & 270-279 & 19 & 4.2 & 2.7 & 6.7 \\
\hline BLOOD \& BLOOD-FORMING ORGANS & $280-289$ & 12 & 4.5 & 2.1 & 9.4 \\
\hline MENTAL DISORDERS & $290-319$ & 26 & 10.1 & 6.1 & 16.9 \\
\hline -Psychoses & 290-299 & 5 & 2.0 & 0.6 & 6.3 \\
\hline -Non-Psychotic Disorders & $300-302,306-316$ & 21 & 8.2 & 4.6 & 14.4 \\
\hline NERVOUS SYSTEM (NS) \& SENSE ORGANS & $320-389$ & 99 & 39.9 & 28.9 & 54.9 \\
\hline -Other Disorders of Central NS & $340-349$ & 24 & 11.7 & 6.9 & 19.6 \\
\hline -Disorders of Peripheral NS & $350-359$ & 28 & 10.5 & 6.4 & 17.2 \\
\hline -Disorders of Eye & $360-379$ & 27 & 12.1 & 5.6 & 26.4 \\
\hline -Dis of Ear \& Mastoid & 380-389 & 19 & 5.4 & 3.0 & 9.6 \\
\hline CIRCULATORY SYSTEM & $390-459$ & 59 & 21.9 & 15.6 & 30.9 \\
\hline -Hypertensive Dis & $401-405$ & 26 & 6.8 & 4.3 & 10.7 \\
\hline -Ischemic Heart Dis & $410-414$ & 5 & 1.1 & 0.5 & 2.6 \\
\hline -Dis of Pulmonary Circulation & $415-417$ & 1 & 0.2 & 0.0 & 1.5 \\
\hline -Other Heart Dis & $420-429$ & 9 & 1.9 & 1.0 & 3.7 \\
\hline -Cerebrovascular Dis & $430-438$ & 1 & 1.3 & 0.2 & 9.1 \\
\hline -Dis of Arteries \& Capillaries & $440-448$ & 2 & 0.5 & 0.1 & 1.9 \\
\hline -Dis of Veins, Lymphatics, Other & $451-459$ & 15 & 10.2 & 5.6 & 18.6 \\
\hline
\end{tabular}

(Continued)

*Only those diagnostic categories with at least one occurrence appear in this table.

**Standardized to age distribution of 2000 U.S. population. 


\section{Savannah River Site 2006}

\section{Absence Data}

Appendix K. Age-Adjusted IIIness and Injury Rates by Diagnostic Category*

Part 2. Women

\begin{tabular}{|c|c|c|c|c|c|}
\hline & & $\begin{array}{l}\text { Number of } \\
\text { Diagnoses }\end{array}$ & $\begin{array}{l}\text { Age-Adjusted } \\
\text { Rate per } 1,000 * *\end{array}$ & $\begin{array}{c}\text { Lower } 95 \% \\
\text { Confidence } \\
\text { Limit per 1,000 }\end{array}$ & $\begin{array}{c}\text { Upper } 95 \% \\
\text { Confidence } \\
\text { Limit per 1,000 }\end{array}$ \\
\hline Diagnostic Category & ICD-9-CM Code & \multirow[b]{2}{*}{388} & \multirow[b]{2}{*}{147.9} & \multirow[b]{2}{*}{123.7} & \multirow[b]{2}{*}{177.0} \\
\hline RESPIRATORY SYSTEM & $460-519$ & & & & \\
\hline -Acute Respiratory Infections & $460-466$ & 258 & 91.9 & 74.2 & 113.8 \\
\hline -Other Dis Upper Respiratory Tract & $470-478$ & 69 & 30.2 & 19.1 & 47.8 \\
\hline -Pneumonia \& Influenza & $480-487$ & 42 & 19.7 & 11.5 & 33.7 \\
\hline -Chronic Obstructive Dis & $490-496$ & 17 & 5.7 & 3.0 & 10.9 \\
\hline -Other Respiratory Dis & $510-519$ & 2 & 0.4 & 0.1 & 1.7 \\
\hline DIGESTIVE SYSTEM & $520-579$ & 106 & 43.3 & 33.5 & 56.0 \\
\hline -Oral Cavity, Saliva Glands, Jaw & $520-529$ & 24 & 10.0 & 5.9 & 17.0 \\
\hline -Esophagus, Stomach, Duodenum & $530-537$ & 25 & 7.1 & 4.2 & 11.8 \\
\hline -Appendicitis & $540-543$ & 5 & 1.1 & 0.5 & 2.6 \\
\hline -Hernia & $550-553$ & 4 & 0.8 & 0.3 & 2.2 \\
\hline -Enteritis, Colitis & $555-558$ & 14 & 8.7 & 4.5 & 16.7 \\
\hline -Other Intestinal Dis & $560-569$ & 25 & 13.6 & 8.2 & 22.6 \\
\hline -Other Digestive Dis & $570-579$ & 9 & 2.0 & 1.0 & 3.8 \\
\hline GENITOURINARY SYSTEM & $580-629$ & 137 & 59.2 & 46.0 & 76.2 \\
\hline -Other Urinary Dis & $590-599$ & 41 & 21.5 & 14.6 & 31.8 \\
\hline -Breast Disorders & $610-611$ & 9 & 6.1 & 1.5 & 24.8 \\
\hline -Pelvic Inflammatory Dis & 614-616 & 4 & 1.8 & 0.5 & 6.4 \\
\hline -Other Female Disorders & $617-629$ & 83 & 29.8 & 22.3 & 39.8 \\
\hline PREGNANCY \& CHILDBIRTH & $630-677$ & 8 & 8.7 & 4.4 & 17.4 \\
\hline -Pregnancy with Abortive Outcome & 634-639 & 8 & 8.7 & 4.4 & 17.4 \\
\hline SKIN \& SUBCUTANEOUS TISSUE & $680-709$ & 17 & 6.3 & 3.4 & 12.0 \\
\hline -Infections & $680-686$ & 3 & 0.7 & 0.2 & 2.0 \\
\hline -Other & 700-709 & 14 & 5.7 & 2.8 & 11.4 \\
\hline $\begin{array}{l}\text { MUSCULOSKELETAL \& CONNECTIVE } \\
\text { TISSUE }\end{array}$ & $710-739$ & 289 & 115.7 & 94.7 & 141.3 \\
\hline -Arthropathies & 710-719 & 57 & 24.1 & 15.2 & 38.3 \\
\hline -Dorsopathies & $720-724$ & 156 & 74.3 & 56.9 & 97.0 \\
\hline -Rheumatism, Excluding Back & $725-729$ & 63 & 14.4 & 10.9 & 18.9 \\
\hline -Other Dis \& Acquired Deformities & 730-739 & 13 & 2.8 & 1.6 & 4.9 \\
\hline CONGENITAL ANOMALIES & $740-759$ & 1 & 0.2 & 0.0 & 1.5 \\
\hline $\begin{array}{l}\text { SYMPTOMS, SIGNS, \& ILL-DEFINED } \\
\text { CONDITIONS }\end{array}$ & 780-799 & 303 & 120.7 & 100.5 & 144.9 \\
\hline -Symptoms & $780-789$ & 295 & 117.0 & 97.1 & 140.9 \\
\hline -Non-Specific Abnormal Findings & $790-796$ & 8 & 3.7 & 1.4 & 9.4 \\
\hline INJURY \& POISONING & $800-999$ & 100 & 47.2 & 32.7 & 68.1 \\
\hline
\end{tabular}

(Continued)

*Only those diagnostic categories with at least one occurrence appear in this table.

**Standardized to age distribution of 2000 U.S. population. 


\section{Savannah River Site 2006}

\section{Absence Data}

Appendix K. Age-Adjusted Illness and Injury Rates by Diagnostic Category*

Part 2. Women

\begin{tabular}{|c|c|c|c|c|c|}
\hline & & $\begin{array}{l}\text { Number of } \\
\text { Diagnoses }\end{array}$ & $\begin{array}{c}\text { Age-Adjusted } \\
\text { Rate per } 1,000 * *\end{array}$ & $\begin{array}{c}\text { Lower } 95 \% \\
\text { Confidence } \\
\text { Limit per 1,000 }\end{array}$ & $\begin{array}{c}\text { Upper } 95 \% \\
\text { Confidence } \\
\text { Limit per 1,000 }\end{array}$ \\
\hline Diagnostic Category & ICD-9-CM Code & \multirow[b]{2}{*}{3} & \multirow[b]{2}{*}{0.7} & \multirow[b]{2}{*}{0.2} & \multirow[b]{2}{*}{2.2} \\
\hline -Fracture - Skull & $800-804$ & & & & \\
\hline -Fracture - Upper Limb & 810-819 & 2 & 1.3 & 0.3 & 6.9 \\
\hline -Fracture - Lower Limb & 820-829 & 5 & 1.1 & 0.5 & 2.6 \\
\hline -Dislocation & $830-839$ & 11 & 3.3 & 1.5 & 7.1 \\
\hline -Sprains \& Strains - Back & 846-847 & 12 & 12.6 & 4.7 & 33.8 \\
\hline -Sprains \& Strains - Other & $840-845,848$ & 15 & 4.2 & 2.2 & 8.0 \\
\hline -Intracranial Injury & $850-854$ & 1 & 0.2 & 0.0 & 1.7 \\
\hline -Open Wound - Head, Neck, Trunk & $870-879$ & 4 & 0.9 & 0.3 & 2.4 \\
\hline -Late Effects of Accident & $905-909$ & 1 & 0.2 & 0.0 & 1.7 \\
\hline -Superficial Injury & $910-919$ & 1 & 0.2 & 0.0 & 1.7 \\
\hline -Contusion & $920-924$ & 1 & 4.3 & 0.6 & 30.7 \\
\hline -Crushing Injury & $925-929$ & 1 & 0.2 & 0.0 & 1.7 \\
\hline -Burns & $940-949$ & 6 & 1.4 & 0.6 & 3.1 \\
\hline -Unspecified Effects - External Causes & $990-995$ & 8 & 1.8 & 0.9 & 3.6 \\
\hline -Complications of Surgical/Medical Care & 996-999 & 29 & 14.5 & 9.0 & 23.4 \\
\hline Total & & 1709 & 681.7 & 629.8 & 737.9 \\
\hline
\end{tabular}

*Only those diagnostic categories with at least one occurrence appear in this table.

**Standardized to age distribution of 2000 U.S. population. 


\section{Savannah River Site 2006}

\section{Absence Data}

Appendix K. Age-Adjusted IIIness and Injury Rates by Diagnostic Category*

Part 3. Men and Women

\begin{tabular}{|c|c|c|c|c|c|}
\hline & & $\begin{array}{l}\text { Number of } \\
\text { Diagnoses }\end{array}$ & $\begin{array}{c}\text { Age-Adjusted } \\
\text { Rate per } 1,000 * *\end{array}$ & $\begin{array}{c}\text { Lower } 95 \% \\
\text { Confidence } \\
\text { Limit per 1,000 }\end{array}$ & $\begin{array}{c}\text { Upper } 95 \% \\
\text { Confidence } \\
\text { Limit per 1,000 }\end{array}$ \\
\hline Diagnostic Category & ICD-9-CM Code & & & & \\
\hline INFECTIOUS \& PARASITIC DISEASES (DIS) & 001-139 & 146 & 14.5 & 10.6 & 19.9 \\
\hline -Intestinal Infectious Dis & 001-009 & 55 & 3.8 & 2.7 & 5.3 \\
\hline -Other Bacterial Dis & 030-041 & 49 & 5.9 & 3.4 & 10.1 \\
\hline -Viral Dis with Exanthem & 050-057 & 9 & 2.4 & 0.8 & 7.7 \\
\hline -Other Viral Dis \& Chlamydiae & 070-079 & 22 & 1.4 & 0.8 & 2.4 \\
\hline -Syphilis \& Other Venereal Dis & 090-099 & 1 & 0.0 & 0.0 & 0.3 \\
\hline -Mycoses & 110-118 & 5 & 0.7 & 0.3 & 2.0 \\
\hline -Other Infections \& Parasitic Dis & $130-136$ & 5 & 0.2 & 0.1 & 0.5 \\
\hline MALIGNANT NEOPLASMS & $140-208,230-234$ & 76 & 5.3 & 4.0 & 6.9 \\
\hline -Lip, Oral Cavity, Pharynx & $140-149$ & 13 & 0.6 & 0.3 & 1.0 \\
\hline -Digestive \& Peritoneal & $150-159$ & 6 & 0.5 & 0.2 & 1.4 \\
\hline -Respiratory \& Intrathoracic & $160-165$ & 3 & 0.4 & 0.1 & 1.4 \\
\hline -Bone, Connective Tissue, Skin & $170-173,176$ & 17 & 1.0 & 0.6 & 1.7 \\
\hline -Breast & 174-175 & 4 & 0.4 & 0.1 & 1.4 \\
\hline -Genitourinary & 179-189 & 10 & 0.9 & 0.4 & 1.8 \\
\hline -Other \& Unspecified Sites & 190, 193-199 & 22 & 1.4 & 0.8 & 2.2 \\
\hline -Lymphatic \& Hematopoietic & $200-208$ & 1 & 0.0 & 0.0 & 0.3 \\
\hline BENIGN \& UNCERTAIN NEOPLASMS & $210-229,235-239$ & 67 & 5.1 & 3.7 & 7.0 \\
\hline ENDOCRINE/METABOLIC/IMMUNITY & 240-279 & 101 & 8.3 & 5.6 & 12.1 \\
\hline -Thyroid Gland Disorders & $240-246$ & 18 & 1.0 & 0.6 & 1.5 \\
\hline -Other Endocrine Gland Dis & 250-259 & 30 & 4.0 & 1.9 & 8.4 \\
\hline -Other Metabolic \& Immunity Disorders & 270-279 & 53 & 3.3 & 2.4 & 4.5 \\
\hline BLOOD \& BLOOD-FORMING ORGANS & 280-289 & 29 & 2.0 & 1.3 & 3.3 \\
\hline MENTAL DISORDERS & 290-319 & 73 & 8.3 & 5.5 & 12.5 \\
\hline -Psychoses & 290-299 & 12 & 1.2 & 0.6 & 2.6 \\
\hline -Non-Psychotic Disorders & $300-302,306-316$ & 58 & 6.8 & 4.2 & 11.0 \\
\hline -Alcohol Dependence & 303 & 1 & 0.1 & 0.0 & 0.4 \\
\hline -Drug Dependence & 304-305 & 2 & 0.2 & 0.1 & 1.1 \\
\hline NERVOUS SYSTEM (NS) \& SENSE ORGANS & 320-389 & 254 & 21.2 & 17.8 & 25.4 \\
\hline -Inflammatory Dis of Central NS & $320-326$ & 2 & 0.1 & 0.0 & 0.4 \\
\hline -Hereditary/Degenerative Central NS Dis & 330-337 & 5 & 0.2 & 0.1 & 0.5 \\
\hline -Other Disorders of Central NS & 340-349 & 39 & 3.9 & 2.6 & 5.9 \\
\hline -Disorders of Peripheral NS & 350-359 & 58 & 5.1 & 3.6 & 7.2 \\
\hline -Disorders of Eye & $360-379$ & 90 & 8.1 & 5.8 & 11.2 \\
\hline -Dis of Ear \& Mastoid & 380-389 & 58 & 3.8 & 2.8 & 5.2 \\
\hline
\end{tabular}

(Continued)

*Only those diagnostic categories with at least one occurrence appear in this table.

**Standardized to age distribution of 2000 U.S. population. 


\section{Savannah River Site 2006}

\section{Absence Data}

Appendix K. Age-Adjusted IIIness and Injury Rates by Diagnostic Category*

Part 3. Men and Women

\begin{tabular}{|c|c|c|c|c|c|}
\hline & & $\begin{array}{l}\text { Number of } \\
\text { Diagnoses }\end{array}$ & $\begin{array}{c}\text { Age-Adjusted } \\
\text { Rate per } 1,000 * *\end{array}$ & $\begin{array}{c}\text { Lower } 95 \% \\
\text { Confidence } \\
\text { Limit per 1,000 }\end{array}$ & $\begin{array}{c}\text { Upper } 95 \% \\
\text { Confidence } \\
\text { Limit per 1,000 }\end{array}$ \\
\hline Diagnostic Category & ICD-9-CM Code & \multirow[b]{2}{*}{261} & \multirow[b]{2}{*}{21.0} & \multirow[b]{2}{*}{17.9} & \multirow[b]{2}{*}{24.7} \\
\hline CIRCULATORY SYSTEM & $390-459$ & & & & \\
\hline -Hypertensive Dis & $401-405$ & 75 & 6.8 & 5.1 & 9.2 \\
\hline -Ischemic Heart Dis & $410-414$ & 60 & 4.2 & 3.0 & 5.9 \\
\hline -Dis of Pulmonary Circulation & $415-417$ & 4 & 0.2 & 0.1 & 0.5 \\
\hline -Other Heart Dis & $420-429$ & 37 & 2.3 & 1.6 & 3.3 \\
\hline -Cerebrovascular Dis & $430-438$ & 19 & 1.4 & 0.8 & 2.3 \\
\hline -Dis of Arteries \& Capillaries & $440-448$ & 12 & 0.7 & 0.4 & 1.4 \\
\hline -Dis of Veins, Lymphatics, Other & $451-459$ & 54 & 5.5 & 3.8 & 7.9 \\
\hline RESPIRATORY SYSTEM & $460-519$ & 998 & 94.3 & 85.0 & 104.6 \\
\hline -Acute Respiratory Infections & $460-466$ & 611 & 54.7 & 48.1 & 62.3 \\
\hline -Other Dis Upper Respiratory Tract & $470-478$ & 211 & 21.8 & 17.5 & 27.2 \\
\hline -Pneumonia \& Influenza & $480-487$ & 147 & 15.9 & 11.9 & 21.4 \\
\hline -Chronic Obstructive Dis & $490-496$ & 26 & 1.7 & 1.0 & 2.8 \\
\hline -Lung Dis from External Agents & $500-508$ & 1 & 0.0 & 0.0 & 0.3 \\
\hline -Other Respiratory Dis & $510-519$ & 2 & 0.1 & 0.0 & 0.4 \\
\hline DIGESTIVE SYSTEM & $520-579$ & 329 & 25.9 & 22.1 & 30.4 \\
\hline -Oral Cavity, Saliva Glands, Jaw & $520-529$ & 69 & 5.4 & 3.9 & 7.4 \\
\hline -Esophagus, Stomach, Duodenum & $\mathbf{5 3 0 - 5 3 7}$ & 66 & 4.0 & 3.0 & 5.5 \\
\hline -Appendicitis & $540-543$ & 10 & 0.7 & 0.3 & 1.7 \\
\hline -Hernia & $550-553$ & 58 & 4.6 & 2.8 & 7.6 \\
\hline -Enteritis, Colitis & $555-558$ & 30 & 3.0 & 1.9 & 4.8 \\
\hline -Other Intestinal Dis & $560-569$ & 59 & 5.4 & 3.9 & 7.6 \\
\hline -Other Digestive Dis & $570-579$ & 37 & 2.6 & 1.7 & 4.0 \\
\hline GENITOURINARY SYSTEM & $580-629$ & 230 & 25.4 & 20.5 & 31.4 \\
\hline -Other Urinary Dis & $590-599$ & 109 & 14.2 & 10.4 & 19.4 \\
\hline -Male Genital Organ Dis & $600-608$ & 24 & 2.0 & 1.2 & 3.3 \\
\hline -Breast Disorders & 610-611 & 10 & 1.5 & 0.4 & 5.7 \\
\hline -Pelvic Inflammatory Dis & 614-616 & 4 & 0.5 & 0.1 & 1.7 \\
\hline -Other Female Disorders & $617-629$ & 83 & 7.1 & 5.4 & 9.4 \\
\hline PREGNANCY \& CHILDBIRTH & $630-677$ & 8 & 2.3 & 1.1 & 4.5 \\
\hline -Pregnancy with Abortive Outcome & 634-639 & 8 & 2.3 & 1.1 & 4.5 \\
\hline SKIN \& SUBCUTANEOUS TISSUE & 680-709 & 52 & 4.7 & 3.3 & 6.8 \\
\hline -Infections & $680-686$ & 22 & 1.5 & 0.9 & 2.4 \\
\hline -Other Inflammatory Conditions & 690-698 & 1 & 0.1 & 0.0 & 0.4 \\
\hline -Other & 700-709 & 29 & 3.2 & 2.0 & 5.1 \\
\hline
\end{tabular}

(Continued)

*Only those diagnostic categories with at least one occurrence appear in this table.

**Standardized to age distribution of 2000 U.S. population. 


\section{Savannah River Site 2006}

\section{Absence Data}

Appendix K. Age-Adjusted IIIness and Injury Rates by Diagnostic Category*

Part 3. Men and Women

\begin{tabular}{|c|c|c|c|c|c|}
\hline & & $\begin{array}{l}\text { Number of } \\
\text { Diagnoses }\end{array}$ & $\begin{array}{l}\text { Age-Adjusted } \\
\text { Rate per } 1,000 * *\end{array}$ & $\begin{array}{c}\text { Lower } 95 \% \\
\text { Confidence } \\
\text { Limit per 1,000 }\end{array}$ & $\begin{array}{c}\text { Upper } 95 \% \\
\text { Confidence } \\
\text { Limit per 1,000 }\end{array}$ \\
\hline Diagnostic Category & ICD-9-CM Code & & & & \\
\hline $\begin{array}{l}\text { MUSCULOSKELETAL \& CONNECTIVE } \\
\text { TISSUE }\end{array}$ & $710-739$ & 804 & 69.9 & 62.5 & 78.3 \\
\hline -Arthropathies & $710-719$ & 176 & 18.0 & 14.1 & 23.1 \\
\hline -Dorsopathies & $720-724$ & 450 & 40.2 & 34.7 & 46.5 \\
\hline -Rheumatism, Excluding Back & $725-729$ & 153 & 10.2 & 7.9 & 13.2 \\
\hline -Other Dis \& Acquired Deformities & 730-739 & 25 & 1.5 & 0.9 & 2.5 \\
\hline CONGENITAL ANOMALIES & 740-759 & 4 & 0.2 & 0.1 & 0.6 \\
\hline $\begin{array}{l}\text { SYMPTOMS, SIGNS, \& ILL-DEFINED } \\
\text { CONDITIONS }\end{array}$ & 780-799 & 767 & 68.8 & 61.2 & 77.3 \\
\hline -Symptoms & 780-789 & 740 & 67.0 & 59.5 & 75.5 \\
\hline -Non-Specific Abnormal Findings & $790-796$ & 25 & 1.6 & 1.0 & 2.7 \\
\hline -IIl-Defined \& Unknown Causes & 797-799 & 2 & 0.1 & 0.0 & 0.4 \\
\hline INJURY \& POISONING & $800-999$ & 376 & 39.3 & 32.4 & 47.6 \\
\hline -Fracture - Skull & 800-804 & 17 & 0.9 & 0.6 & 1.5 \\
\hline -Fracture - Upper Limb & 810-819 & 11 & 1.8 & 0.6 & 5.7 \\
\hline -Fracture - Lower Limb & $820-829$ & 16 & 1.1 & 0.6 & 1.9 \\
\hline -Dislocation & 830-839 & 62 & 7.3 & 4.4 & 12.1 \\
\hline -Sprains \& Strains - Back & 846-847 & 35 & 5.7 & 3.2 & 10.1 \\
\hline -Sprains \& Strains - Other & $840-845,848$ & 66 & 4.7 & 3.5 & 6.4 \\
\hline -Intracranial Injury & $850-854$ & 3 & 0.2 & 0.1 & 0.5 \\
\hline -Internal Injury - Thorax, Abdomen, Pelvis & $860-869$ & 2 & 0.1 & 0.0 & 0.5 \\
\hline -Open Wound - Head, Neck, Trunk & 870-879 & 29 & 1.7 & 1.1 & 2.7 \\
\hline -Late Effects of Accident & 905-909 & 2 & 0.1 & 0.0 & 0.5 \\
\hline -Superficial Injury & 910-919 & 10 & 1.5 & 0.4 & 5.7 \\
\hline -Contusion & $920-924$ & 17 & 2.5 & 1.0 & 6.1 \\
\hline -Crushing Injury & 925-929 & 1 & 0.1 & 0.0 & 0.4 \\
\hline -Foreign Body Entering Orifice & 930-939 & 2 & 0.2 & 0.0 & 1.1 \\
\hline -Burns & $940-949$ & 11 & 1.6 & 0.5 & 5.6 \\
\hline -Complications \& Unspecified Injuries & 958-959 & 1 & 0.1 & 0.0 & 0.4 \\
\hline -Toxic Effects - Non-medicinal & $980-989$ & 4 & 1.6 & 0.4 & 6.0 \\
\hline -Unspecified Effects - External Causes & $990-995$ & 24 & 2.8 & 1.3 & 6.1 \\
\hline -Complications of Surgical/Medical Care & 996-999 & 63 & 5.3 & 3.8 & 7.3 \\
\hline Total & & 4575 & 416.4 & 396.8 & 437.1 \\
\hline
\end{tabular}

* Only those diagnostic categories with at least one occurrence appear in this table.

**Standardized to age distribution of 2000 U.S. population. 
Savannah River Site 2006

OSHA Data

Appendix L. Number of Workers with at Least One OSHA Event by Gender, Age, and Job Category*

\begin{tabular}{|c|c|c|c|c|c|c|c|c|c|}
\hline \multirow{3}{*}{ Job Category } & \multicolumn{3}{|c|}{ Women } & \multicolumn{5}{|c|}{ Men } & \multirow[b]{3}{*}{ TOTAL } \\
\hline & \multicolumn{2}{|c|}{ Age Group } & \multirow[b]{2}{*}{ TOTAL } & \multicolumn{4}{|c|}{ Age Group } & \multirow[b]{2}{*}{ TOTAL } & \\
\hline & $40-49$ & $50+$ & & $16-29$ & 30 - 39 & $40-49$ & $50+$ & & \\
\hline Professional & 1 & 2 & 3 & 1 & 2 & 3 & 3 & 9 & 12 \\
\hline Technical Support & 2 & 1 & 3 & 0 & 2 & 4 & 4 & 10 & 13 \\
\hline Service & 0 & 0 & 0 & 0 & 0 & 3 & 4 & 7 & 7 \\
\hline Security and Fire & 0 & 0 & 0 & 0 & 0 & 1 & 1 & 2 & 2 \\
\hline Crafts & 0 & 0 & 0 & 1 & 1 & 0 & 3 & 5 & 5 \\
\hline Line Operators & 2 & 2 & 4 & 0 & 0 & 3 & 3 & 6 & 10 \\
\hline TOTAL & 5 & 5 & 10 & 2 & 5 & 14 & 18 & 39 & 49 \\
\hline
\end{tabular}

*Only those job categories and gender/age combinations with at least one OSHA event appear in this table.

Appendix M. Total Number of Workdays Lost or with Restricted Activity from OSHA Events by Gender and Age

\begin{tabular}{|c|c|c|c|c|c|c|c|c|c|}
\hline \multirow[b]{2}{*}{ Age Group } & \multicolumn{3}{|c|}{ Women } & \multicolumn{3}{|c|}{ Men } & \multicolumn{3}{|c|}{ TOTAL } \\
\hline & $\begin{array}{c}\text { Number of } \\
\text { Events }\end{array}$ & $\begin{array}{c}\text { Days } \\
\text { Restricted }\end{array}$ & Days Lost & $\begin{array}{c}\text { Number of } \\
\text { Events }\end{array}$ & \begin{tabular}{|c|} 
Days \\
Restricted
\end{tabular} & Days Lost & $\begin{array}{c}\text { Number of } \\
\text { Events }\end{array}$ & $\begin{array}{c}\text { Days } \\
\text { Restricted }\end{array}$ & Days Lost \\
\hline $16-29$ & 0 & 0 & 0 & 2 & 41 & 0 & 2 & 41 & 0 \\
\hline $30-39$ & 0 & 0 & 0 & 6 & 0 & 0 & 6 & 0 & 0 \\
\hline $40-49$ & 5 & 2 & 0 & 14 & 121 & 0 & 19 & 123 & 0 \\
\hline $50+$ & 5 & 33 & 0 & 18 & 204 & 46 & 23 & 237 & 46 \\
\hline TOTAL & 10 & 35 & 0 & 40 & 366 & 46 & 50 & 401 & 46 \\
\hline
\end{tabular}

Appendix N. Total Number of Workdays Lost or with Restricted Activity from OSHA Events by Gender and Job Category*

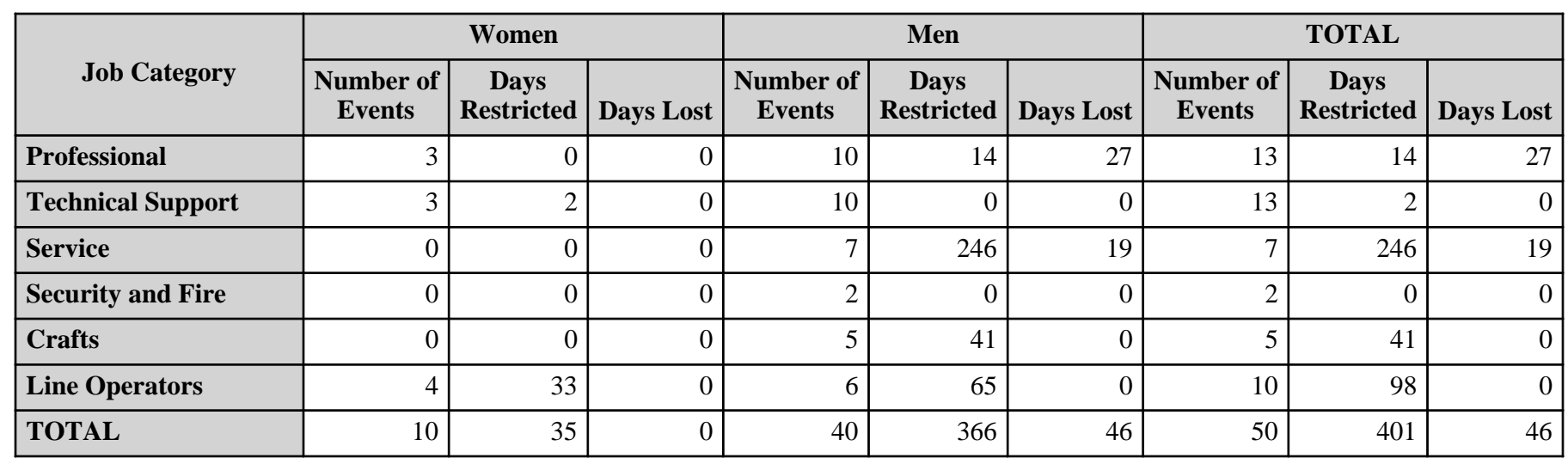

*Only those job categories with at least one OSHA event appear in this table. 


\section{Savannah River Site 2006}

\section{OSHA Data}

Appendix O. Number of Diagnoses in Each Diagnostic Category by Gender and Age*

\begin{tabular}{|c|c|c|c|c|}
\hline & & \multicolumn{3}{|c|}{ Women } \\
\hline & & \multicolumn{2}{|c|}{ Age Group } & \multirow[b]{2}{*}{ TOTAI } \\
\hline & & $40-49$ & $50+$ & \\
\hline Diagnostic Category & ICD-9-CM code & \multirow[b]{2}{*}{0} & \multirow[b]{2}{*}{0} & \multirow[b]{2}{*}{0} \\
\hline NERVOUS SYSTEM (NS) \& SENSE ORGANS & $320-389$ & & & \\
\hline -Disorders of Eye & $360-379$ & 0 & 0 & 0 \\
\hline RESPIRATORY SYSTEM & $460-519$ & 1 & 2 & 3 \\
\hline -Chronic Obstructive Dis & $490-496$ & 0 & 1 & 1 \\
\hline -Lung Dis from External Agents & \begin{tabular}{|c|}
$500-508$ \\
\end{tabular} & 1 & 1 & 2 \\
\hline DIGESTIVE SYSTEM & $520-579$ & 0 & 1 & 1 \\
\hline -Oral Cavity, Saliva Glands, Jaw & $520-529$ & 0 & 1 & 1 \\
\hline -Hernia & \begin{tabular}{|c|}
$550-553$ \\
\end{tabular} & 0 & 0 & 0 \\
\hline $\begin{array}{l}\text { MUSCULOSKELETAL \& CONNECTIVE } \\
\text { TISSUE }\end{array}$ & $710-739$ & 0 & 0 & 0 \\
\hline -Arthropathies & $710-719$ & 0 & 0 & 0 \\
\hline -Dorsopathies & $720-724$ & 0 & 0 & 0 \\
\hline -Rheumatism, Excluding Back & 725-729 & 0 & 0 & 0 \\
\hline $\begin{array}{l}\text { SYMPTOMS, SIGNS, \& ILL-DEFINED } \\
\text { CONDITIONS }\end{array}$ & $780-799$ & 1 & 2 & 3 \\
\hline -Symptoms & $780-789$ & 1 & 2 & 3 \\
\hline INJURY \& POISONING & $800-999$ & 4 & 6 & 10 \\
\hline -Fracture - Neck, Trunk & $805-809$ & 1 & 0 & 1 \\
\hline -Fracture - Upper Limb & 810-819 & 0 & 0 & 0 \\
\hline -Sprains \& Strains - Back & 846-847 & 0 & 0 & 0 \\
\hline -Sprains \& Strains - Other & $840-845,848$ & 1 & 2 & 3 \\
\hline -Open Wound - Head, Neck, Trunk & $870-879$ & 0 & 1 & 1 \\
\hline -Open Wound - Upper Limb & 880-887 & 1 & 0 & 1 \\
\hline -Superficial Injury & $910-919$ & 0 & 1 & 1 \\
\hline -Contusion & $920-924$ & 0 & 2 & 2 \\
\hline -Foreign Body Entering Orifice & 930-939 & 0 & 0 & 0 \\
\hline -Burns & $940-949$ & 0 & 0 & 0 \\
\hline -Unspecified Effects - External Causes & $990-995$ & 1 & 0 & 1 \\
\hline
\end{tabular}

*Only those diagnostic categories and gender/age combinations with at least one OSHA event appear in this table. 


\section{Savannah River Site 2006}

\section{OSHA Data}

Appendix O. Number of Diagnoses in Each Diagnostic Category by Gender and Age*

\begin{tabular}{|c|c|c|c|c|c|c|c|}
\hline & & \multicolumn{5}{|c|}{ Men } & \multirow[b]{3}{*}{ TOTAL } \\
\hline & & \multicolumn{4}{|c|}{ Age Group } & \multirow[b]{2}{*}{ TOTAL } & \\
\hline & & $16-29$ & 30 - 39 & $40-49$ & $50+$ & & \\
\hline Diagnostic Category & ICD-9-CM code & & & & & & \\
\hline NERVOUS SYSTEM (NS) \& SENSE ORGANS & $320-389$ & 0 & 0 & 0 & 1 & 1 & 1 \\
\hline -Disorders of Eye & $360-379$ & 0 & 0 & 0 & 1 & 1 & 1 \\
\hline RESPIRATORY SYSTEM & $460-519$ & 0 & 0 & 1 & 4 & 5 & 8 \\
\hline -Chronic Obstructive Dis & $490-496$ & 0 & 0 & 0 & 1 & 1 & 2 \\
\hline -Lung Dis from External Agents & $500-508$ & 0 & 0 & 1 & 3 & 4 & 6 \\
\hline DIGESTIVE SYSTEM & $520-579$ & 1 & 0 & 0 & 1 & 2 & 3 \\
\hline -Oral Cavity, Saliva Glands, Jaw & $520-529$ & 0 & 0 & 0 & 0 & 0 & 1 \\
\hline -Hernia & $550-553$ & 1 & 0 & 0 & 1 & 2 & 2 \\
\hline $\begin{array}{l}\text { MUSCULOSKELETAL \& CONNECTIVE } \\
\text { TISSUE }\end{array}$ & $710-739$ & 0 & 1 & 2 & 3 & 6 & 6 \\
\hline -Arthropathies & $710-719$ & 0 & 1 & 1 & 1 & 3 & 3 \\
\hline -Dorsopathies & $720-724$ & 0 & 0 & 1 & 1 & 2 & 2 \\
\hline -Rheumatism, Excluding Back & $725-729$ & 0 & 0 & 0 & 1 & 1 & 1 \\
\hline $\begin{array}{l}\text { SYMPTOMS, SIGNS, \& ILL-DEFINED } \\
\text { CONDITIONS }\end{array}$ & $780-799$ & 0 & 0 & 1 & 3 & 4 & 7 \\
\hline -Symptoms & $780-789$ & 0 & 0 & 1 & 3 & 4 & 7 \\
\hline INJURY \& POISONING & 800-999 & 1 & 7 & 10 & 18 & 36 & 46 \\
\hline -Fracture - Neck, Trunk & \begin{tabular}{|c|}
$805-809$ \\
\end{tabular} & 0 & 0 & 0 & 0 & 0 & 1 \\
\hline -Fracture - Upper Limb & $810-819$ & 0 & 0 & 1 & 0 & 1 & 1 \\
\hline -Sprains \& Strains - Back & 846-847 & 0 & 1 & 1 & 0 & 2 & 2 \\
\hline -Sprains \& Strains - Other & $840-845,848$ & 1 & 0 & 1 & 1 & 3 & 6 \\
\hline -Open Wound - Head, Neck, Trunk & $870-879$ & 0 & 0 & 1 & 0 & 1 & 2 \\
\hline -Open Wound - Upper Limb & 880-887 & 0 & 2 & 4 & 3 & 9 & 10 \\
\hline -Superficial Injury & $910-919$ & 0 & 1 & 1 & 6 & 8 & 9 \\
\hline -Contusion & $920-924$ & 0 & 3 & 0 & 4 & 7 & 9 \\
\hline -Foreign Body Entering Orifice & 930-939 & 0 & 0 & 1 & 1 & 2 & 2 \\
\hline -Burns & $940-949$ & 0 & 0 & 0 & 3 & 3 & 3 \\
\hline -Unspecified Effects - External Causes & $990-995$ & 0 & 0 & 0 & 0 & 0 & 1 \\
\hline
\end{tabular}

\begin{tabular}{|c|c|c|c|c|c|c|c|c|c|}
\hline & \multicolumn{3}{|c|}{ Women } & \multicolumn{5}{|c|}{ Men } & \multirow[b]{3}{*}{ TOTAL } \\
\hline & \multicolumn{2}{|c|}{ Age Group } & \multirow[b]{2}{*}{ TOTAL } & \multicolumn{4}{|c|}{ Age Group } & \multirow[b]{2}{*}{ TOTAL } & \\
\hline & $40-49$ & $50+$ & & $16-29$ & $30-39$ & $40-49$ & $50+$ & & \\
\hline Diagnostic Category & \multirow[b]{2}{*}{6} & \multirow[b]{2}{*}{11} & \multirow[b]{2}{*}{17} & \multirow[b]{2}{*}{2} & \multirow[b]{2}{*}{8} & \multirow[b]{2}{*}{14} & \multirow[b]{2}{*}{30} & \multirow[b]{2}{*}{54} & \multirow[b]{2}{*}{71} \\
\hline TOTAL & & & & & & & & & \\
\hline
\end{tabular}

*Only those diagnostic categories and gender/age combinations with at least one OSHA event appear in this table. 


\section{Savannah River Site 2006}

OSHA Data

Appendix P. Number of Workdays Lost or with Restricted Activity in Each Diagnostic Category by Gender and Age*

\begin{tabular}{|c|c|c|c|c|c|}
\hline & & \multicolumn{4}{|c|}{ Women } \\
\hline & & \multicolumn{4}{|c|}{ Age Group } \\
\hline & & \multicolumn{2}{|c|}{$40-49$} & \multicolumn{2}{|c|}{$50+$} \\
\hline & & $\begin{array}{c}\text { Days } \\
\text { Restricted }\end{array}$ & Days Lost & $\begin{array}{c}\text { Days } \\
\text { Restricted }\end{array}$ & Days Lost \\
\hline Diagnostic Category & ICD-9-CM Codes & \multirow[b]{2}{*}{0} & \multirow[b]{2}{*}{0} & \multirow[b]{2}{*}{0} & \multirow[b]{2}{*}{0} \\
\hline -Chronic Obstructive Dis & $490-496$ & & & & \\
\hline -Lung Dis from External Agents & $500-508$ & 0 & 0 & 0 & 0 \\
\hline -Oral Cavity, Saliva Glands, Jaw & $520-529$ & 0 & 0 & 0 & 0 \\
\hline -Symptoms & 780-789 & 0 & 0 & 0 & 0 \\
\hline -Fracture - Neck, Trunk & $805-809$ & 0 & 0 & 0 & 0 \\
\hline -Sprains \& Strains - Other & $840-845,848$ & 0 & 0 & 33 & 0 \\
\hline -Open Wound - Head, Neck, Trunk & 870-879 & 0 & 0 & 0 & 0 \\
\hline -Open Wound - Upper Limb & 880-887 & 2 & 0 & 0 & 0 \\
\hline -Superficial Injury & $910-919$ & 0 & 0 & 0 & 0 \\
\hline -Contusion & $920-924$ & 0 & 0 & 0 & 0 \\
\hline $\begin{array}{l}\text {-Unspecified Effects - External } \\
\text { Causes }\end{array}$ & $990-995$ & 0 & 0 & 0 & 0 \\
\hline
\end{tabular}

\begin{tabular}{|c|c|c|c|c|c|c|c|c|c|}
\hline & & \multicolumn{8}{|c|}{ Men } \\
\hline & & \multicolumn{8}{|c|}{ Age Group } \\
\hline & & \multicolumn{2}{|c|}{$16-29$} & \multicolumn{2}{|c|}{$30-39$} & \multicolumn{2}{|c|}{$40-49$} & \multicolumn{2}{|c|}{$50+$} \\
\hline & & $\begin{array}{c}\text { Days } \\
\text { Restricted }\end{array}$ & $\begin{array}{l}\text { Days } \\
\text { Lost }\end{array}$ & $\begin{array}{c}\text { Days } \\
\text { Restricted }\end{array}$ & $\begin{array}{l}\text { Days } \\
\text { Lost }\end{array}$ & $\begin{array}{c}\text { Days } \\
\text { Restricted }\end{array}$ & $\begin{array}{l}\text { Days } \\
\text { Lost }\end{array}$ & $\begin{array}{c}\text { Days } \\
\text { Restricted }\end{array}$ & $\begin{array}{l}\text { Days } \\
\text { Lost }\end{array}$ \\
\hline Diagnostic Category & ICD-9-CM Codes & & & & & & & & \\
\hline -Disorders of Eye & $360-379$ & 0 & 0 & 0 & 0 & 0 & 0 & 0 & 0 \\
\hline -Chronic Obstructive Dis & $490-496$ & 0 & 0 & 0 & 0 & 0 & 0 & 0 & 0 \\
\hline -Lung Dis from External Agents & $500-508$ & 0 & 0 & 0 & 0 & 0 & 0 & 0 & 0 \\
\hline -Hernia & $550-553$ & 41 & 0 & 0 & 0 & 0 & 0 & 14 & 20 \\
\hline -Arthropathies & $710-719$ & 0 & 0 & 0 & 0 & 6 & 0 & 29 & 0 \\
\hline -Dorsopathies & $720-724$ & 0 & 0 & 0 & 0 & 0 & 0 & 29 & 0 \\
\hline -Rheumatism, Excluding Back & $725-729$ & 0 & 0 & 0 & 0 & 0 & 0 & 29 & 0 \\
\hline -Symptoms & $780-789$ & 0 & 0 & 0 & 0 & 0 & 0 & 0 & 0 \\
\hline -Fracture - Upper Limb & $810-819$ & 0 & 0 & 0 & 0 & 0 & 0 & 0 & 0 \\
\hline -Sprains \& Strains - Back & 846-847 & 0 & 0 & 0 & 0 & 56 & 0 & 0 & 0 \\
\hline -Sprains \& Strains - Other & $840-845,848$ & 0 & 0 & 0 & 0 & 0 & 0 & 161 & 19 \\
\hline -Open Wound - Head, Neck, Trunk & \begin{tabular}{|c|}
$870-879$ \\
\end{tabular} & 0 & 0 & 0 & 0 & 0 & 0 & 0 & 0 \\
\hline -Open Wound - Upper Limb & 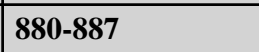 & 0 & 0 & 0 & 0 & 59 & 0 & 0 & 0 \\
\hline -Superficial Injury & $910-919$ & 0 & 0 & 0 & 0 & 0 & 0 & 0 & 7 \\
\hline -Contusion & $920-924$ & 0 & 0 & 0 & 0 & 0 & 0 & 0 & 0 \\
\hline
\end{tabular}

(Continued)

*OSHA events with >1 ICD-9-CM code in the same diagnostic category were counted only once. Only those diagnostic categories and gender/age combinations with at least one occurrence appear in this table. 
Savannah River Site 2006

OSHA Data

Appendix P. Number of Workdays Lost or with Restricted Activity in Each Diagnostic Category by Gender and Age*

\begin{tabular}{|c|c|c|c|c|c|c|c|c|c|}
\hline & & \multicolumn{8}{|c|}{ Men } \\
\hline & & \multicolumn{8}{|c|}{ Age Group } \\
\hline & & \multicolumn{2}{|c|}{$16-29$} & \multicolumn{2}{|c|}{30 - 39} & \multicolumn{2}{|c|}{$40-49$} & \multicolumn{2}{|c|}{$50+$} \\
\hline & & $\begin{array}{c}\text { Days } \\
\text { Restricted }\end{array}$ & $\begin{array}{l}\text { Days } \\
\text { Lost }\end{array}$ & $\begin{array}{c}\text { Days } \\
\text { Restricted }\end{array}$ & $\begin{array}{l}\text { Days } \\
\text { Lost }\end{array}$ & $\begin{array}{c}\text { Days } \\
\text { Restricted }\end{array}$ & $\begin{array}{l}\text { Days } \\
\text { Lost }\end{array}$ & $\begin{array}{c}\text { Days } \\
\text { Restricted }\end{array}$ & $\begin{array}{l}\text { Days } \\
\text { Lost }\end{array}$ \\
\hline Diagnostic Category & ICD-9-CM Codes & & & & & & & & \\
\hline -Foreign Body Entering Orifice & 930-939 & 0 & 0 & 0 & 0 & 0 & 0 & 0 & 0 \\
\hline -Burns & $940-949$ & 0 & 0 & 0 & 0 & 0 & 0 & 0 & 7 \\
\hline
\end{tabular}

*OSHA events with >1 ICD-9-CM code in the same diagnostic category were counted only once. Only those diagnostic categories and gender/age combinations with at least one occurrence appear in this table. 
Savannah River Site 2006

OSHA Data

Appendix Q. Number of Occurrences in Each Accident Category by Gender and Age*

\begin{tabular}{|c|c|c|c|c|c|c|c|c|c|c|}
\hline & & \multicolumn{3}{|c|}{ Women } & \multicolumn{5}{|c|}{ Men } & \multirow[b]{3}{*}{ TOTAL } \\
\hline & & \multicolumn{2}{|c|}{ Age Group } & \multirow[b]{2}{*}{ TOTAL } & \multicolumn{4}{|c|}{ Age Group } & \multirow[b]{2}{*}{ TOTAL } & \\
\hline & & $40-49$ & $50+$ & & $16-29$ & 30 - 39 & $40-49$ & $50+$ & & \\
\hline Motor Vehicle Traffic & E810-E819 & 0 & 0 & 0 & 0 & 0 & 0 & 1 & 1 & 1 \\
\hline Falls & E880-E888 & 1 & 2 & 3 & 0 & 0 & 1 & 3 & 4 & 7 \\
\hline Natural/Environmental Factors & E900-E909 & 1 & 0 & 1 & 0 & 0 & 0 & 0 & 0 & 1 \\
\hline $\begin{array}{l}\text { Submersion/Suffocation/Foreign } \\
\text { Bodies }\end{array}$ & E910-E915 & 0 & 0 & 0 & 0 & 0 & 1 & 1 & 2 & 2 \\
\hline Other Accidents & E916-E928 & 2 & 1 & 3 & 2 & 6 & 11 & 8 & 27 & 30 \\
\hline
\end{tabular}

*Only those accident types and gender/age combinations with at least one occurrence appear in this table. 
Savannah River Site 2006

OSHA Data

Appendix R. Number of Workdays Lost or with Restricted Activity in Each Accident Category by Gender and Age*

\begin{tabular}{|c|c|c|c|c|c|}
\hline & & \multicolumn{4}{|c|}{ Women } \\
\hline & & \multicolumn{4}{|c|}{ Age Group } \\
\hline & & \multicolumn{2}{|c|}{$40-49$} & \multicolumn{2}{|c|}{$50+$} \\
\hline & & $\begin{array}{c}\text { Days } \\
\text { Restricted }\end{array}$ & Days Lost & $\begin{array}{c}\text { Days } \\
\text { Restricted }\end{array}$ & Days Lost \\
\hline Type of Accident & E Codes & \multirow[b]{2}{*}{0} & \multirow[b]{2}{*}{0} & \multirow[b]{2}{*}{33} & \multirow[b]{2}{*}{0} \\
\hline Falls & E880-E888 & & & & \\
\hline Fire & E890-E899 & 0 & 0 & 0 & 0 \\
\hline Natural/Environmental Factors & E900-E909 & 0 & 0 & 0 & 0 \\
\hline Other Accidents & E916-E928 & 2 & 0 & 0 & 0 \\
\hline
\end{tabular}

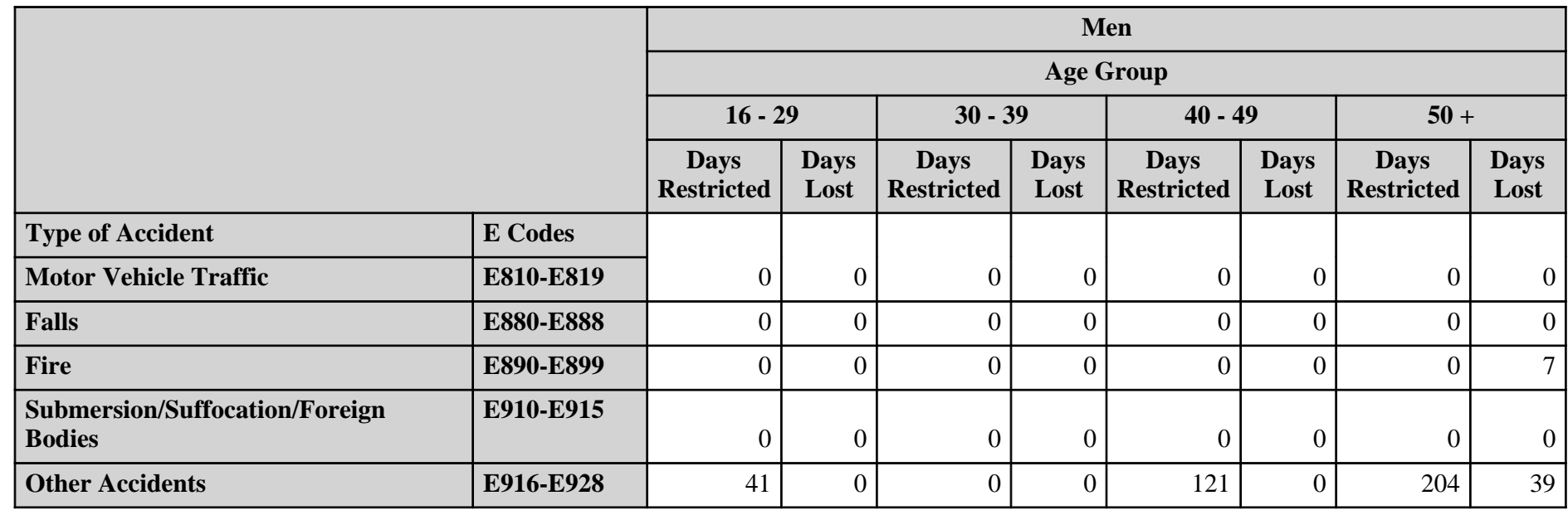

*OSHA events with $>1 \mathrm{E}$ code in the same accident type were counted only once. Only those accident types and gender/age combinations with at least one occurrence appear in this table. 


\section{Savannah River Site 2006}

\section{OSHA Data}

Appendix S. Number of Diagnoses in Each Diagnostic Category by Gender and Job Category*

\begin{tabular}{|c|c|c|c|c|c|}
\hline & & \multicolumn{4}{|c|}{ Women } \\
\hline & & \multicolumn{3}{|c|}{ Job Category } & \multirow[b]{2}{*}{ TOTAL } \\
\hline & & Professional & Technical Support & Line Operators & \\
\hline Diagnostic Category & ICD-9-CM Code & & & & \\
\hline $\begin{array}{l}\text { RESPIRATORY } \\
\text { SYSTEM }\end{array}$ & $460-519$ & 1 & 1 & 1 & 3 \\
\hline -Chronic Obstructive Dis & $490-496$ & 0 & 1 & 0 & 1 \\
\hline $\begin{array}{l}\text {-Lung Dis from External } \\
\text { Agents }\end{array}$ & $500-508$ & 1 & 0 & 1 & 2 \\
\hline DIGESTIVE SYSTEM & $520-579$ & 0 & 0 & 1 & 1 \\
\hline $\begin{array}{l}\text {-Oral Cavity, Saliva } \\
\text { Glands, Jaw }\end{array}$ & $520-529$ & 0 & 0 & 1 & 1 \\
\hline $\begin{array}{l}\text { SYMPTOMS, } \\
\text { SIGNS, \& ILL-DEFINED } \\
\text { CONDITIONS }\end{array}$ & $780-799$ & 2 & 0 & 1 & 3 \\
\hline -Symptoms & $780-789$ & 2 & 0 & 1 & 3 \\
\hline INJURY \& POISONING & $800-999$ & 3 & 2 & 5 & 10 \\
\hline -Fracture - Neck, Trunk & $805-809$ & 1 & 0 & 0 & 1 \\
\hline $\begin{array}{l}\text {-Sprains \& Strains - } \\
\text { Other }\end{array}$ & $840-845,848$ & 1 & 0 & 2 & 3 \\
\hline $\begin{array}{l}\text {-Open Wound - Head, } \\
\text { Neck, Trunk }\end{array}$ & 870-879 & 0 & 0 & 1 & 1 \\
\hline $\begin{array}{l}\text {-Open Wound - Upper } \\
\text { Limb }\end{array}$ & 880-887 & 0 & 1 & 0 & 1 \\
\hline -Superficial Injury & 910-919 & 0 & 0 & 1 & 1 \\
\hline -Contusion & $920-924$ & 1 & 0 & 1 & 2 \\
\hline $\begin{array}{l}\text {-Unspecified } \\
\text { Effects - External Causes }\end{array}$ & $990-995$ & 0 & 1 & 0 & 1 \\
\hline
\end{tabular}

\begin{tabular}{|c|c|c|c|c|}
\hline & \multicolumn{4}{|c|}{ Women } \\
\hline & \multicolumn{3}{|c|}{ Job Category } & \multirow[b]{2}{*}{ TOTAL } \\
\hline & Professional & Technical Support & Line Operators & \\
\hline Diagnostic Category & \multirow[b]{2}{*}{6} & \multirow[b]{2}{*}{3} & \multirow[b]{2}{*}{8} & \multirow[b]{2}{*}{17} \\
\hline TOTAL & & & & \\
\hline
\end{tabular}

*Only those diagnostic categories and gender/job category combinations with at least one occurrence appear in this table. 


\section{Savannah River Site 2006}

OSHA Data

Appendix S. Number of Diagnoses in Each Diagnostic Category by Gender and Job Category*

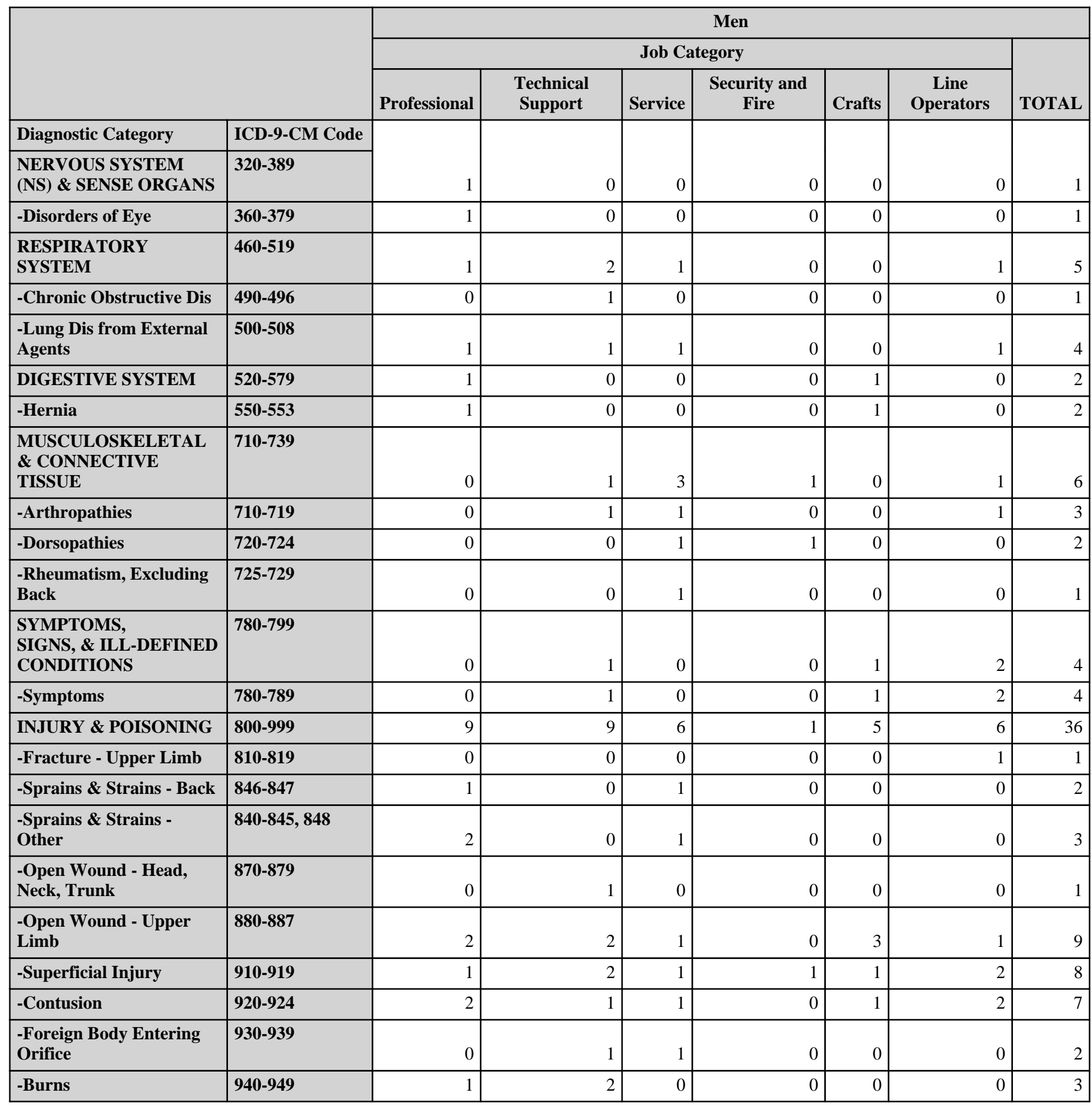

*Only those diagnostic categories and gender/job category combinations with at least one occurrence appear in this table. 
Savannah River Site 2006

OSHA Data

Appendix S. Number of Diagnoses in Each Diagnostic Category by Gender and Job Category*

\begin{tabular}{|c|c|c|c|c|c|c|c|}
\hline & \multicolumn{7}{|c|}{ Men } \\
\hline & \multicolumn{6}{|c|}{ Job Category } & \multirow[b]{2}{*}{ TOTAL } \\
\hline & Professional & Technical Support & Service & Security and Fire & Crafts & Line Operators & \\
\hline Diagnostic Category & \multirow[b]{2}{*}{12} & \multirow[b]{2}{*}{13} & \multirow[b]{2}{*}{10} & \multirow[b]{2}{*}{2} & \multirow[b]{2}{*}{7} & \multirow[b]{2}{*}{10} & \multirow[b]{2}{*}{54} \\
\hline TOTAL & & & & & & & \\
\hline
\end{tabular}

*Only those diagnostic categories and gender/job category combinations with at least one occurrence appear in this table. 


\section{Savannah River Site 2006}

OSHA Data

Appendix T. Number of Workdays Lost or with Restricted Activity in Each Diagnostic Category by Gender and Job Category*

\begin{tabular}{|c|c|c|c|c|c|c|c|}
\hline & & \multicolumn{6}{|c|}{ Women } \\
\hline & & \multicolumn{6}{|c|}{ Job Category } \\
\hline & & \multicolumn{2}{|c|}{ Professional } & \multicolumn{2}{|c|}{ Technical Support } & \multicolumn{2}{|c|}{ Line Operators } \\
\hline & & $\begin{array}{c}\text { Days } \\
\text { Restricted }\end{array}$ & Days Lost & \begin{tabular}{c|} 
Days \\
Restricted
\end{tabular} & Days Lost & $\begin{array}{c}\text { Days } \\
\text { Restricted }\end{array}$ & Days Lost \\
\hline Diagnostic Category & ICD-9-CM Codes & \multirow[b]{2}{*}{0} & \multirow[b]{2}{*}{0} & \multirow[b]{2}{*}{0} & \multirow[b]{2}{*}{0} & \multirow[b]{2}{*}{0} & \multirow[b]{2}{*}{0} \\
\hline -Chronic Obstructive Dis & $490-496$ & & & & & & \\
\hline -Lung Dis from External Agents & $500-508$ & 0 & 0 & 0 & 0 & 0 & 0 \\
\hline -Oral Cavity, Saliva Glands, Jaw & $520-529$ & 0 & 0 & 0 & 0 & 0 & 0 \\
\hline -Symptoms & 780-789 & 0 & 0 & 0 & 0 & 0 & 0 \\
\hline -Fracture - Neck, Trunk & 805-809 & 0 & 0 & 0 & 0 & 0 & 0 \\
\hline -Sprains \& Strains - Other & $840-845,848$ & 0 & 0 & 0 & 0 & 33 & 0 \\
\hline -Open Wound - Head, Neck, Trunk & $870-879$ & 0 & 0 & 0 & 0 & 0 & 0 \\
\hline -Open Wound - Upper Limb & $880-887$ & 0 & 0 & 2 & 0 & 0 & 0 \\
\hline -Superficial Injury & $910-919$ & 0 & 0 & 0 & 0 & 0 & 0 \\
\hline -Contusion & $920-924$ & 0 & 0 & 0 & 0 & 0 & 0 \\
\hline $\begin{array}{l}\text {-Unspecified Effects - External } \\
\text { Causes }\end{array}$ & $990-995$ & 0 & 0 & 0 & 0 & 0 & 0 \\
\hline
\end{tabular}

*OSHA events with >1 ICD-9-CM code in the same diagnostic category were counted only once. Only those diagnostic categories and gender/job category combinations with at least one occurrence appear in this table. 


\section{Savannah River Site 2006}

OSHA Data

Appendix T. Number of Workdays Lost or with Restricted Activity in Each Diagnostic Category by Gender and Job Category*

\begin{tabular}{|c|c|c|c|c|c|c|c|}
\hline & & \multicolumn{6}{|c|}{ Men } \\
\hline & & \multicolumn{6}{|c|}{ Job Category } \\
\hline & & \multicolumn{2}{|c|}{ Professional } & \multicolumn{2}{|c|}{ Technical Support } & \multicolumn{2}{|c|}{ Service } \\
\hline & & $\begin{array}{c}\text { Days } \\
\text { Restricted }\end{array}$ & Days Lost & $\begin{array}{c}\text { Days } \\
\text { Restricted }\end{array}$ & Days Lost & \begin{tabular}{|c|} 
Days \\
Restricted
\end{tabular} & Days Lost \\
\hline Diagnostic Category & ICD-9-CM Codes & \multirow[b]{2}{*}{0} & \multirow[b]{2}{*}{0} & \multirow[b]{2}{*}{0} & \multirow[b]{2}{*}{0} & \multirow[b]{2}{*}{0} & \multirow[b]{2}{*}{0} \\
\hline -Disorders of Eye & $360-379$ & & & & & & \\
\hline -Chronic Obstructive Dis & $490-496$ & 0 & 0 & 0 & 0 & 0 & 0 \\
\hline -Lung Dis from External Agents & $500-508$ & 0 & 0 & 0 & 0 & 0 & 0 \\
\hline -Hernia & $550-553$ & 14 & 20 & 0 & 0 & 0 & $\overline{0}$ \\
\hline -Arthropathies & $710-719$ & 0 & 0 & 0 & 0 & 29 & 0 \\
\hline -Dorsopathies & $720-724$ & 0 & 0 & 0 & 0 & 29 & 0 \\
\hline -Rheumatism, Excluding Back & $725-729$ & 0 & 0 & 0 & 0 & 29 & 0 \\
\hline -Symptoms & $780-789$ & 0 & 0 & 0 & 0 & 0 & 0 \\
\hline -Fracture - Upper Limb & 810-819 & 0 & 0 & 0 & 0 & 0 & $\overline{0}$ \\
\hline -Sprains \& Strains - Back & 846-847 & 0 & 0 & 0 & 0 & 56 & 0 \\
\hline -Sprains \& Strains - Other & $840-845,848$ & 0 & 0 & 0 & 0 & 161 & 19 \\
\hline -Open Wound - Head, Neck, Trunk & $870-879$ & 0 & 0 & 0 & 0 & 0 & $\overline{0}$ \\
\hline -Open Wound - Upper Limb & 880-887 & 0 & 0 & 0 & 0 & 0 & 0 \\
\hline -Superficial Injury & $910-919$ & 0 & 7 & 0 & 0 & 0 & 0 \\
\hline -Contusion & $920-924$ & 0 & 0 & 0 & 0 & 0 & 0 \\
\hline -Foreign Body Entering Orifice & $930-939$ & 0 & 0 & 0 & 0 & 0 & 0 \\
\hline -Burns & $940-949$ & 0 & 7 & 0 & 0 & 0 & 0 \\
\hline
\end{tabular}

*OSHA events with >1 ICD-9-CM code in the same diagnostic category were counted only once. Only those diagnostic categories and gender/job category combinations with at least one occurrence appear in this table. 


\section{Savannah River Site 2006}

OSHA Data

Appendix T. Number of Workdays Lost or with Restricted Activity in Each Diagnostic Category by Gender and Job Category*

\begin{tabular}{|c|c|c|c|c|c|c|c|}
\hline & & \multicolumn{6}{|c|}{ Men } \\
\hline & & \multicolumn{6}{|c|}{ Job Category } \\
\hline & & \multicolumn{2}{|c|}{ Security and Fire } & \multicolumn{2}{|c|}{ Crafts } & \multicolumn{2}{|c|}{ Line Operators } \\
\hline & & $\begin{array}{c}\text { Days } \\
\text { Restricted }\end{array}$ & Days Lost & \begin{tabular}{|c|} 
Days \\
Restricted
\end{tabular} & Days Lost & \begin{tabular}{|c|} 
Days \\
Restricted
\end{tabular} & Days Lost \\
\hline Diagnostic Category & ICD-9-CM Codes & \multirow[b]{2}{*}{0} & \multirow[b]{2}{*}{0} & \multirow[b]{2}{*}{0} & \multirow[b]{2}{*}{0} & \multirow[b]{2}{*}{0} & \multirow[b]{2}{*}{0} \\
\hline -Disorders of Eye & $360-379$ & & & & & & \\
\hline -Chronic Obstructive Dis & $490-496$ & 0 & 0 & 0 & 0 & 0 & 0 \\
\hline -Lung Dis from External Agents & $500-508$ & 0 & 0 & 0 & 0 & 0 & 0 \\
\hline -Hernia & $550-553$ & 0 & 0 & 41 & 0 & 0 & 0 \\
\hline -Arthropathies & $710-719$ & 0 & 0 & 0 & 0 & 6 & 0 \\
\hline -Dorsopathies & $720-724$ & 0 & 0 & 0 & 0 & 0 & 0 \\
\hline -Rheumatism, Excluding Back & $725-729$ & 0 & 0 & 0 & 0 & 0 & 0 \\
\hline -Symptoms & 780-789 & 0 & 0 & 0 & 0 & 0 & 0 \\
\hline -Fracture - Upper Limb & 810-819 & 0 & 0 & 0 & 0 & 0 & 0 \\
\hline -Sprains \& Strains - Back & $846-847$ & 0 & 0 & 0 & 0 & 0 & 0 \\
\hline -Sprains \& Strains - Other & $840-845,848$ & 0 & 0 & 0 & 0 & 0 & 0 \\
\hline -Open Wound - Head, Neck, Trunk & 870-879 & 0 & 0 & 0 & 0 & 0 & 0 \\
\hline -Open Wound - Upper Limb & 880-887 & 0 & 0 & 0 & 0 & 59 & 0 \\
\hline -Superficial Injury & $910-919$ & 0 & 0 & 0 & 0 & 0 & 0 \\
\hline -Contusion & $920-924$ & 0 & 0 & 0 & 0 & 0 & 0 \\
\hline -Foreign Body Entering Orifice & 930-939 & 0 & 0 & 0 & 0 & 0 & 0 \\
\hline -Burns & $940-949$ & 0 & 0 & 0 & 0 & 0 & 0 \\
\hline
\end{tabular}

*OSHA events with >1 ICD-9-CM code in the same diagnostic category were counted only once. Only those diagnostic categories and gender/job category combinations with at least one occurrence appear in this table. 


\section{Savannah River Site 2006}

OSHA Data

Appendix U. Number of Occurrences in Each Accident Category by Gender and Job Category*

\begin{tabular}{|c|c|c|c|c|c|}
\hline & & \multicolumn{4}{|c|}{ Women } \\
\hline & & \multicolumn{3}{|c|}{ Job Category } & \multirow[b]{2}{*}{ TOTAL } \\
\hline & & Professional & $\begin{array}{l}\text { Technical } \\
\text { Support }\end{array}$ & $\begin{array}{c}\text { Line } \\
\text { Operators }\end{array}$ & \\
\hline Type of Accident & E CODES & \multirow[b]{2}{*}{1} & \multirow[b]{2}{*}{0} & \multirow[b]{2}{*}{2} & \multirow[b]{2}{*}{3} \\
\hline Falls & E880-E888 & & & & \\
\hline Fire & E890-E899 & 1 & 0 & 1 & 2 \\
\hline Natural/Environmental Factors & E900-E909 & 0 & 1 & 0 & 1 \\
\hline Other Accidents & E916-E928 & 1 & 1 & 1 & 3 \\
\hline
\end{tabular}

\begin{tabular}{|c|c|c|c|c|c|c|c|c|}
\hline & & \multicolumn{7}{|c|}{ Men } \\
\hline & & \multicolumn{6}{|c|}{ Job Category } & \multirow[b]{2}{*}{ TOTAL } \\
\hline & & Professional & $\begin{array}{l}\text { Technical } \\
\text { Support }\end{array}$ & Service & \begin{tabular}{|} 
Security and \\
Fire
\end{tabular} & Crafts & $\begin{array}{c}\text { Line } \\
\text { Operators }\end{array}$ & \\
\hline Type of Accident & E CODES & \multirow[b]{2}{*}{0} & \multirow[b]{2}{*}{1} & \multirow[b]{2}{*}{0} & \multirow[b]{2}{*}{0} & \multirow[b]{2}{*}{0} & \multirow[b]{2}{*}{0} & \multirow[b]{2}{*}{1} \\
\hline Motor Vehicle Traffic & E810-E819 & & & & & & & \\
\hline Falls & E880-E888 & 0 & 0 & 0 & 1 & 1 & 2 & 4 \\
\hline Fire & E890-E899 & 1 & 1 & 0 & 0 & 0 & 1 & 3 \\
\hline $\begin{array}{l}\text { Submersion/Suffocation/Foreign } \\
\text { Bodies }\end{array}$ & E910-E915 & 0 & 1 & 1 & 0 & 0 & 0 & 2 \\
\hline Other Accidents & E916-E928 & 7 & 7 & 5 & 1 & 4 & 3 & 27 \\
\hline
\end{tabular}

*Only those accident types and gender/job category combinations with at least one occurrence appear in this table. 


\section{Savannah River Site 2006}

OSHA Data

Appendix V. Number of Workdays Lost or with Restricted Activity in Each Accident Category by Gender and Job Category*

\begin{tabular}{|c|c|c|c|c|c|c|c|}
\hline & & \multicolumn{6}{|c|}{ Women } \\
\hline & & \multicolumn{6}{|c|}{ Job Category } \\
\hline & & \multicolumn{2}{|c|}{ Professional } & \multicolumn{2}{|c|}{ Technical Support } & \multicolumn{2}{|c|}{ Line Operators } \\
\hline & & $\begin{array}{c}\text { Days } \\
\text { Restricted }\end{array}$ & Days Lost & $\begin{array}{c}\text { Days } \\
\text { Restricted }\end{array}$ & Days Lost & $\begin{array}{c}\text { Days } \\
\text { Restricted }\end{array}$ & Days Lost \\
\hline Type of Accident & E Codes & \multirow[b]{2}{*}{0} & \multirow[b]{2}{*}{0} & \multirow[b]{2}{*}{0} & \multirow[b]{2}{*}{0} & \multirow[b]{2}{*}{33} & \multirow[b]{2}{*}{0} \\
\hline Falls & E880-E888 & & & & & & \\
\hline Fire & E890-E899 & 0 & 0 & 0 & 0 & 0 & 0 \\
\hline Natural/Environmental Factors & E900-E909 & 0 & 0 & 0 & 0 & 0 & 0 \\
\hline Other Accidents & E916-E928 & 0 & 0 & 2 & 0 & 0 & 0 \\
\hline
\end{tabular}

\begin{tabular}{|c|c|c|c|c|c|c|c|}
\hline & \multicolumn{6}{|c|}{ Men } \\
\hline & & \multicolumn{6}{|c|}{ Job Category } \\
\hline & & \multicolumn{2}{|c|}{ Professional } & \multicolumn{2}{|c|}{ Technical Support } & \multicolumn{2}{|c|}{ Service } \\
\hline & & $\begin{array}{c}\text { Days } \\
\text { Restricted }\end{array}$ & Days Lost & $\begin{array}{c}\text { Days } \\
\text { Restricted }\end{array}$ & Days Lost & $\begin{array}{c}\text { Days } \\
\text { Restricted }\end{array}$ & Days Lost \\
\hline Type of Accident & E Codes & & & & & & \\
\hline Motor Vehicle Traffic & E810-E819 & 0 & 0 & 0 & 0 & 0 & 0 \\
\hline Falls & E880-E888 & 0 & 0 & 0 & 0 & 0 & 0 \\
\hline Fire & E890-E899 & 0 & 7 & 0 & 0 & 0 & 0 \\
\hline $\begin{array}{l}\text { Submersion/Suffocation/Foreign } \\
\text { Bodies }\end{array}$ & E910-E915 & 0 & 0 & 0 & 0 & 0 & 0 \\
\hline Other Accidents & E916-E928 & 14 & 20 & 0 & 0 & 246 & 19 \\
\hline
\end{tabular}

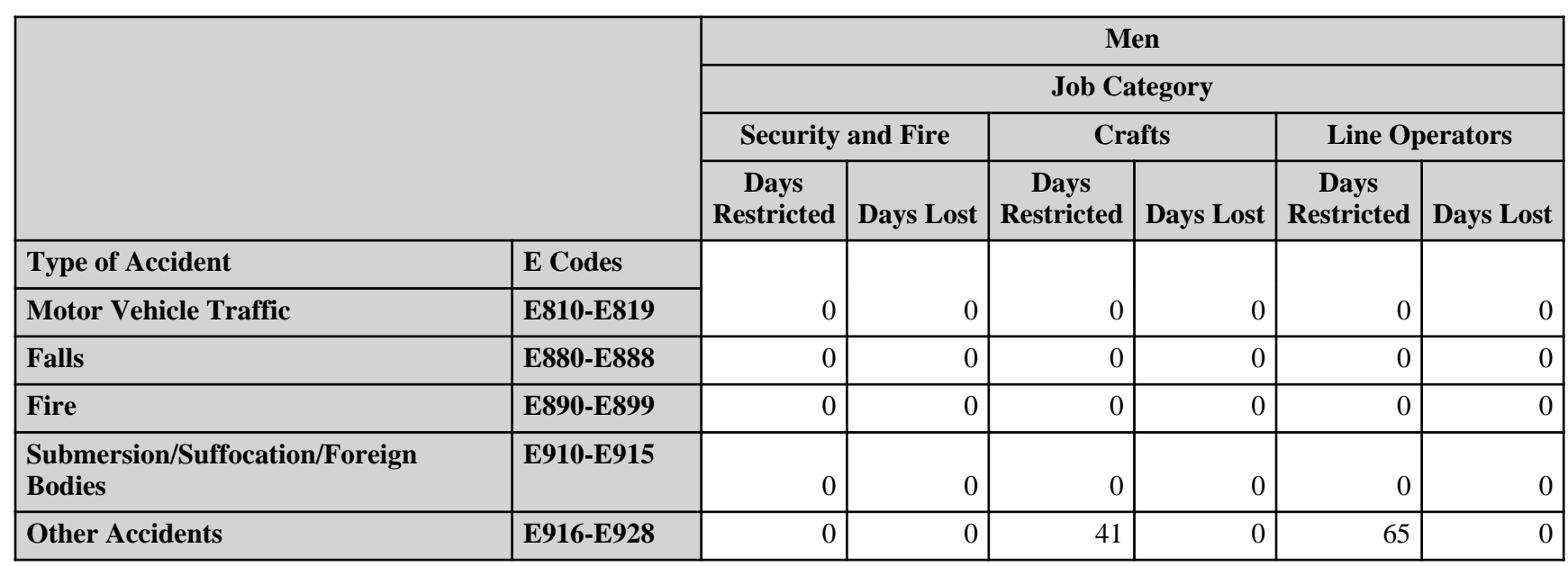

*OSHA events with $>1 \mathrm{E}$ code in the same accident type were counted only once. Only those accident types and gender/job category combinations with at least one occurrence appear in this table. 


\section{Savannah River Site 2006}

\section{OSHA Data}

Appendix W. Age-Adjusted OSHA Illness and Injury Rates by Diagnostic Category*

Part 1. Men

\begin{tabular}{|c|c|c|c|c|c|}
\hline & & $\begin{array}{l}\text { Number of } \\
\text { Diagnoses }\end{array}$ & $\begin{array}{c}\text { Age-Adjusted } \\
\text { Rate per } 1,000 * *\end{array}$ & $\begin{array}{c}\text { Lower } 95 \% \\
\text { Confidence } \\
\text { Limit per 1,000 }\end{array}$ & $\begin{array}{c}\text { Upper } 95 \% \\
\text { Confidence } \\
\text { Limit per 1,000 }\end{array}$ \\
\hline Diagnostic Category & ICD-9-CM Code & \multirow[b]{2}{*}{1} & \multirow[b]{2}{*}{0.1} & \multirow[b]{2}{*}{0.0} & \multirow[b]{2}{*}{0.4} \\
\hline NERVOUS SYSTEM (NS) \& SENSE ORGANS & 320-389 & & & & \\
\hline -Disorders of Eye & $360-379$ & 1 & 0.1 & 0.0 & 0.4 \\
\hline RESPIRATORY SYSTEM & $460-519$ & 5 & 0.3 & 0.1 & 0.7 \\
\hline -Chronic Obstructive Dis & $490-496$ & 1 & 0.1 & 0.0 & 0.4 \\
\hline -Lung Dis from External Agents & $500-508$ & 4 & 0.3 & 0.1 & 0.7 \\
\hline DIGESTIVE SYSTEM & $520-579$ & 2 & 1.4 & 0.2 & 9.2 \\
\hline -Hernia & $550-553$ & 2 & 1.4 & 0.2 & 9.2 \\
\hline $\begin{array}{l}\text { MUSCULOSKELETAL \& CONNECTIVE } \\
\text { TISSUE }\end{array}$ & 710-739 & 6 & 0.7 & 0.2 & 2.2 \\
\hline -Arthropathies & $710-719$ & 3 & 0.5 & 0.1 & 2.3 \\
\hline -Dorsopathies & $720-724$ & 2 & 0.1 & 0.0 & 0.6 \\
\hline -Rheumatism, Excluding Back & 725-729 & 1 & 0.1 & 0.0 & 0.4 \\
\hline $\begin{array}{l}\text { SYMPTOMS, SIGNS, \& ILL-DEFINED } \\
\text { CONDITIONS }\end{array}$ & $780-799$ & 4 & 0.3 & 0.1 & 0.7 \\
\hline -Symptoms & 780-789 & 4 & 0.3 & 0.1 & 0.7 \\
\hline INJURY \& POISONING & $800-999$ & 36 & 5.9 & 3.3 & 10.4 \\
\hline -Fracture - Upper Limb & 810-819 & 1 & 0.1 & 0.0 & 0.6 \\
\hline -Sprains \& Strains - Back & 846-847 & 2 & 0.5 & 0.1 & 2.4 \\
\hline -Sprains \& Strains - Other & $840-845,848$ & 3 & 1.5 & 0.2 & 8.8 \\
\hline -Open Wound - Head, Neck, Trunk & 870-879 & 1 & 0.1 & 0.0 & 0.6 \\
\hline -Open Wound - Upper Limb & 880-887 & 9 & 1.3 & 0.5 & 3.1 \\
\hline -Superficial Injury & 910-919 & 8 & 0.8 & 0.3 & 2.2 \\
\hline -Contusion & $920-924$ & 7 & 1.4 & 0.5 & 3.6 \\
\hline -Foreign Body Entering Orifice & 930-939 & 2 & 0.1 & 0.0 & 0.6 \\
\hline -Burns & $940-949$ & 3 & 0.2 & 0.1 & 0.5 \\
\hline Total & & 54 & 8.6 & 5.2 & 14.3 \\
\hline
\end{tabular}

Part 2. Women

\begin{tabular}{|c|c|c|c|c|c|}
\hline & & $\begin{array}{l}\text { Number of } \\
\text { Diagnoses }\end{array}$ & $\begin{array}{c}\text { Age-Adjusted } \\
\text { Rate per } 1,000^{* *}\end{array}$ & $\begin{array}{c}\text { Lower } 95 \% \\
\text { Confidence } \\
\text { Limit per 1,000 }\end{array}$ & $\begin{array}{c}\text { Upper } 95 \% \\
\text { Confidence } \\
\text { Limit per 1,000 }\end{array}$ \\
\hline Diagnostic Category & ICD-9-CM Code & \multirow[b]{2}{*}{3} & \multirow[b]{2}{*}{0.7} & \multirow[b]{2}{*}{0.2} & \multirow[b]{2}{*}{2.0} \\
\hline RESPIRATORY SYSTEM & $460-519$ & & & & \\
\hline -Chronic Obstructive Dis & $490-496$ & 1 & 0.2 & 0.0 & 1.5 \\
\hline -Lung Dis from External Agents & $500-508$ & 2 & 0.4 & 0.1 & 1.8 \\
\hline DIGESTIVE SYSTEM & $520-579$ & 1 & 0.2 & 0.0 & 1.5 \\
\hline
\end{tabular}

(Continued)

*Only those diagnostic categories with at least one occurrence appear in this table.

**Standardized to age distribution of 2000 U.S. population. 
Savannah River Site 2006

OSHA Data

Appendix W. Age-Adjusted OSHA Illness and Injury Rates by Diagnostic Category*

\begin{tabular}{|c|c|c|c|c|c|}
\hline & & $\begin{array}{l}\text { Number of } \\
\text { Diagnoses }\end{array}$ & $\begin{array}{c}\text { Age-Adjusted } \\
\text { Rate per } 1,000 * *\end{array}$ & $\begin{array}{c}\text { Lower } 95 \% \\
\text { Confidence } \\
\text { Limit per } 1,000\end{array}$ & $\begin{array}{c}\text { Upper } 95 \% \\
\text { Confidence } \\
\text { Limit per 1,000 }\end{array}$ \\
\hline Diagnostic Category & ICD-9-CM Code & \multirow[b]{2}{*}{1} & \multirow[b]{2}{*}{0.2} & \multirow[b]{2}{*}{0.0} & \multirow[b]{2}{*}{1.5} \\
\hline -Oral Cavity, Saliva Glands, Jaw & $520-529$ & & & & \\
\hline $\begin{array}{l}\text { SYMPTOMS, SIGNS, \& ILL-DEFINED } \\
\text { CONDITIONS }\end{array}$ & 780-799 & 3 & 0.7 & 0.2 & 2.0 \\
\hline -Symptoms & $780-789$ & 3 & 0.7 & 0.2 & 2.0 \\
\hline INJURY \& POISONING & 800-999 & 10 & 2.2 & 1.2 & 4.1 \\
\hline -Fracture - Skull & $800-804$ & 1 & 0.2 & 0.0 & 1.7 \\
\hline -Sprains \& Strains - Other & $840-845,848$ & 3 & 0.7 & 0.2 & 2.0 \\
\hline -Open Wound - Head, Neck, Trunk & $870-879$ & 1 & 0.2 & 0.0 & 1.5 \\
\hline -Open Wound - Upper Limb & 880-887 & 1 & 0.2 & 0.0 & 1.7 \\
\hline -Superficial Injury & $910-919$ & 1 & 0.2 & 0.0 & 1.5 \\
\hline -Contusion & $920-924$ & 2 & 0.4 & 0.1 & 1.7 \\
\hline -Toxic Effects - Non-medicinal & $980-989$ & 1 & 0.2 & 0.0 & 1.7 \\
\hline Total & & 17 & 3.7 & 2.3 & 6.0 \\
\hline
\end{tabular}

* Only those diagnostic categories with at least one occurrence appear in this table.

**Standardized to age distribution of 2000 U.S. population. 


\section{Savannah River Site 2006}

\section{OSHA Data}

Appendix W. Age-Adjusted OSHA Illness and Injury Rates by Diagnostic Category*

Part 3. Men and Women

\begin{tabular}{|c|c|c|c|c|c|}
\hline & & $\begin{array}{l}\text { Number of } \\
\text { Diagnoses }\end{array}$ & $\begin{array}{c}\text { Age-Adjusted } \\
\text { Rate per } 1,000 * *\end{array}$ & $\begin{array}{c}\text { Lower } 95 \% \\
\text { Confidence } \\
\text { Limit per 1,000 }\end{array}$ & $\begin{array}{c}\text { Upper } 95 \% \\
\text { Confidence } \\
\text { Limit per 1,000 }\end{array}$ \\
\hline Diagnostic Category & ICD-9-CM Code & \multirow[b]{2}{*}{1} & \multirow[b]{2}{*}{0.0} & \multirow[b]{2}{*}{0.0} & \multirow[b]{2}{*}{0.3} \\
\hline NERVOUS SYSTEM (NS) \& SENSE ORGANS & $320-389$ & & & & \\
\hline -Disorders of Eye & $360-379$ & 1 & 0.0 & 0.0 & 0.3 \\
\hline RESPIRATORY SYSTEM & $460-519$ & 8 & 0.4 & 0.2 & $\overline{0.8}$ \\
\hline -Chronic Obstructive Dis & $490-496$ & 2 & 0.1 & 0.0 & 0.4 \\
\hline -Lung Dis from External Agents & $500-508$ & 6 & 0.3 & 0.1 & 0.7 \\
\hline DIGESTIVE SYSTEM & $520-579$ & 3 & 1.1 & 0.2 & 6.7 \\
\hline -Oral Cavity, Saliva Glands, Jaw & $520-529$ & 1 & 0.0 & 0.0 & 0.3 \\
\hline -Hernia & $550-553$ & 2 & 1.1 & 0.2 & 6.9 \\
\hline $\begin{array}{l}\text { MUSCULOSKELETAL \& CONNECTIVE } \\
\text { TISSUE }\end{array}$ & \begin{tabular}{|l|}
$710-739$ \\
\end{tabular} & 6 & 0.5 & 0.2 & 1.6 \\
\hline -Arthropathies & $710-719$ & 3 & 0.4 & 0.1 & 1.7 \\
\hline -Dorsopathies & $720-724$ & 2 & 0.1 & 0.0 & 0.4 \\
\hline -Rheumatism, Excluding Back & $725-729$ & 1 & 0.0 & 0.0 & 0.3 \\
\hline $\begin{array}{l}\text { SYMPTOMS, SIGNS, \& ILL-DEFINED } \\
\text { CONDITIONS }\end{array}$ & 780-799 & 7 & 0.3 & 0.2 & 0.7 \\
\hline -Symptoms & $780-789$ & 7 & 0.3 & 0.2 & 0.7 \\
\hline INJURY \& POISONING & $800-999$ & 46 & 4.9 & 2.9 & 8.2 \\
\hline -Fracture - Skull & $800-804$ & 1 & 0.1 & 0.0 & 0.4 \\
\hline -Fracture - Upper Limb & 810-819 & 1 & 0.1 & 0.0 & 0.4 \\
\hline -Sprains \& Strains - Back & 846-847 & 2 & 0.3 & 0.1 & 1.8 \\
\hline -Sprains \& Strains - Other & $840-845,848$ & 6 & 1.3 & 0.3 & 6.1 \\
\hline -Open Wound - Head, Neck, Trunk & 870-879 & 2 & 0.1 & 0.0 & 0.4 \\
\hline -Open Wound - Upper Limb & $880-887$ & 10 & 1.0 & 0.4 & 2.3 \\
\hline -Superficial Injury & 910-919 & 9 & 0.7 & 0.3 & 1.7 \\
\hline -Contusion & $920-924$ & 9 & 1.1 & 0.5 & 2.7 \\
\hline -Foreign Body Entering Orifice & 930-939 & 2 & 0.1 & 0.0 & 0.4 \\
\hline -Burns & $940-949$ & 3 & 0.1 & 0.0 & 0.4 \\
\hline -Toxic Effects - Non-medicinal & $980-989$ & 1 & 0.1 & 0.0 & 0.4 \\
\hline Total & & 71 & 7.3 & 4.7 & 11.5 \\
\hline
\end{tabular}

* Only those diagnostic categories with at least one occurrence appear in this table.

**Standardized to age distribution of 2000 U.S. population. 